Japan. J. Math.

Vol. 29, No. 2, 2003

\title{
A note on extensions of algebraic and formal groups, $\mathrm{V}$
}

\author{
By Tsutomu Sekiguchi*) and Noriyuki Suwa**)
}

(Received October 22, 2002)

(from Tokyo Journal of Mathematics)

\begin{abstract}
We give an explicit description of homomorphisms and extensions of group schemes and formal groups, related to deformations connecting the multiplicative group and the additive group.
\end{abstract}

\section{Introduction}

Throughout the article, $p$ denotes a prime number. Let $W$ (resp. $\widehat{W}$ ) denote the group scheme (resp. the formal group scheme) of Witt vectors over $\mathbb{Z}$, and let $\mathbb{G}_{m}$ (resp. $\widehat{\mathbb{G}}_{m}$ ) denote the multiplicative group scheme (resp. the multiplicative formal group scheme) over $\mathbb{Z}$. Let $F$ be the Frobenius endomorphism of $W$ or of $\widehat{W}$ (for the definition see 1.2 ).

In the previous paper [13], we gave an explicit description of the groups $\operatorname{Ext}_{A}\left(\mathcal{G}^{(\lambda)}, \mathbb{G}_{m, A}\right)$ and $\operatorname{Ext}_{A}\left(\widehat{\mathcal{G}}^{(\lambda)}, \widehat{\mathbb{G}}_{m, A}\right)$ when $A$ is a $\mathbb{Z}_{(p)}$-algebra. Here $\lambda \in A$ and $\mathcal{G}^{(\lambda)}=\operatorname{Spec} A[T, 1 /(1+\lambda T)]$ is a group scheme giving a deformation of $\mathbb{G}_{a}$ to $\mathbb{G}_{m}$ (for the definition, see 3.1). More precisely, we constructed isomorphisms

$$
\begin{aligned}
\operatorname{Ker}\left[F-\left[\lambda^{p-1}\right]: W(A)\right. & \rightarrow W(A)] \stackrel{\sim}{\rightarrow} \operatorname{Hom}\left(\widehat{\mathcal{G}}^{(\lambda)}, \widehat{\mathbb{G}}_{m, A}\right), \\
\operatorname{Coker}\left[F-\left[\lambda^{p-1}\right]: W(A)\right. & \rightarrow W(A)] \stackrel{\sim}{\rightarrow} H_{0}^{2}\left(\widehat{\mathcal{G}}^{(\lambda)}, \widehat{\mathbb{G}}_{m, A}\right),
\end{aligned}
$$

using deformations of the Artin-Hasse exponential series. If $\lambda$ is nilpotent, we have also isomorphisms

$$
\begin{aligned}
\operatorname{Ker}\left[F-\left[\lambda^{p-1}\right]: \widehat{W}(A)\right. & \rightarrow \widehat{W}(A)] \stackrel{\sim}{\rightarrow} \operatorname{Hom}\left(\mathcal{G}^{(\lambda)}, \mathbb{G}_{m, A}\right), \\
\operatorname{Coker}\left[F-\left[\lambda^{p-1}\right]: \widehat{W}(A)\right. & \rightarrow \widehat{W}(A)] \stackrel{\sim}{\rightarrow} H_{0}^{2}\left(\mathcal{G}^{(\lambda)}, \mathbb{G}_{m, A}\right)
\end{aligned}
$$

Our aim of this article is to generalize the isomorphisms to that for $\widehat{\mathcal{G}}^{(\mu)}$ instead of $\widehat{\mathbb{G}}_{m, A}$. Precisely, our result is as follows.

1991 Mathematics Subject Classification. Primary 14L05; Secondary 13K05, 20G10.

*) Partially supported by Grant-in-Aid for Scientific Research No. 11640045.

**) Partially supported by Grant-in-Aid for Scientific Research No. 12640041. 
Theorem. Let $A$ be $a \mathbb{Z}_{(p)}$-algebra and $\lambda, \mu \in A$. Then there exist isomorphisms:

$$
\begin{aligned}
\operatorname{Ker}\left[F^{(\mu)}-\left[\lambda^{p-1}\right]: W^{(\mu)}(A)\right. & \left.\rightarrow W^{(\mu)}(A)\right] \stackrel{\sim}{\rightarrow} \operatorname{Hom}\left(\widehat{\mathcal{G}}^{(\lambda)}, \widehat{\mathcal{G}}^{(\mu)}\right), \\
\operatorname{Coker}\left[F^{(\mu)}-\left[\lambda^{p-1}\right]: W^{(\mu)}(A)\right. & \left.\rightarrow W^{(\mu)}(A)\right] \stackrel{\sim}{\rightarrow} H_{0}^{2}\left(\widehat{\mathcal{G}}^{(\lambda)}, \widehat{\mathcal{G}}^{(\mu)}\right) .
\end{aligned}
$$

Moreover, if $\lambda$ is nilpotent, there exist isomorphism:

$$
\begin{aligned}
\operatorname{Ker}\left[F^{(\mu)}-\left[\lambda^{p-1}\right]: \widehat{W}^{(\mu)}(A)\right. & \left.\rightarrow \widehat{W}^{(\mu)}(A)\right] \stackrel{\sim}{\rightarrow} \operatorname{Hom}\left(\mathcal{G}^{(\lambda)}, \mathcal{G}^{(\mu)}\right), \\
\operatorname{Coker}\left[F^{(\mu)}-\left[\lambda^{p-1}\right]: \widehat{W}^{(\mu)}(A)\right. & \left.\rightarrow \widehat{W}^{(\mu)}(A)\right] \stackrel{\sim}{\rightarrow} H_{0}^{2}\left(\mathcal{G}^{(\lambda)}, \mathcal{G}^{(\mu)}\right) .
\end{aligned}
$$

(Theorem 3.5.1 and Remark 3.12. For the definition of $W^{(\mu)}(A)$ and $F^{(\mu)}$, see the Section 1.)

It is crucial to define variants of Witt vectors and to construct deformations of the Artin-Hasse exponential series for an explicit description of the isomorphisms.

Now we explain the contents of the article.

The first two sections are devoted to prepare necessary tools to state the main theorem. In the Section 1 , we define variants of Witt vectors $W^{(M)}(A)$ for a $\mathbb{Z}[M]$-algebra $A$, paraphrasing the classical theory of Witt vectors. $W^{(M)}(A)$ is interpreted as the $A$-valued points of a group scheme $W^{(M)}$ over $\mathbb{Z}[M]$. At the end of the section, we construct an exact sequence of group schemes over $\mathbb{Z}[M]$

$$
0 \rightarrow W^{(M)} \rightarrow \prod_{B / A} W_{B} \rightarrow W_{A} \rightarrow 0
$$

where $A=\mathbb{Z}[M]$ and $B=A[t] /\left(t^{2}-M t\right)$.

In the Section 2, we define variants of the Artin-Hasse exponential series

$$
E_{p}^{(M)}(\boldsymbol{U}, \Lambda ; T) \in \mathbb{Z}_{(p)}\left[U_{0}, U_{1}, U_{2}, \ldots, \Lambda, M\right][[T]],
$$

modifying the power series

$$
E_{p}(\boldsymbol{U}, \Lambda ; T) \in \mathbb{Z}_{(p)}\left[U_{0}, U_{1}, U_{2}, \ldots, \Lambda\right][[T]]
$$

presented in [13]. The definition of $E_{p}^{(M)}(\boldsymbol{U}, \Lambda ; T)$ is parallel to that of $W^{(M)}$ in a sense. It would be worthy of note that we have a formula

$$
E_{p}^{\left(\Lambda^{p}\right)}\left(p[1]_{\Lambda}, \Lambda ; T\right)=\frac{(1+\Lambda T)^{p}-1}{\Lambda^{p}}
$$


(Example 3.9) because $\left\{(1+\Lambda T)^{p}-1\right\} / \Lambda^{p}$ defines the isogeny $\Psi$ in the KummerArtin-Schreier theory:

$$
0 \rightarrow \mathbb{Z} / p \mathbb{Z} \rightarrow \mathcal{G}^{(\lambda)} \stackrel{\Psi}{\rightarrow} \mathcal{G}^{\left(\lambda^{p}\right)} \rightarrow 0
$$

(cf. [15]).

In the Section 3, we prove the main result, after reviewing the Hochschild cohomology in our case. The theorem can be reduced to the main result of [13] thanks to an exact sequence of formal groups

$$
0 \rightarrow \widehat{\mathcal{G}}_{A}^{(M)} \rightarrow\left(\prod_{B / A} \widehat{\mathbb{G}}_{m, B}\right) \rightarrow \widehat{\mathbb{G}}_{m, A} \rightarrow 0
$$

where $A$ is a $\mathbb{Z}[M]$-algebra and $B=A[t] /\left(t^{2}-M t\right)$.

The last section is devoted to verify a compatibility of the main theorem with the Cartier theory on $p$-typical curves of formal groups. After a short review on the Dieudonné-Cartier algebra $D_{A}$ and the Cartier module of $p$-typical curves, we prove that $W^{(M)}(A)$ is isomorphic to the Cartier module of the formal group $\widehat{\mathcal{G}}^{(M)}$, giving an explicit description of $p$-typical curves of $\widehat{\mathcal{G}}^{(M)}$ (Theorem 4.15.1 and Proposition 4.19). It would be remarkable that the $D_{A}$-module $W^{(M)}(A)$ has a free resolution, written in a simple form (Theorem 4.4). We conclude the article, interpreting the main result mentioned above in the framework of the Cartier theory (Theorem 4.24.1 and Theorem 4.40.1). We have to introduce a formal power series

$$
G_{p}^{(\Lambda)}(\boldsymbol{Z}, \boldsymbol{U} ; \boldsymbol{T}) \in \mathbb{Z}_{(p)}\left[\Lambda, U_{0}, U_{1}, U_{2}, \ldots, Z_{0}, Z_{1}, Z_{2}, \ldots\right]\left[\left[T_{0}, T_{1}, T_{2}, \ldots\right]\right]
$$

to proceed with the argument for Theorem 4.40.1.

In this article we have established formalisms necessary to describe systematically the theory unifying the Kummer and Artin-Schreier-Witt theories, which permits us to explain a little more pleasantly the argument developed in [14]. Detailed accounts will appear elsewhere as a sequel to this work.

The authors express their hearty thanks to Michel Matignon, who provided us an excellent and inspiring environment for research. This work started with a conversation under la Porte d'Aquitaine at Bordeaux. They are very grateful also to Barry Green for his warm hospitality. The last part of the article was discussed on Helderberg near to Stellenbosch.

\section{Notation}

Throughout the paper, $p$ denotes a prime integer, $\mathbb{Z}_{(p)}$ the localization of $\mathbb{Z}$ at the prime ideal $(p)$, and $A$ a $\mathbb{Z}_{(p)}$-algebra. All rings are commutative with a unit element 1 , unless otherwise stated. 
$\mathbb{G}_{a, A}:$ the additive group scheme over $A$

$\mathbb{G}_{m, A}:$ the multiplicative group scheme over $A$

$W_{n, A}$ : the group scheme of Witt vectors of length $n$ over $A$

$W_{A}$ : the group scheme of Witt vectors over $A$

$\widehat{\mathbb{G}}_{a, A}:$ the additive formal group scheme over $A$

$\widehat{\mathbb{G}}_{m, A}$ : the multiplicative formal group scheme over $A$

$\widehat{W}_{n, A}$ : the formal group scheme of Witt vectors of length $n$ over $A$

$\widehat{W}_{A}$ : the formal group scheme of Witt vectors over $A$

$F:$ the Frobenius endomorphism of $W_{A}$

$V:$ the Verschiebung endomorphism of $W_{A}$

$[a]$ : the Teichmüller lifting $(a, 0,0, \ldots) \in W(A)$ of $a \in A$

$H_{0}^{2}(G, H)$ denotes the Hochschild cohomology group consisting of symmetric 2-cocycles of $G$ with coefficients in $H$ for group schemes or formal group schemes $G$ and $H$.

For a commutative ring $B, B^{\times}$denotes the multiplicative group $\mathbb{G}_{m}(B)$.

\section{List of formal power series}

$E_{p}(T):$ the Artin-Hasse exponential series, recalled in 2.1

$E_{p}(U, \Lambda ; T):$ recalled in 2.2

$E_{p}(\boldsymbol{U}, \Lambda ; T):$ recalled in 2.2

$E_{p}^{(M)}(U, \Lambda ; T):$ defined in 2.3

$F_{p}(\boldsymbol{U}, \Lambda ; X, Y):$ recalled in 2.5

$F_{p}^{(M)}(\boldsymbol{U}, \Lambda ; X, Y):$ defined in 2.7

$E_{p}(\boldsymbol{U} ; \boldsymbol{T}):$ recalled in 4.14

$E_{p}^{(M)}(\boldsymbol{U} ; \boldsymbol{T}):$ defined in 4.17

$G_{p}^{(\Lambda)}(\boldsymbol{Z}, \boldsymbol{U} ; \boldsymbol{T}): \quad$ defined in 4.29

$E_{p}^{(\Lambda, M)}(\boldsymbol{Z}, \boldsymbol{U}, \boldsymbol{V} ; \boldsymbol{T}):$ defined in 4.35

\section{Contents}

1. Around Witt vectors

2. Around the Artin-Hasse exponential series

3. Statement and proof of the theorem

4. Around the Cartier theory 


\section{Around Witt vectors}

We start with reviewing necessary facts on Witt vectors. For details, see [2, Chap. V] or [4, Chap. III].

1.1. For each $r \geq 0$, we denote by $\Phi_{r}(\boldsymbol{T})=\Phi_{r}\left(T_{0}, T_{1}, \ldots, T_{r}\right)$ the so-called Witt polynomial

$$
\Phi_{r}(\boldsymbol{T})=T_{0}^{p^{r}}+p T_{1}^{p^{r-1}}+\cdots+p^{r} T_{r}
$$

in $\mathbb{Z}[\boldsymbol{T}]=\mathbb{Z}\left[T_{0}, T_{1}, \ldots\right]$. We define polynomials

$$
S_{r}(\boldsymbol{X}, \boldsymbol{Y})=S_{r}\left(X_{0}, \ldots, X_{r}, Y_{0}, \ldots, Y_{r}\right)
$$

and

$$
P_{r}(\boldsymbol{X}, \boldsymbol{Y})=P_{r}\left(X_{0}, \ldots, X_{r}, Y_{0}, \ldots, Y_{r}\right)
$$

in $\mathbb{Z}[\boldsymbol{X}, \boldsymbol{Y}]=\mathbb{Z}\left[X_{0}, X_{1}, \ldots, Y_{0}, Y_{1}, \ldots\right]$ inductively by

$$
\Phi_{r}\left(S_{0}(\boldsymbol{X}, \boldsymbol{Y}), S_{1}(\boldsymbol{X}, \boldsymbol{Y}), \ldots, S_{r}(\boldsymbol{X}, \boldsymbol{Y})\right)=\Phi_{r}(\boldsymbol{X})+\Phi_{r}(\boldsymbol{Y})
$$

and

$$
\Phi_{r}\left(P_{0}(\boldsymbol{X}, \boldsymbol{Y}), P_{1}(\boldsymbol{X}, \boldsymbol{Y}), \ldots, P_{r}(\boldsymbol{X}, \boldsymbol{Y})\right)=\Phi_{r}(\boldsymbol{X}) \Phi_{r}(\boldsymbol{Y})
$$

Put

$$
\begin{aligned}
& S(\boldsymbol{X}, \boldsymbol{Y})=\left(S_{0}(\boldsymbol{X}, \boldsymbol{Y}), S_{1}(\boldsymbol{X}, \boldsymbol{Y}), S_{2}(\boldsymbol{X}, \boldsymbol{Y}), \ldots\right) \\
& P(\boldsymbol{X}, \boldsymbol{Y})=\left(P_{0}(\boldsymbol{X}, \boldsymbol{Y}), P_{1}(\boldsymbol{X}, \boldsymbol{Y}), P_{2}(\boldsymbol{X}, \boldsymbol{Y}), \ldots\right)
\end{aligned}
$$

Then as is well-known, the ring structure of the scheme of Witt vectors

$$
W_{\mathbb{Z}}=\operatorname{Spec} \mathbb{Z}\left[T_{0}, T_{1}, T_{2}, \ldots\right]
$$

is given by the addition

$$
\begin{aligned}
\boldsymbol{T} & =\left(T_{0}, T_{1}, T_{2}, \ldots\right) \mapsto S(\boldsymbol{T} \otimes 1,1 \otimes \boldsymbol{T}) \\
& =\left(S_{0}(\boldsymbol{T} \otimes 1,1 \otimes \boldsymbol{T}), S_{1}(\boldsymbol{T} \otimes 1,1 \otimes \boldsymbol{T}), S_{2}(\boldsymbol{T} \otimes 1,1 \otimes \boldsymbol{T}), \ldots\right)
\end{aligned}
$$

and the multiplication

$$
\begin{aligned}
\boldsymbol{T} & =\left(T_{0}, T_{1}, T_{2}, \ldots\right) \mapsto P(\boldsymbol{T} \otimes 1,1 \otimes \boldsymbol{T}) \\
& =\left(P_{0}(\boldsymbol{T} \otimes 1,1 \otimes \boldsymbol{T}), P_{1}(\boldsymbol{T} \otimes 1,1 \otimes \boldsymbol{T}), P_{2}(\boldsymbol{T} \otimes 1,1 \otimes \boldsymbol{T}), \ldots\right) .
\end{aligned}
$$

Define a morphism of schemes

$$
\Phi: W=\operatorname{Spec} \mathbb{Z}\left[T_{0}, T_{1}, T_{2}, \ldots\right] \rightarrow \mathbb{A}^{\mathbb{N}}=\operatorname{Spec} \mathbb{Z}\left[\Phi_{0}, \Phi_{1}, \Phi_{2}, \ldots\right]
$$


by

$$
\left(\Phi_{0}, \Phi_{1}, \Phi_{2}, \ldots\right) \mapsto\left(\Phi_{0}(T), \Phi_{1}(T), \Phi_{2}(T), \ldots\right) .
$$

Then $\Phi$ is a homomorphism of ring schemes. Let $A$ be a ring. If $p$ is invertible in $A, \Phi: W(A) \rightarrow A^{\mathbb{N}}$ is bijective. Hence, if the homothety by $p$ is injective on $A, \Phi: W(A) \rightarrow A^{\mathbb{N}}$ is injective. For $\boldsymbol{a} \in W(A), \Phi(\boldsymbol{a})=\left(\Phi_{0}(\boldsymbol{a}), \Phi_{1}(\boldsymbol{a}), \Phi_{2}(\boldsymbol{a}), \ldots\right)$ is called the phantom of $\boldsymbol{a}$.

We denote by $\widehat{W}_{\mathbb{Z}}$ the formal completion of $W_{\mathbb{Z}}$ along the zero section. $\widehat{W}_{\mathbb{Z}}$ is considered as a subfunctor of $W_{\mathbb{Z}}$. Indeed, if $A$ is a ring,

$$
\widehat{W}(A)=\left\{\left(a_{0}, a_{1}, a_{2}, \ldots\right) \in W_{n}(A) ; \begin{array}{l}
a_{i} \text { is nilpotent for all } i \text { and } \\
a_{i}=0 \text { for all but a finite number of } i
\end{array}\right\} .
$$

1.2. The Verschiebung homomorphism $V: W_{\mathbb{Z}} \rightarrow W_{\mathbb{Z}}$ is defined by

$$
T_{0} \mapsto 0, T_{1} \mapsto T_{0}, T_{2} \mapsto T_{1}, \ldots: \mathbb{Z}\left[T_{0}, T_{1}, T_{2}, \ldots\right] \rightarrow \mathbb{Z}\left[T_{0}, T_{1}, T_{2}, \ldots\right]
$$

Define now polynomials

$$
F_{r}(\boldsymbol{T})=F_{r}\left(T_{0}, \ldots, T_{r}, T_{r+1}\right) \in \mathbb{Q}\left[T_{0}, \ldots, T_{r}, T_{r+1}\right]
$$

inductively by

$$
\Phi_{r}\left(F_{0}(\boldsymbol{T}), \ldots, F_{r}(\boldsymbol{T})\right)=\Phi_{r+1}\left(T_{0}, \ldots, T_{r}, T_{r+1}\right)
$$

for $r \geq 0$. Then

$$
F_{r}(\boldsymbol{T})=F_{r}\left(T_{0}, \ldots, T_{r}, T_{r+1}\right) \in \mathbb{Z}\left[T_{0}, \ldots, T_{r}, T_{r+1}\right]
$$

for each $r \geq 0$. We denote by $F: W_{\mathbb{Z}} \rightarrow W_{\mathbb{Z}}$ the morphism defined by

$$
T_{0} \mapsto F_{0}(\boldsymbol{T}), T_{1} \mapsto F_{1}(\boldsymbol{T}), T_{2} \mapsto F_{2}(\boldsymbol{T}), \ldots: \mathbb{Z}\left[T_{0}, T_{1}, T_{2}, \ldots\right] \rightarrow \mathbb{Z}\left[T_{0}, T_{1}, T_{2}, \ldots\right] .
$$

Then it is verified without difficulty that $F$ is a homomorphism of ring schemes. It is obvious that $\widehat{W}_{\mathbb{Z}}$ is stable under $F$ (cf. [1, Ch. 9.1.3] or [3, Ch. O.1.3]). It is readily seen that $F_{r}(\boldsymbol{T}) \equiv T_{r}^{p} \bmod p$ for each $r \geq 0$. Therefore, if $A$ is an $\mathbb{F}_{p}$-algebra, $F: W_{A} \rightarrow W_{A}$ is nothing but the usual Frobenius endomorphism.

Let $A$ be a ring. Then we can verify without difficulty that:

(1) $F V=p$;

(2) $V(F(\boldsymbol{a}) \boldsymbol{b})=\boldsymbol{a} V(\boldsymbol{b})$ for $\boldsymbol{a}, \boldsymbol{b} \in W(A)$.

1.3. Let $A$ be a ring and $a \in A$. We denote the Witt vector $(a, 0,0, \ldots)$ with components in $A$ by $[a]$. $[a]$ is called the Teichmüller lifting of $a$. It is readily seen:

(1) $[a][b]=[a b]$;

(2) $F[a]=\left[a^{p}\right]$

(3) $\left(a_{0}, a_{1}, a_{2}, \ldots\right)=\sum_{k=0}^{\infty} V^{k}\left[a_{k}\right]$. 
1.4. Now we introduce variants of the Witt calculus. Define

$$
\Phi_{r}^{(M)}(\boldsymbol{T})=\Phi_{r}^{(M)}\left(T_{0}, \ldots, T_{r}\right) \in \mathbb{Z}[M]\left[T_{0}, \ldots, T_{r}\right]
$$

by

$$
\begin{aligned}
\Phi_{r}^{(M)}(\boldsymbol{T}) & =\frac{1}{M} \Phi_{r}\left(M T_{0}, \ldots, M T_{r}\right) \\
& =M^{p^{r}-1} T_{0}^{p^{r}}+p M^{p^{r-1}-1} T_{1}^{p^{r-1}}+\cdots+p^{r-1} M^{p-1} T_{r-1}+p^{r} T_{r}
\end{aligned}
$$

Furthermore, we define

$$
\begin{aligned}
& S_{r}^{(M)}(\boldsymbol{X}, \boldsymbol{Y})=S_{r}^{(M)}\left(X_{0}, \ldots, X_{r}, Y_{0}, \ldots, Y_{r}\right) \in \mathbb{Z}[M]\left[X_{0}, \ldots, X_{r}, Y_{0}, \ldots, Y_{r}\right], \\
& P_{r}^{(M)}(\boldsymbol{X}, \boldsymbol{Y})=P_{r}^{(M)}\left(X_{0}, \ldots, X_{r}, Y_{0}, \ldots, Y_{r}\right) \in \mathbb{Z}[M]\left[X_{0}, \ldots, X_{r}, Y_{0}, \ldots, Y_{r}\right] \\
& F_{r}^{(M)}(\boldsymbol{T})=F_{r}^{(M)}\left(T_{0}, \ldots, T_{r}, T_{r+1}\right) \in \mathbb{Z}[M]\left[T_{0}, \ldots, T_{r}, T_{r+1}\right]
\end{aligned}
$$

by

$$
\begin{aligned}
& S_{r}^{(M)}\left(X_{0}, \ldots, X_{r}, Y_{0}, \ldots, Y_{r}\right)=\frac{1}{M} S_{r}\left(M X_{0}, \ldots, M X_{r}, M Y_{0}, \ldots, M Y_{r}\right) \\
& P_{r}^{(M)}\left(X_{0}, \ldots, X_{r}, Y_{0}, \ldots, Y_{r}\right)=\frac{1}{M} P_{r}\left(X_{0}, \ldots, X_{r}, M Y_{0}, \ldots, M Y_{r}\right) \\
& F_{r}^{(M)}(\boldsymbol{T})=F_{r}^{(M)}\left(T_{0}, \ldots, T_{r}, T_{r+1}\right)=\frac{1}{M} F_{r}\left(M T_{0}, \ldots, M T_{r}, M T_{r+1}\right)
\end{aligned}
$$

respectively. For example, we have

$$
\begin{aligned}
& S_{0}^{(M)}(\boldsymbol{X}, \boldsymbol{Y})=X_{0}+Y_{0} \\
& S_{1}^{(M)}(\boldsymbol{X}, \boldsymbol{Y})=X_{1}+Y_{1}+M^{p-1} \frac{X_{0}^{p}+Y_{0}^{p}-\left(X_{0}+Y_{0}\right)^{p}}{p} \\
& P_{0}^{(M)}(\boldsymbol{X}, \boldsymbol{Y})=X_{0} Y_{0} \\
& P_{1}^{(M)}(\boldsymbol{X}, \boldsymbol{Y})=X_{0}^{p} Y_{1}+M^{p-1} X_{1} Y_{0}^{p}+p X_{1} Y_{1} \\
& F_{0}^{(M)}(\boldsymbol{T})=M^{p-1} T_{0}^{p}+p T_{1}
\end{aligned}
$$

We can verify easily that

(1) $\Phi_{r}^{(M)}\left(S_{0}^{(M)}(\boldsymbol{X}, \boldsymbol{Y}), S_{1}^{(M)}(\boldsymbol{X}, \boldsymbol{Y}), \ldots, S_{r}^{(M)}(\boldsymbol{X}, \boldsymbol{Y})\right)=\Phi_{r}^{(M)}(\boldsymbol{X})+\Phi_{r}^{(M)}(\boldsymbol{Y})$;

(2) $\Phi_{r}^{(M)}\left(P_{0}^{(M)}(\boldsymbol{X}, \boldsymbol{Y}), P_{1}^{(M)}(\boldsymbol{X}, \boldsymbol{Y}), \ldots, P_{r}^{(M)}(\boldsymbol{X}, \boldsymbol{Y})\right)=\Phi_{r}(\boldsymbol{X}) \Phi_{r}^{(M)}(\boldsymbol{Y})$;

(3) $\Phi_{r}^{(M)}\left(F_{0}^{(M)}(\boldsymbol{T}), F_{1}^{(M)}(\boldsymbol{T}), \ldots, F_{r}^{(M)}(\boldsymbol{T})\right)=\Phi_{r+1}^{(M)}(\boldsymbol{T})$;

(4) $F_{r}^{(M)}(\boldsymbol{T}) \equiv M^{p-1} T_{r}^{p} \bmod p$. 
Put

$$
\begin{aligned}
S^{(M)}(\boldsymbol{X}, \boldsymbol{Y}) & =\left(S_{0}^{(M)}(\boldsymbol{X}, \boldsymbol{Y}), S_{1}^{(M)}(\boldsymbol{X}, \boldsymbol{Y}), S_{2}^{(M)}(\boldsymbol{X}, \boldsymbol{Y}), \ldots\right) \\
P^{(M)}(\boldsymbol{X}, \boldsymbol{Y}) & =\left(P_{0}^{(M)}(\boldsymbol{X}, \boldsymbol{Y}), P_{1}^{(M)}(\boldsymbol{X}, \boldsymbol{Y}), P_{2}^{(M)}(\boldsymbol{X}, \boldsymbol{Y}), \ldots\right) \\
F^{(M)}(\boldsymbol{T}) & =\left(F_{0}^{(M)}(\boldsymbol{T}), F_{1}^{(M)}(\boldsymbol{T}), P_{2}^{(M)}(\boldsymbol{T}), \ldots\right)
\end{aligned}
$$

Then it follows from (1) (2) (3) that

(5) $S^{(M)}\left(S^{(M)}(\boldsymbol{X}, \boldsymbol{Y}), \boldsymbol{Z}\right)=S^{(M)}\left(\boldsymbol{X}, S^{(M)}(\boldsymbol{Y}, \boldsymbol{Z})\right)$;

(6) $P^{(M)}(P(\boldsymbol{X}, \boldsymbol{Y}), \boldsymbol{Z})=P^{(M)}\left(\boldsymbol{X}, P^{(M)}(\boldsymbol{Y}, \boldsymbol{Z})\right)$;

(7) $F^{(M)}\left(S^{(M)}(\boldsymbol{X}, \boldsymbol{Y})\right)=S^{(M)}\left(F^{(M)}(\boldsymbol{X}), F^{(M)}(\boldsymbol{Y})\right)$.

Furthermore, noting that $\Phi_{r}^{(M)}(\boldsymbol{T}) \equiv p^{r} T_{r} \bmod M^{p-1}$, we can conclude that

(8) $S_{r}^{(M)}(\boldsymbol{X}, \boldsymbol{Y}) \equiv X_{r}+Y_{r} \bmod M^{p-1}$;

(9) $P_{r}^{(M)}(\boldsymbol{X}, \boldsymbol{Y}) \equiv \Phi_{r}(\boldsymbol{X}) Y_{r} \bmod M^{p-1}$;

(10) $F_{r}^{(M)}(\boldsymbol{T}) \equiv p T_{r+1} \bmod M^{p-1}$.

1.5. We define a commutative group scheme $W^{(M)}$ over $\mathbb{Z}[M]$ by

$$
W^{(M)}=\operatorname{Spec} \mathbb{Z}[M]\left[T_{0}, T_{1}, T_{2}, \ldots\right]
$$

with the addition

$$
\begin{aligned}
\boldsymbol{T}= & \left(T_{0}, T_{1}, T_{2}, \ldots\right) \\
& \mapsto\left(S_{0}^{(M)}(\boldsymbol{T} \otimes 1,1 \otimes \boldsymbol{T}), S_{1}^{(M)}(\boldsymbol{T} \otimes 1,1 \otimes \boldsymbol{T}), S_{2}^{(M)}(\boldsymbol{T} \otimes 1,1 \otimes \boldsymbol{T}), \ldots\right) .
\end{aligned}
$$

The unit is given by

$$
\boldsymbol{T}=\left(T_{0}, T_{1}, T_{2}, \ldots\right) \mapsto \mathbf{0}=(0,0,0, \ldots),
$$

and, if $p>2$, the inverse is given by

$$
\boldsymbol{T}=\left(T_{0}, T_{1}, T_{2}, \ldots\right) \mapsto-\boldsymbol{T}=\left(-T_{0},-T_{1},-T_{2}, \ldots\right)
$$

Furthermore, we define a morphism $\cdot: W_{\mathbb{Z}[M]} \times_{\mathbb{Z}[M]} W^{(M)} \rightarrow W^{(M)}$ by

$$
\begin{aligned}
\boldsymbol{T}= & \left(T_{0}, T_{1}, T_{2}, \ldots\right) \\
& \mapsto\left(P_{0}^{(M)}(\boldsymbol{U} \otimes 1,1 \otimes \boldsymbol{T}), P_{1}^{(M)}(\boldsymbol{U} \otimes 1,1 \otimes \boldsymbol{T}), P_{2}^{(M)}(\boldsymbol{U} \otimes 1,1 \otimes \boldsymbol{T}), \ldots\right)
\end{aligned}
$$

Then $W^{(M)}$ is a $W_{\mathbb{Z}[M]}$-module.

Define a morphism of $\mathbb{Z}[M]$-schemes

$$
\Phi^{(M)}: W^{(M)}=\operatorname{Spec} \mathbb{Z}[M]\left[T_{0}, T_{1}, T_{2}, \ldots\right] \rightarrow \mathbb{G}_{a, \mathbb{Z}[M]}^{\mathbb{N}}=\operatorname{Spec} \mathbb{Z}[M]\left[\Phi_{0}, \Phi_{1}, \Phi_{2}, \ldots\right]
$$


by

$$
\left(\Phi_{0}, \Phi_{1}, \Phi_{2}, \ldots\right) \mapsto\left(\Phi_{0}^{(M)}(\boldsymbol{T}), \Phi_{1}^{(M)}(\boldsymbol{T}), \Phi_{2}^{(M)}(\boldsymbol{T}), \ldots\right) .
$$

We have a commutative diagram of $\mathbb{Z}[M]$-schemes

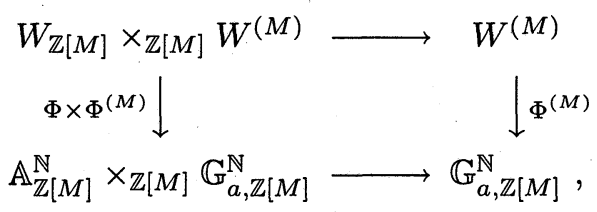

where $\mathbb{A}^{\mathbb{N}} \times_{\mathbb{Z}} \mathbb{G}_{a}^{\mathbb{N}} \rightarrow \mathbb{G}_{a}^{\mathbb{N}}$ is the usual multiplication.

REMARK 1.6. Let $A$ be a $\mathbb{Z}[M]$-algebra. If $p$ is invertible in $A, \Phi: W^{(M)}(A) \rightarrow$ $A^{\mathbb{N}}$ is bijective; if $p$ is a non-zero divisor of $A, \Phi: W^{(M)}(A) \rightarrow A^{\mathbb{N}}$ is injective.

REMARK 1.7. Let $A$ be a $\mathbb{Z}[M]$-algebra. Let $\boldsymbol{a}, \boldsymbol{b} \in W^{(M)}(A)$ and $\boldsymbol{c} \in W(A)$. We will denote sometimes $\boldsymbol{a}+\boldsymbol{b}, \boldsymbol{c} \cdot \boldsymbol{a}$ by $\boldsymbol{a}+{ }^{(M)} \boldsymbol{b}, \boldsymbol{c} \cdot{ }^{(M)} \boldsymbol{a}$, respectively, to avoid confusion.

1.8. Let $A$ be a $\mathbb{Z}[M]$-algebra, and let $\mu$ denote the image of $M$ in $A$. We denote sometimes $W^{(M)} \otimes_{\mathbb{Z}[M]} A$ by $W^{(\mu)}$. We define also

$$
\begin{aligned}
& S_{r}^{(\mu)}(\boldsymbol{X}, \boldsymbol{Y})=S_{r}^{(\mu)}\left(X_{0}, \ldots, X_{r}, Y_{0}, \ldots, Y_{r}\right) \in A\left[X_{0}, \ldots, X_{r}, Y_{0}, \ldots, Y_{r}\right] \\
& P_{r}^{(\mu)}(\boldsymbol{X}, \boldsymbol{Y})=P_{r}^{(\mu)}\left(X_{0}, \ldots, X_{r}, Y_{0}, \ldots, Y_{r}\right) \in A\left[X_{0}, \ldots, X_{r}, Y_{0}, \ldots, Y_{r}\right] \\
& F_{r}^{(\mu)}(\boldsymbol{T})=F_{r}^{(\mu)}\left(T_{0}, \ldots, X_{r}, T_{r+1}\right) \in A\left[T_{0}, \ldots, T_{r}, T_{r+1}\right]
\end{aligned}
$$

by substituting $M$ by $\mu$ in $S_{r}^{(M)}(\boldsymbol{X}, \boldsymbol{Y}), P_{r}^{(M)}(\boldsymbol{X}, \boldsymbol{Y}), F_{r}^{(M)}(\boldsymbol{T})$, respectively.

EXAMPLE 1.9.1. It is clear that

$$
S_{r}^{(1)}(\boldsymbol{X}, \boldsymbol{Y})=S_{r}(\boldsymbol{X}, \boldsymbol{Y}), \quad P_{r}^{(1)}(\boldsymbol{X}, \boldsymbol{Y})=P_{r}(\boldsymbol{X}, \boldsymbol{Y}), \quad F_{r}^{(1)}(\boldsymbol{T})=F_{r}(\boldsymbol{T}),
$$

and therefore $W_{\mathbb{Z}}^{(1)}$ is nothing but the scheme of Witt vectors $W_{\mathbb{Z}}$.

EXAMPLE 1.9.2. It follows from 1.4 (6) (7) (8) that

$$
S_{r}^{(0)}(\boldsymbol{X}, \boldsymbol{Y})=X_{r}+Y_{r}, \quad P_{r}^{(0)}(\boldsymbol{X}, \boldsymbol{Y})=\Phi_{r}(\boldsymbol{X}) Y_{r}, \quad F_{r}^{(0)}(\boldsymbol{T})=p T_{r+1} .
$$

Hence the group scheme $W_{\mathbb{Z}}^{(0)}$ is isomorphic to the direct product $\mathbb{G}_{a, \mathbb{Z}}^{\mathbb{N}}$.

1.10. We define homomorphisms $V: W^{(M)} \rightarrow W^{(M)}$ and $F^{(M)}: W^{(M)} \rightarrow$ $W^{(M)}$ by

$$
\left(T_{0}, T_{1}, T_{2}, \ldots\right) \mapsto\left(0, T_{0}, T_{1}, \ldots\right): \mathbb{Z}[M]\left[T_{0}, T_{1}, T_{2}, \ldots\right] \rightarrow \mathbb{Z}[M]\left[T_{0}, T_{1}, T_{2}, \ldots\right]
$$


and

$$
\begin{aligned}
\left(T_{0}, T_{1}, T_{2}, \ldots\right) \mapsto\left(F_{0}^{(M)}(\boldsymbol{T}), F_{1}^{(M)}(\boldsymbol{T}), F_{2}^{(M)}(\boldsymbol{T}), \ldots\right): & \\
\mathbb{Z}[M]\left[T_{0}, T_{1}, T_{2}, \ldots\right] & \rightarrow \mathbb{Z}[M]\left[T_{0}, T_{1}, T_{2}, \ldots\right],
\end{aligned}
$$

respectively.

Proposition 1.11. Let $A$ be $a \mathbb{Z}[M]$-algebra, $\boldsymbol{a} \in W^{(M)}(A)$ and $\boldsymbol{c} \in W(A)$. Then:

(1) $F^{(M)} \circ V=p$

(2) $F^{(M)}(\boldsymbol{c} \cdot \boldsymbol{a})=F(\boldsymbol{c}) \cdot F^{(M)}(\boldsymbol{a})$;

(3) $V(F(c) \cdot a)=c \cdot V(a)$

(4) $V\left(\boldsymbol{c} \cdot F^{(M)}(\boldsymbol{a})\right)=V(\boldsymbol{c}) \cdot \boldsymbol{a}$.

Proof. To verify the assertion, we may assume

$$
\begin{gathered}
A=\mathbb{Z}[M]\left[U_{0}, U_{1}, U_{2}, \ldots, T_{0}, T_{1}, T_{2}, \ldots\right], \quad \boldsymbol{c}=\boldsymbol{U}=\left(U_{0}, U_{1}, U_{2}, \ldots\right), \\
\boldsymbol{a}=\boldsymbol{T}=\left(T_{0}, T_{1}, T_{2}, \ldots\right) .
\end{gathered}
$$

(1) Note that, for $r \geq 0$,

$$
\Phi_{r}^{(M)}\left(F^{(M)}(V(\boldsymbol{T}))\right)=\Phi_{r+1}^{(M)}(V(\boldsymbol{T}))=p \Phi_{r}^{(M)}(\boldsymbol{T}) .
$$

(2) Note that, for $r \geq 0$,

$$
\Phi_{r}^{(M)}\left(F^{(M)}(\boldsymbol{U} \cdot \boldsymbol{T})\right)=\Phi_{r+1}^{(M)}(\boldsymbol{U} \cdot \boldsymbol{T})=\Phi_{r+1}(\boldsymbol{U}) \Phi_{r+1}^{(M)}(\boldsymbol{T})
$$

and

$\Phi_{r}^{(M)}\left(F(\boldsymbol{U}) \cdot F^{(M)}(\boldsymbol{T})\right)=\Phi_{r}(F(\boldsymbol{U})) \Phi_{r}\left(F^{(M)}(\boldsymbol{T})\right)=\Phi_{r+1}(\boldsymbol{U}) \Phi_{r+1}^{(M)}(\boldsymbol{T})$.

(3) Note that, if $r>0$,

$$
\begin{aligned}
\Phi_{r}^{(M)}(V(F(\boldsymbol{U}) \cdot \boldsymbol{T})) & =p \Phi_{r-1}^{(M)}(F(\boldsymbol{U}) \cdot \boldsymbol{T}) \\
& =p \Phi_{r-1}(F(\boldsymbol{U})) \Phi_{r-1}^{(M)}(\boldsymbol{T})=p \Phi_{r}(\boldsymbol{U}) \Phi_{r-1}^{(M)}(\boldsymbol{T})
\end{aligned}
$$

and

$$
\Phi_{r}^{(M)}(\boldsymbol{U} \cdot V(\boldsymbol{T}))=\Phi_{r}(\boldsymbol{U}) \Phi_{r}^{(M)}(V(\boldsymbol{T}))=\Phi_{r}(\boldsymbol{U}) p \Phi_{r-1}^{(M)}(\boldsymbol{T}) .
$$

(4) Note that, if $r>0$,

$$
\begin{aligned}
\Phi_{r}\left(V\left(\boldsymbol{U} \cdot F^{(M)}(\boldsymbol{T})\right)\right) & =p \Phi_{r-1}^{(M)}\left(\boldsymbol{U} \cdot F^{(M)}(\boldsymbol{T})\right) \\
& =p \Phi_{r-1}(\boldsymbol{U}) \Phi_{r-1}^{(M)}\left(F^{(M)}(\boldsymbol{T})\right)=p \Phi_{r-1}(\boldsymbol{U}) \Phi_{r}^{(M)}(\boldsymbol{T})
\end{aligned}
$$

and

$$
\Phi_{r}^{(M)}(V(\boldsymbol{U}) \cdot \boldsymbol{T})=\Phi_{r}(V(\boldsymbol{U})) \Phi_{r}^{(M)}(\boldsymbol{T})=p \Phi_{r-1}(\boldsymbol{U}) \Phi_{r}^{(M)}(\boldsymbol{T})
$$


1.12. Let $A$ be a $\mathbb{Z}[M]$-algebra. By $1.11(3) \operatorname{Im} V^{k}=\operatorname{Im}\left[V^{k}: W^{(M)}(A) \rightarrow\right.$ $\left.W^{(M)}(A)\right]$ is a sub-W(A)-module of $W^{(M)}(A) . \quad W^{(M)}(A)$ has a structure of a topological group with the $V$-adic topology defined by the family of subgroups $\left\{\operatorname{Im} V^{k}\right\}_{k>0} . W^{(M)}(A)$ is complete and separated for the $V$-adic topology.

1.13. Let $A$ be a $\mathbb{Z}[M]$-algebra and $a \in A$. We denote $(a, 0,0, \ldots) \in$ $W^{(M)}(A)$ by $[a]$.

LeMma 1.13.1. Let $A$ be $\mathbb{Z}[M]$-algebra, $\left(a_{0}, a_{1}, a_{2}, \ldots\right) \in W^{(M)}(A)$, $\left(c_{0}, c_{1}, c_{2}, \ldots\right) \in W(A)$ and $a, c \in A$. Then:

(1) $[c] \cdot\left(a_{0}, a_{1}, a_{2}, \ldots\right)=\left(c a_{0}, c^{p} a_{1}, c^{p^{2}} a_{2}, \ldots\right)$;

(2) $\left(c_{0}, c_{1}, c_{2}, \ldots\right) \cdot[a]=\left(c_{0} a, M^{p-1} c_{1} a^{p}, M^{p^{2}-1} c_{2} a^{p^{2}}, \ldots\right)$;

(3) $F^{(M)}([a])=\left[M^{p-1} a^{p}\right]$.

Proof. (1) We may assume that $A=\mathbb{Z}[M]\left[U, T_{0}, T_{1}, T_{2}, \ldots\right], c=U$ and $\left(a_{0}, a_{1}, a_{2}, \ldots\right)=\left(T_{0}, T_{1}, T_{2}, \ldots\right)$. Now note that

$$
\begin{aligned}
\Phi_{r}^{(M)} & \left([U] \cdot\left(T_{0}, T_{1}, T_{2}, \ldots\right)\right)=\Phi_{r}(U, 0, \ldots, 0) \Phi_{r}^{(M)}\left(T_{0}, T_{1}, T_{2}, \ldots, T_{r}\right) \\
& =U^{p^{r}}\left\{M^{p^{r}-1} T_{0}^{p^{r}}+p M^{p^{r-1}-1}\left(T_{1}\right)^{p^{r-1}}+p^{2} M^{p^{r-2}-1}\left(T_{2}\right)^{p^{r-2}}+\cdots+p^{r} T_{r}\right\}
\end{aligned}
$$

and

$$
\begin{aligned}
\Phi_{r}^{(M)}\left(U T_{0}, U^{p} T_{1}, U^{p^{2}} T_{2}, \ldots, U^{p^{r}} T_{r}\right) & \\
=M^{p^{r}-1}\left(U T_{0}\right)^{p^{r}}+p M^{p^{r-1}-1}\left(U^{p} T_{1}\right)^{p^{r-1}}+p M^{p^{r-2}-1}\left(U^{p^{2}} T_{2}\right)^{p^{r-2}} & +\cdots+p^{r}\left(U^{p^{r}} T_{r}\right) \\
& +\cdots
\end{aligned}
$$

Hence the result.

(2) We may assume that $A=\mathbb{Z}[M]\left[U_{0}, U_{1}, U_{2}, \ldots, T\right],\left(c_{0}, c_{1}, c_{2}, \ldots\right)=$ $\left(U_{0}, U_{1}, U_{2}, \ldots\right)$ and $a=T$. Now note that

$$
\begin{aligned}
\Phi_{r}^{(M)}\left(\left(U_{0}, U_{1}, U_{2}, \ldots\right) \cdot[T]\right) & =\Phi_{r}\left(U_{0}, U_{1}, U_{2}, \ldots, U_{r}\right) \Phi_{r}^{(M)}(T, 0, \ldots, 0) \\
& =\left(U_{0}^{p^{r}}+p U_{1}^{p^{r-1}}+p^{2} U_{2}^{p^{r-2}}+\cdots+p^{r} U_{r}\right)\left(M^{p^{r}-1} T^{p^{r}}\right)
\end{aligned}
$$

and

$$
\begin{aligned}
& \Phi_{r}^{(M)}\left(U_{0} T, M^{p-1} U_{1} T^{p}, M^{p^{2}-1} U_{2} T^{p^{2}}, \ldots, M^{p^{r}-1} U_{r} T^{p^{r}}\right) \\
& =M^{p^{r}-1}\left(U_{0} T\right)^{p^{r}}+p M^{p^{r-1}-1}\left(M^{p-1} U_{1} T^{p}\right)^{p^{r-1}} \\
& \quad+p^{2} M^{p^{r-2}-1}\left(M^{p^{2}-1} U_{2} T^{p^{2}}\right)^{p^{r-2}}+\cdots+p^{r}\left(M^{p^{r}-1} U_{r} T^{p^{r}}\right) .
\end{aligned}
$$

Hence the result. 
(3) Note that, for $r \geq 0$,

$$
\begin{aligned}
\Phi_{r}^{(M)}\left(F^{(M)}([T])\right) & =\Phi_{r+1}^{(M)}([T])=M^{p^{r+1}-1} T^{p^{r+1}} \\
& =M^{p^{r}-1}\left(M^{p-1} T^{p}\right)^{p^{r}}=\Phi_{r}^{(M)}\left(\left[M^{p-1} T^{p}\right]\right) .
\end{aligned}
$$

REMARK 1.13.2. We will denote sometimes $[a] \in W^{(M)}(A)$ by $[a]_{M}$ to avoid confusion.

LEMMA 1.14. Let $A$ be a $\mathbb{Z}[M]$-algebra and $\boldsymbol{a}=\left(a_{0}, a_{1}, a_{2}, \ldots\right) \in W^{(M)}(A)$. Then we have

$$
\boldsymbol{a}=\sum_{k=0}^{\infty} V^{k}\left[a_{k}\right]
$$

Proof. We may assume that $A=\mathbb{Z}[M]\left[T_{0}, T_{1}, T_{2}, \ldots\right]$ and $\left(a_{0}, a_{1}, a_{2}, \ldots\right)=$ $\left(T_{0}, T_{1}, T_{2}, \ldots\right)$. Then we can verify the equality, noting that

$$
\begin{aligned}
\Phi_{r}^{(M)}\left(\sum_{k=0}^{\infty} V^{k}\left[T_{k}\right]\right) & =\sum_{k=0}^{r} \Phi_{r}^{(M)}\left(V^{k}\left[T_{k}\right]\right)=\sum_{k=0}^{r} p^{k} \Phi_{r-k}^{(M)}\left(\left[T_{k}\right]\right) \\
& =\Phi_{r}^{(M)}\left(T_{0}, T_{1}, \ldots, T_{r}\right)
\end{aligned}
$$

1.15. We define a morphism $\alpha^{(M)}: W^{(M)} \rightarrow W_{\mathbb{Z}[M]}$ by

$$
\begin{gathered}
\left(T_{0}, T_{1}, T_{2}, \ldots\right) \mapsto\left(M T_{0}, M T_{1}, M T_{2}, \ldots\right): \\
\mathbb{Z}[M]\left[T_{0}, T_{1}, T_{2}, \ldots\right] \rightarrow \mathbb{Z}[M]\left[T_{0}, T_{1}, T_{2}, \ldots\right] .
\end{gathered}
$$

By $1.4(1)$

$$
\begin{aligned}
M \Phi_{r}^{(M)}\left(S_{0}^{(M)}(\boldsymbol{X}, \boldsymbol{Y}), S_{1}^{(M)}(\boldsymbol{X}, \boldsymbol{Y}), \ldots, S_{r}^{(M)}(\boldsymbol{X}, \boldsymbol{Y})\right) \\
=\Phi_{r}\left(S_{0}\left(M X_{0}, M Y_{0}\right), S_{1}\left(M X_{0}, M X_{1}, M Y_{0}, M Y_{1}\right), \ldots\right. \\
\left.S_{r}\left(M X_{0}, \ldots, M X_{r}, M Y_{0}, \ldots, M Y_{r}\right)\right)
\end{aligned}
$$

for $r \geq 0$. Therefore $\alpha^{(M)}: W^{(M)} \rightarrow W_{\mathbb{Z}[M]}$ is a homomorphism of group schemes. Furthermore we have gotten a commutative diagram of group schemes over $\mathbb{Z}[M]$ :

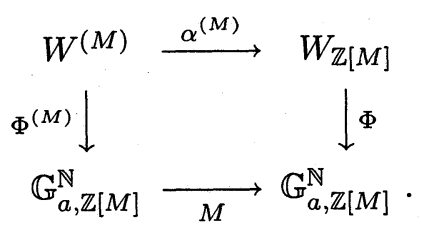


Here

$M: \mathbb{G}_{a, \mathbb{Z}[M]}^{\mathbb{N}}=\operatorname{Spec} \mathbb{Z}[M]\left[\Phi_{0}, \Phi_{1}, \Phi_{2}, \ldots\right] \rightarrow \mathbb{G}_{a, \mathbb{Z}[M]}^{\mathbb{N}}=\operatorname{Spec} \mathbb{Z}[M]\left[\Phi_{0}, \Phi_{1}, \Phi_{2}, \ldots\right]$

is defined by

$$
\left(\Phi_{0}, \Phi_{1}, \Phi_{2}, \ldots\right) \mapsto\left(M \Phi_{0}, M \Phi_{1}, M \Phi_{2}, \ldots\right)
$$

Proposition 1.16. Let $A$ be a $\mathbb{Z}[M]$-algebra and $\boldsymbol{a} \in W^{(M)}(A), \boldsymbol{c} \in W(A)$. Then:

(1) $\alpha^{(M)}(\boldsymbol{c} \cdot \boldsymbol{a})=\boldsymbol{c} \cdot \alpha^{(M)}(\boldsymbol{a})$;

(2) $\alpha^{(M)}\left(F^{(M)}(a)\right)=F\left(\alpha^{(M)}(a)\right)$;

(3) $\alpha^{(M)}(V(\boldsymbol{a}))=V\left(\alpha^{(M)}(\boldsymbol{a})\right)$.

Proof. (3) is trivial. To prove (1) and (2), we may assume

$$
\begin{gathered}
A=\mathbb{Z}[M]\left[U_{0}, U_{1}, U_{2}, \ldots, T_{0}, T_{1}, T_{2}, \ldots\right], \quad \boldsymbol{c}=\boldsymbol{U}=\left(U_{0}, U_{1}, U_{2}, \ldots\right), \\
\boldsymbol{a}=\boldsymbol{T}=\left(T_{0}, T_{1}, T_{2}, \ldots\right) .
\end{gathered}
$$

(1) Note that, for $r \geq 0$,

$$
\Phi_{r}\left(\alpha^{(M)}(\boldsymbol{U} \cdot \boldsymbol{T})\right)=M \Phi_{r}^{(M)}(\boldsymbol{U} \cdot \boldsymbol{T})=M \Phi_{r}(\boldsymbol{U}) \Phi_{r}^{(M)}(\boldsymbol{T})
$$

and

$$
\Phi_{r}\left(\boldsymbol{U} \cdot \alpha^{(M)}(\boldsymbol{T})\right)=\Phi_{r}(\boldsymbol{U}) \Phi_{r}\left(\alpha^{(M)}(\boldsymbol{T})\right)=\Phi_{r}(\boldsymbol{U}) M \Phi_{r}^{(M)}(\boldsymbol{T}) .
$$

(2) Note that, for $r \geq 0$,

$$
\begin{aligned}
\Phi_{r}\left(\alpha^{(M)}\left(F^{(M)}(\boldsymbol{T})\right)\right) & =M \Phi_{r}^{(M)}\left(F^{(M)}(\boldsymbol{T})\right)=M \Phi_{r+1}^{(M)}(\boldsymbol{T}) \\
& =\Phi_{r+1}\left(\alpha^{(M)}(\boldsymbol{T})\right)=\Phi_{r}\left(F\left(\alpha^{(M)}(\boldsymbol{T})\right)\right) .
\end{aligned}
$$
$W_{n}^{(M)}$ by

REMARK 1.17. As in the classical theory of Witt vectors, we can define

$$
W_{n}^{(M)}=\operatorname{Coker}\left[V^{n}: W^{(M)} \rightarrow W^{(M)}\right] .
$$

We have gotten systems of morphisms $V$ and $F^{(M)}$ and several formulae. For example, we have commutative diagrams

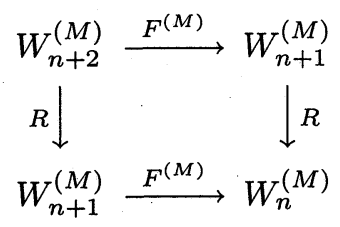


and

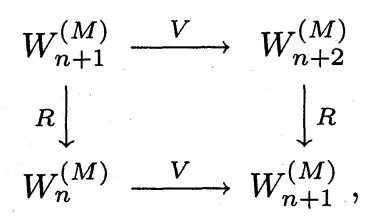

where $R$ denotes the canonical surjection.

We conclude the section, giving another description of $W^{(M)}$.

1.18. Let $A$ be a $\mathbb{Z}[M]$-algebra and $B=A[t] /\left(t^{2}-M t\right)$. Let $\varepsilon$ denote the image of $t$ in $B$. Then we have $\varepsilon^{2}=M \varepsilon$. Defining a ring homomorphism $B \rightarrow A$ by $\varepsilon \mapsto 0$, we have also a ring homomorphism $W(B) \rightarrow W(A)$ and

$$
\operatorname{Ker}[W(B) \rightarrow W(A)]=\left\{\left(\varepsilon a_{0}, \varepsilon a_{1}, \varepsilon a_{2}, \ldots\right) ; a_{0}, a_{1}, a_{2}, \ldots \in A\right\} .
$$

LEMma 1.19. Let $B=\mathbb{Z}[M, t] /\left(t^{2}-M t\right)$, and let $\varepsilon$ denote the image of $t$ in B. Then:

(1) $\Phi_{r}\left(\varepsilon T_{0}, \varepsilon T_{1}, \ldots, \varepsilon T_{r}\right)=\varepsilon \Phi_{r}^{(M)}\left(T_{0}, T_{1}, \ldots, T_{r}\right)$;

(2) $\varepsilon S_{r}^{(M)}(\boldsymbol{X}, \boldsymbol{Y})=S_{r}\left(\varepsilon X_{0}, \varepsilon X_{1}, \ldots, \varepsilon X_{r}, \varepsilon Y_{0}, \varepsilon Y_{1}, \ldots, \varepsilon Y_{r}\right)$;

(3) $\varepsilon P_{r}^{(M)}(\boldsymbol{X}, \boldsymbol{Y})=P_{r}\left(X_{0}, X_{1}, \ldots, X_{r}, \varepsilon Y_{0}, \varepsilon Y_{1}, \ldots, \varepsilon Y_{r}\right)$;

(4) $\varepsilon F_{r}^{(M)}(\boldsymbol{T})=F_{r}\left(\varepsilon T_{0}, \ldots, \varepsilon T_{r}, \varepsilon T_{r+1}\right)$.

Proof. The assertion is a direct consequence of the following lemma.

LemMA 1.20. Let $A$ be $a$ ring and $B=A[M, t] /\left(t^{2}-M t\right)$, and let $\varepsilon$ denote the image of $t$ in $B$. Let $f\left(T_{1}, T_{2}, \ldots, T_{n}\right) \in A\left[T_{1}, T_{2}, \ldots, T_{n}\right]$ with $f(0,0, \ldots, 0)=$ 0 , and put

$$
f^{(M)}\left(T_{1}, T_{2}, \ldots, T_{n}\right)=\frac{1}{M} f\left(M T_{1}, M T_{2}, \ldots, M T_{n}\right) .
$$

Then $f^{(M)}\left(T_{1}, T_{2}, \ldots, T_{n}\right) \in A[M]\left[T_{1}, T_{2}, \ldots, T_{n}\right]$ and

$$
\varepsilon f^{(M)}\left(T_{1}, T_{2}, \ldots, T_{n}\right)=f\left(\varepsilon T_{1}, \varepsilon T_{2}, \ldots, \varepsilon T_{n}\right) .
$$

Proof. It is sufficient to verify the assertion in the case where

$$
f\left(T_{1}, T_{2}, \ldots, T_{n}\right)=T_{1}^{i_{1}} T_{2}^{i_{2}} \cdots T_{n}^{i_{n}}
$$

with $i_{1}+i_{2}+\cdots+i_{n}>0$. In this case, we have

$$
f^{(M)}\left(T_{1}, T_{2}, \ldots, T_{n}\right)=M^{i_{1}+i_{2}+\cdots+i_{n}-1} T_{1}^{i_{1}} T_{2}^{i_{2}} \cdots T_{n}^{i_{n}},
$$


and therefore,

$$
\begin{aligned}
\varepsilon f^{(M)}\left(T_{1}, T_{2}, \ldots, T_{n}\right) & =\varepsilon M^{i_{1}+i_{2}+\cdots+i_{n}-1} T_{1}^{i_{1}} T_{2}^{i_{2}} \cdots T_{n}^{i_{n}} \\
& =\varepsilon^{i_{1}+i_{2}+\cdots+i_{n}} T_{1}^{i_{1}} T_{2}^{i_{2}} \cdots T_{n}^{i_{n}}=\left(\varepsilon T_{1}\right)^{i_{1}}\left(\varepsilon T_{2}\right)^{i_{2}} \cdots\left(\varepsilon T_{n}\right)^{i_{n}}
\end{aligned}
$$

since $M^{k-1} \varepsilon=\varepsilon^{k}$ for $k \geq 1$.

Combining 1.18 and 1.19 , we obtain the following:

Theorem 1.21. Let $A=\mathbb{Z}[M], B=\mathbb{Z}[M, t] /\left(t^{2}-M t\right)$. Then $W^{(M)}$ is isomorphic to $\operatorname{Ker}\left[\prod_{B / A} W_{B} \rightarrow W_{A}\right]$, where $\prod_{B / A}$ denotes the Weil restriction functor. More precisely,

(1) $\left(a_{0}, a_{1}, a_{2}, \ldots\right) \mapsto\left(\varepsilon a_{0}, \varepsilon a_{1}, \varepsilon a_{2}, \ldots\right)$ gives rise to a $W(A)$-isomorphism

$$
W^{(M)}(A) \stackrel{\sim}{\rightarrow} \operatorname{Ker}[W(B) \rightarrow W(A)]
$$

(2) $F: W(B) \rightarrow W(B)$ induces $F^{(M)}$ on $W^{(M)}(A)$;

(3) $V: W(B) \rightarrow W(B)$ induces $V$ on $W^{(M)}(A)$.

\section{Around the Artin-Hasse exponential series}

We first recall the necessary facts on the Artin-Hasse exponential series. For details, see $[2, \mathrm{Ch} . \mathrm{V}]$ or [4].

2.1. The Artin-Hasse exponential series $E_{p}(U) \in \mathbb{Z}_{(p)}[[U]]$ is defined by

$$
E_{p}(T)=\exp \left(\sum_{r \geq 0} \frac{T^{p^{r}}}{p^{r}}\right)
$$

For $\boldsymbol{U}=\left(U_{r}\right)_{r \geq 0}$, we put

$$
E_{p}(\boldsymbol{U} ; T)=\prod_{r \geq 0} E_{p}\left(U_{r} T^{p^{r}}\right)=\exp \left(\sum_{r \geq 0} \frac{\Phi_{r}(\boldsymbol{U}) T^{p^{r}}}{p^{r}}\right) .
$$

It is readily seen that

$$
E_{p}(S(\boldsymbol{U}, \boldsymbol{V}) ; T)=E_{p}(\boldsymbol{U} ; T) E_{p}(\boldsymbol{V} ; T)
$$

2.2. In [13, Sect. 2] we defined a formal power series $E_{p}(U, \Lambda ; T) \in$ $\mathbb{Z}_{(p)}[U, \Lambda][[T]]$ by

$$
E_{p}(U, \Lambda ; T)=(1+\Lambda T)^{\frac{U}{\Lambda}} \prod_{r=1}^{\infty}\left(1+\Lambda^{p^{r}} T^{p^{r}}\right)^{\frac{1}{p^{r}}\left\{\left(\frac{U}{\Lambda}\right)^{p^{r}}-\left(\frac{U}{\Lambda}\right)^{p^{r-1}}\right\}}
$$


Then we have the equalities:

$$
\log E_{p}(U, \Lambda ; T)=\sum_{r=0}^{\infty} \frac{1}{p^{r}}\left(\frac{U}{\Lambda}\right)^{p^{r}}\left\{\log \left(1+\Lambda^{p^{r}} T^{p^{r}}\right)-\frac{1}{p} \log \left(1+\Lambda^{p^{r+1}} T^{p^{r+1}}\right)\right\}
$$

and

$$
E_{p}(U, \Lambda ; T)= \begin{cases}\prod_{(k, p)=1} E_{p}\left(U \Lambda^{k-1} T^{k}\right)^{(-1)^{k-1} / k} & \text { if } p>2 \\ \prod_{(k, 2)=1} E_{p}\left(U \Lambda^{k-1} T^{k}\right)^{1 / k}\left[\prod_{(k, 2)=1} E_{p}\left(U \Lambda^{2 k-1} T^{2 k}\right)^{1 / k}\right]^{-1} & \text { if } p=2\end{cases}
$$

([13, Th. 2.4]).

For $\boldsymbol{U}=\left(U_{r}\right)_{r \geq 0}$, we put

$$
\begin{aligned}
E_{p}(\boldsymbol{U}, \Lambda ; T) & =\prod_{r \geq 0} E_{p}\left(U_{r}, \Lambda^{p^{r}} ; T^{p^{r}}\right) \\
& =(1+\Lambda T)^{\frac{\Phi_{0}(U)}{\Lambda}} \prod_{k=1}^{\infty}\left(1+\Lambda^{p^{k}} T^{p^{k}}\right)^{\frac{1}{p^{k} \Lambda^{p^{k}}}\left\{\Phi_{k}(U)-\Lambda^{p^{k-1}(p-1)} \Phi_{k-1}(\boldsymbol{U})\right\}}
\end{aligned}
$$

Then we obtain

$$
\log E_{p}(\boldsymbol{U}, \Lambda ; T)=\sum_{r=0}^{\infty} \frac{\Phi_{r}(\boldsymbol{U})}{p^{r} \Lambda^{p^{r}}}\left\{\log \left(1+\Lambda^{p^{r}} T^{p^{r}}\right)-\frac{1}{p} \log \left(1+\Lambda^{p^{r+1}} T^{p^{r+1}}\right)\right\}
$$

and

$$
\begin{aligned}
& E_{p}(\boldsymbol{U}, \Lambda ; T) \\
& = \begin{cases}\prod_{(k, p)=1} E_{p}\left(\boldsymbol{U} ; \Lambda^{k-1} T^{k}\right)^{(-1)^{k-1} / k} & \text { if } p>2 \\
\prod_{(k, 2)=1} E_{p}\left(\boldsymbol{U} ; \Lambda^{k-1} T^{k}\right)^{1 / k}\left[\prod_{(k, 2)=1} E_{p}\left(\boldsymbol{U} ; \Lambda^{2 k-1} T^{2 k}\right)^{1 / k}\right]^{-1} & \text { if } p=2 .\end{cases}
\end{aligned}
$$

It is verified without difficulty that

$$
E_{p}(S(\boldsymbol{U}, \boldsymbol{V}), \Lambda ; T)=E_{p}(\boldsymbol{U}, \Lambda ; T) E_{p}(\boldsymbol{V}, \Lambda ; T)
$$

([13, Cor. 2.9]).

EXAMPLE 2.2.1. $E_{p}(\boldsymbol{U}, 0 ; T)=E_{p}(\boldsymbol{U} ; T)$.

EXAMPle 2.2.2. $E_{p}([\Lambda], \Lambda ; T)=1+\Lambda T$. 
2.3. For $\boldsymbol{U}=\left(U_{0}, U_{1}, U_{2}, \ldots\right)$, we define $E_{p}^{(M)}(\boldsymbol{U}, \Lambda ; T) \in \mathbb{Z}_{(p)}\left[\Lambda, M, U_{0}, U_{1}, U_{2}, \ldots\right][[T]]$ by

$$
E_{p}^{(M)}(\boldsymbol{U}, \Lambda ; T)=\frac{1}{M}\left[E_{p}\left(\alpha^{(M)} \boldsymbol{U}, \Lambda ; T\right)-1\right] .
$$

Example 2.3.1. $E_{p}^{(1)}(\boldsymbol{U}, \Lambda ; T)=E_{p}(\boldsymbol{U}, \Lambda ; T)-1$.

EXAMPLE 2.3.2.

$$
E_{p}^{(0)}(\boldsymbol{U}, \Lambda ; T)=\sum_{r=0}^{\infty} \frac{U_{r}}{\Lambda^{p^{r}}}\left\{\log \left(1+\Lambda^{p^{r}} T^{p^{r}}\right)-\frac{1}{p} \log \left(1+\Lambda^{p^{r+1}} T^{p^{r+1}}\right)\right\} .
$$

Indeed, by the definition we have

$$
1+M E_{p}^{(M)}(\boldsymbol{U}, \Lambda ; T)=E_{p}\left(\alpha^{(M)} \boldsymbol{U}, \Lambda ; T\right)
$$

and

$$
\begin{aligned}
& \log E_{p}\left(\alpha^{(M)} \boldsymbol{U}, \Lambda ; T\right) \\
& =\sum_{r=0}^{\infty} \frac{\Phi_{r}\left(M U_{0}, M U_{1}, \ldots, M U_{r}\right)}{p^{r} \Lambda^{p^{r}}}\left\{\log \left(1+\Lambda^{p^{r}} T^{p^{r}}\right)-\frac{1}{p} \log \left(1+\Lambda^{p^{r+1}} T^{p^{r+1}}\right)\right\} .
\end{aligned}
$$

Now note that, for $r \geq 0$,

$$
\Phi_{r}\left(M U_{0}, M U_{1}, \ldots, M U_{r}\right) \equiv p^{r} M U_{r} \bmod M^{p}
$$

Hence we have

$$
\log E_{p}\left(\alpha^{(M)} \boldsymbol{U}, \Lambda ; T\right) \equiv \sum_{r=0}^{\infty} \frac{M U_{r}}{\Lambda^{p^{r}}}\left\{\log \left(1+\Lambda^{p^{r}} T^{p^{r}}\right)-\frac{1}{p} \log \left(1+\Lambda^{p^{r+1}} T^{p^{r+1}}\right)\right\}
$$

$\bmod M^{p}$,

and therefore

$$
\begin{array}{r}
E_{p}\left(\alpha^{(M)} \boldsymbol{U}, \Lambda ; T\right) \equiv 1+\sum_{r=0}^{\infty} \frac{M U_{r}}{\Lambda^{p^{r}}}\left\{\log \left(1+\Lambda^{p^{r}} T^{p^{r}}\right)-\frac{1}{p} \log \left(1+\Lambda^{p^{r+1}} T^{p^{r+1}}\right)\right\} \\
\bmod M^{2} .
\end{array}
$$

Thus we obtain

$$
E_{p}^{(M)}(\boldsymbol{U}, \Lambda ; T) \equiv \sum_{r=0}^{\infty} \frac{U_{r}}{\Lambda^{p^{r}}}\left\{\log \left(1+\Lambda^{p^{r}} T^{p^{r}}\right)-\frac{1}{p} \log \left(1+\Lambda^{p^{r+1}} T^{p^{r+1}}\right)\right\} \bmod M .
$$


EXAMPLE 2.3.3. $E_{p}^{(M)}(\boldsymbol{U}, 0 ; T)=\frac{1}{M}\left[\prod_{r=0}^{\infty} E_{p}\left(M U_{r} ; T^{p^{r}}\right)-1\right]$.

Proposition 2.4. Let $\boldsymbol{U}=\left(U_{0}, U_{1}, U_{2}, \ldots\right), \boldsymbol{V}=\left(V_{0}, V_{1}, V_{2}, \ldots\right)$. Then we have

$$
\begin{aligned}
& E_{p}^{(M)}\left(\boldsymbol{U}+{ }^{(M)} \boldsymbol{V}, \Lambda ; T\right) \\
& \quad=E_{p}^{(M)}(\boldsymbol{U}, \Lambda ; T)+E_{p}^{(M)}(\boldsymbol{V}, \Lambda ; T)+M E_{p}^{(M)}(\boldsymbol{U}, \Lambda ; T) E_{p}^{(M)}(\boldsymbol{V}, \Lambda ; T)
\end{aligned}
$$

Proof. It is sufficient to prove that

$$
\left[1+M E_{p}^{(M)}(\boldsymbol{U}, \Lambda ; T)\right]\left[1+M E_{p}^{(M)}(\boldsymbol{V}, \Lambda ; T)\right]=1+M E_{p}^{(M)}\left(\boldsymbol{U}+{ }^{(M)} \boldsymbol{V}, \Lambda ; T\right),
$$

that is to say,

$$
E_{p}\left(\alpha^{(M)} \boldsymbol{U}, \Lambda ; T\right) E_{p}\left(\alpha^{(M)} \boldsymbol{V}, \Lambda ; T\right)=E_{p}\left(\alpha^{(M)}\left(\boldsymbol{U}+{ }^{(M)} \boldsymbol{V}\right), \Lambda ; T\right) .
$$

This is a consequence of the functional equation for $E_{p}(\boldsymbol{U}, \Lambda ; T)$ since $\alpha^{(M)}(\boldsymbol{U})+$ $\alpha^{(M)}(\boldsymbol{V})=\alpha^{(M)}\left(\boldsymbol{U}+{ }^{(M)} \boldsymbol{V}\right)$.

We conclude the section by introducing formal power series to describe 2cocycles.

2.5. In $[13,2.15]$ we defined a formal power series

$$
F_{p}(\boldsymbol{U}, \Lambda ; X, Y) \in \mathbb{Z}_{(p)}\left[U_{0}, U_{1}, U_{2}, \ldots, \Lambda\right][[X, Y]]
$$

by

$$
F_{p}(\boldsymbol{U}, \Lambda ; X, Y)=\prod_{r=0}^{\infty}\left[\frac{\left(1+\Lambda^{p^{r+1}} X^{p^{r+1}}\right)\left(1+\Lambda^{p^{r+1}} Y^{p^{r+1}}\right)}{1+\Lambda^{p^{r+1}}(X+Y+\Lambda X Y)^{p^{r+1}}}\right]^{\Phi_{r}(\boldsymbol{U}) / p^{r+1} \Lambda^{p^{r+1}}}
$$

It is readily seen that

(1) $F_{p}(\boldsymbol{U}, \Lambda ; X, Y) F_{p}(\boldsymbol{U}, \Lambda ; X+Y+\Lambda X Y, Z)$

$$
=F_{p}(\boldsymbol{U}, \Lambda ; X, Y+Z+\Lambda Y Z) F_{p}(\boldsymbol{U}, \Lambda ; Y, Z),
$$

(2) $F_{p}(\boldsymbol{U}, \Lambda ; X, Y)=F_{p}(\boldsymbol{U}, \Lambda ; Y, X)$.

Moreover, we have

(3) $F_{p}(S(\boldsymbol{T}, \boldsymbol{U}), \Lambda ; X, Y)=F_{p}(\boldsymbol{T}, \Lambda ; X, Y) F_{p}(\boldsymbol{U}, \Lambda ; X, Y)$.

REMARK 2.6. In [12, Sect. 2] we defined a formal power series

$$
F_{p}(\boldsymbol{U} ; X, Y)=F_{p, 1}(\boldsymbol{U} ; X, Y) \in \mathbb{Z}_{(p)}\left[U_{0}, U_{1}, U_{2}, \ldots\right][X, Y]
$$


by

$$
F_{p}(\boldsymbol{U} ; X, Y)=\exp \left(\sum_{r=0}^{\infty} \Phi_{r}(\boldsymbol{U}) \frac{X^{p^{r+1}}+Y^{p^{r+1}}-(X+Y)^{p^{r+1}}}{p^{r+1}}\right) .
$$

We can verify without difficulty that

$$
F_{p}(\boldsymbol{U}, 0 ; X, Y)=F_{p, 1}(\boldsymbol{U} ; X, Y)
$$

2.7. Now define a formal power series

$$
F_{p}^{(M)}(\boldsymbol{U}, \Lambda ; X, Y) \in \mathbb{Z}_{(p)}\left[\Lambda, M, U_{0}, U_{1}, U_{2}, \ldots\right][[X, Y]]
$$

by

$$
F_{p}^{(M)}(\boldsymbol{U}, \Lambda ; X, Y)=\frac{1}{M}\left[F_{p}\left(\alpha^{(M)} \boldsymbol{U}, \Lambda ; X, Y\right)-1\right]
$$

EXAMPLE 2.7.1. $F_{p}^{(1)}(\boldsymbol{U}, \Lambda ; X, Y)=F_{p}(\boldsymbol{U}, \Lambda ; X, Y)-1 . \quad$ In particular, $F_{p}^{(1)}(\boldsymbol{U}, 0 ; X, Y)=F_{p}(\boldsymbol{U} ; X, Y)-1$.

EXAMPLE 2.7.2.

$$
F_{p}^{(0)}(\boldsymbol{U}, \Lambda ; X, Y)=\sum_{r=0}^{\infty} \frac{U_{r}}{p \Lambda^{p^{r+1}}} \log \frac{\left(1+\Lambda^{p^{r+1}} X^{p^{r+1}}\right)\left(1+\Lambda^{p^{r+1}} Y^{p^{r+1}}\right)}{1+\Lambda^{p^{r+1}}(X+Y+\Lambda X Y)^{p^{r+1}}}
$$

Indeed, by the definition we have

$$
1+M F_{p}^{(M)}(\boldsymbol{U}, \Lambda ; X, Y)=F_{p}\left(\alpha^{(M)}(\boldsymbol{U}), \Lambda ; X, Y\right)
$$

and

$$
\begin{aligned}
& \log F_{p}\left(\alpha^{(M)} \boldsymbol{U}, \Lambda ; X, Y\right) \\
& \quad=\sum_{r=0}^{\infty} \frac{\Phi_{r}\left(M U_{0}, M U_{1}, \ldots, M U_{r}\right)}{p^{r+1} \Lambda^{p^{r+1}}} \log \left[\frac{\left(1+\Lambda^{p^{r+1}} X^{p^{r+1}}\right)\left(1+\Lambda^{p^{r+1}} Y^{p^{r+1}}\right)}{1+\Lambda^{p^{r+1}}(X+Y+\Lambda X Y)^{p^{r+1}}}\right] .
\end{aligned}
$$

Hence, as in Example 2.3.2, we obtain

$$
\begin{aligned}
\log F_{p}\left(\alpha^{(M)} \boldsymbol{U}, \Lambda ; X, Y\right) \\
\quad \equiv \sum_{r=0}^{\infty} \frac{M U_{r}}{p \Lambda^{p^{r+1}}} \log \left[\frac{\left(1+\Lambda^{p^{r+1}} X^{p^{r+1}}\right)\left(1+\Lambda^{p^{r+1}} Y^{p^{r+1}}\right)}{1+\Lambda^{p^{r+1}}(X+Y+\Lambda X Y)^{p^{r+1}}}\right] \bmod M^{p}
\end{aligned}
$$

which implies the assertion.

In particular, putting $\boldsymbol{U}=[1]_{0}$ and $\Lambda=0$, we obtain

$$
F_{p}^{(0)}\left([1]_{0}, 0 ; X, Y\right)=\frac{X^{p}+Y^{p}-(X+Y)^{p}}{p}
$$


which is the 2-cocycle of $Z^{2}\left(\widehat{\mathbb{G}}_{a}, \widehat{\mathbb{G}}_{a}\right)$ defining the extension $\widehat{W}_{2}$ or the 2-cocycle of $Z^{2}\left(\mathbb{G}_{a}, \mathbb{G}_{a}\right)$ defining the extension $W_{2}$.

Proposition 2.8. Let $\boldsymbol{U}=\left(U_{0}, U_{1}, U_{2}, \ldots\right)$. Then

$$
\begin{aligned}
& F_{p}^{(M)}(\boldsymbol{U}, \Lambda ; X, Y)+F_{p}^{(M)}(\boldsymbol{U}, \Lambda ; X+Y+\Lambda X Y, Z) \\
& +M F_{p}^{(M)}(\boldsymbol{U}, \Lambda ; X, Y) F_{p}^{(M)}(\boldsymbol{U}, \Lambda ; X+Y+\Lambda X Y, Z) \\
& =F_{p}^{(M)}(\boldsymbol{U}, \Lambda ; X, Y+Z+\Lambda Y Z)+F_{p}^{(M)}(\boldsymbol{U}, \Lambda ; Y, Z) \\
& \quad+M F_{p}^{(M)}(\boldsymbol{U}, \Lambda ; X, Y+Z+\Lambda Y Z) F_{p}^{(M)}(\boldsymbol{U}, \Lambda ; Y, Z)
\end{aligned}
$$

and

$$
F_{p}^{(M)}(\boldsymbol{U}, \Lambda ; X, Y)=F_{p}^{(M)}(\boldsymbol{U}, \Lambda ; Y, X)
$$

Proof. To verify the first assertion, it is sufficient to prove that

$$
\begin{aligned}
& {\left[1+M F_{p}^{(M)}(\boldsymbol{U}, \Lambda ; X, Y)\right]\left[1+M F_{p}^{(M)}(\boldsymbol{U}, \Lambda ; X+Y+\Lambda X Y, Z)\right]} \\
& =\left[1+M F_{p}^{(M)}(\boldsymbol{U}, \Lambda ; X, Y+Z+\Lambda Y Z)\right]\left[1+M F_{p}^{(M)}(\boldsymbol{U}, \Lambda ; Y, Z)\right]
\end{aligned}
$$

that is to say,

$$
\begin{aligned}
F_{p}\left(\alpha^{(M)} \boldsymbol{U}, \Lambda ; X, Y\right) F_{p}\left(\alpha^{(M)} \boldsymbol{U}, \Lambda ; X+Y+\Lambda X Y, Z\right) \\
=F_{p}\left(\alpha^{(M)} \boldsymbol{U}, \Lambda ; X, Y+Z+\Lambda Y Z\right) F_{p}\left(\alpha^{(M)} \boldsymbol{U}, \Lambda ; Y, Z\right) .
\end{aligned}
$$

This is a consequence of $2.5(1)$. The second assertion follows immediately from $2.5(2)$.

Proposition 2.9. Let $\boldsymbol{U}=\left(U_{0}, U_{1}, U_{2}, \ldots\right)$ and $\boldsymbol{V}=\left(V_{0}, V_{1}, V_{2}, \ldots\right)$. Then

$$
\begin{aligned}
F_{p}^{(M)}\left(\boldsymbol{U}+{ }^{(M)} \boldsymbol{V}, \Lambda ; X, Y\right)= & F_{p}^{(M)}(\boldsymbol{U}, \Lambda ; X, Y)+F_{p}^{(M)}(\boldsymbol{V}, \Lambda ; X, Y) \\
& +M F_{p}^{(M)}(\boldsymbol{U}, \Lambda ; X, Y) F_{p}^{(M)}(\boldsymbol{V}, \Lambda ; X, Y)
\end{aligned}
$$

Proof. It is sufficient to prove that

$$
\begin{array}{r}
{\left[1+M F_{p}^{(M)}(\boldsymbol{U}, \Lambda ; X, Y)\right]\left[1+M F_{p}^{(M)}(\boldsymbol{V}, \Lambda ; X, Y)\right]} \\
=1+M F_{p}^{(M)}\left(\boldsymbol{U}+{ }^{(M)} \boldsymbol{V}, \Lambda ; X, Y\right)
\end{array}
$$

that is to say,

$$
F_{p}\left(\alpha^{(M)} \boldsymbol{U}, \Lambda ; X, Y\right) F_{p}\left(\alpha^{(M)} \boldsymbol{V}, \Lambda ; X, Y\right)=F_{p}\left(\alpha^{(M)}\left(\boldsymbol{U}+{ }^{(M)} \boldsymbol{V}\right), \Lambda ; X, Y\right) .
$$

This is a consequence of $2.5(3)$. 


\section{Statement and proof of the theorem}

3.1. Let $A$ be a $\mathbb{Z}[\Lambda]$-algebra. We define a group scheme $\mathcal{G}_{A}^{(\Lambda)}$ over $A$ by

$$
\mathcal{G}_{A}^{(\Lambda)}=\operatorname{Spec} A\left[T, \frac{1}{1+\Lambda T}\right]
$$

with

(1) the multiplication: $T \mapsto T \otimes 1+1 \otimes T+\Lambda T \otimes T$;

(2) the unit: $T \mapsto 0$;

(3) the inverse: $T \mapsto-T /(1+\Lambda T)$.

Moreover, we define an $A$-homomorphism $\alpha_{A}^{(\Lambda)}: \mathcal{G}_{A}^{(\Lambda)} \rightarrow \mathbb{G}_{m, A}$ by

$$
U \mapsto 1+\Lambda T: A\left[U, U^{-1}\right] \rightarrow A\left[T, \frac{1}{1+\Lambda T}\right]
$$

If $\Lambda$ is invertible in $A, \alpha_{A}^{(\Lambda)}$ is an $A$-isomorphism. On the other hand, if $\Lambda=0$ in $A, \mathcal{G}_{A}^{(\Lambda)}$ is nothing but the additive group $\mathbb{G}_{a, A}$.

REMARK 3.2. Let $B=A[t] /\left(t^{2}-\Lambda t\right)$, and let $\varepsilon$ denote the image of $t$ in $B$. Then we have $\varepsilon^{2}=\Lambda \varepsilon$. Defining a ring homomorphism $B \rightarrow A$ by $\varepsilon \mapsto 0$, we have

$$
\operatorname{Ker}\left[B^{\times} \rightarrow A^{\times}\right]=\{1+\varepsilon a ; a \in A, 1+\Lambda a \text { is invertible in } A\} .
$$

Hence $\mathcal{G}_{A}^{(\Lambda)}$ is isomorphic to $\operatorname{Ker}\left[\prod_{B / A} \mathbb{G}_{m, B} \rightarrow \mathbb{G}_{m, A}\right]$, where $\prod_{B / A}$ denotes the Weil restriction functor. Furthermore, the inclusion $A \rightarrow B$ defines a section of $\prod_{B / A} \mathbb{G}_{m, B} \rightarrow \mathbb{G}_{m, A}$, and therefore, the exact sequence

$$
0 \rightarrow \mathcal{G}_{A}^{(\Lambda)} \rightarrow \prod_{B / A} \mathbb{G}_{m, B} \rightarrow \mathbb{G}_{m, A} \rightarrow 0
$$

splits.

Now we recall some results of [13]. For generalities of Hochschild cohomology, see [2, Ch. II.3 and Ch. III.6].

3.3. Let $A$ be a $\mathbb{Z}_{(p)}[\Lambda]$-algebra. We define a complex

$$
C^{1}\left(\widehat{\mathcal{G}}_{A}^{(\Lambda)}, \widehat{\mathbb{G}}_{m, A}\right) \stackrel{\partial}{\longrightarrow} C^{2}\left(\widehat{\mathcal{G}}_{A}^{(\Lambda)}, \widehat{\mathbb{G}}_{m, A}\right)
$$

by

$$
C^{1}\left(\widehat{\mathcal{G}}_{A}^{(\Lambda)}, \widehat{\mathbb{G}}_{m, A}\right)=\{F(T) \in A[[T]] ; F(T) \equiv 1 \bmod \operatorname{deg} 1\} \subset A[[T]]^{\times}
$$


and $C^{2}\left(\widehat{\mathcal{G}}_{A}^{(\Lambda)}, \widehat{\mathbb{G}}_{m, A}\right)=\{G(X, Y) \in A[[X, Y]] ; G(X, Y) \equiv 1 \bmod \operatorname{deg} 1\} \subset A[[X, Y]]^{\times}$

The boundary map $\partial: C^{1}\left(\widehat{\mathcal{G}}^{(\Lambda)}, \widehat{\mathbb{G}}_{m, A}\right) \rightarrow C^{2}\left(\widehat{\mathcal{G}}^{(\Lambda)}, \widehat{\mathbb{G}}_{m, A}\right)$ is given by

$$
\partial: F(T) \mapsto \frac{F(X) F(Y)}{F(X+Y+\Lambda X Y)}
$$

A formal power series $G(X, Y) \in C^{2}\left(\widehat{\mathcal{G}}_{A}^{(\Lambda)}, \widehat{\mathbb{G}}_{m, A}\right)$ is called a symmetric 2-cocycle if $G(X, Y)$ satisfies the following functional equations:

(1) $G(X, Y) G(X+Y+\Lambda X Y, Z)=G(X, Y+Z+\Lambda Y Z) G(Y, Z)$,

(2) $G(X, Y)=G(Y, X)$.

We denote by $Z^{2}\left(\mathcal{G}^{(\Lambda)}, \widehat{\mathbb{G}}_{m, A}\right)$ the subgroup of $C^{2}\left(\widehat{\mathcal{G}}_{A}^{(\Lambda)}, \widehat{\mathbb{G}}_{m, A}\right)$, formed by the symmetric 2-cocycles. $\operatorname{Im} \partial$ is denoted by $B^{2}\left(\widehat{\mathcal{G}}_{A}^{(\Lambda)}, \widehat{\mathbb{G}}_{m, A}\right)$ and we put

$$
H_{0}^{2}\left(\widehat{\mathcal{G}}_{A}^{(\Lambda)}, \widehat{\mathbb{G}}_{m, A}\right)=Z^{2}\left(\widehat{\mathcal{G}}_{A}^{(\Lambda)}, \widehat{\mathbb{G}}_{m, A}\right) / B^{2}\left(\widehat{\mathcal{G}}_{A}^{(\Lambda)}, \widehat{\mathbb{G}}_{m, A}\right)
$$

By abbreviation we denote by $\tilde{C}^{*}\left(\widehat{\mathcal{G}}_{A}^{(\Lambda)}, \widehat{\mathbb{G}}_{m, A}\right)$ the cochain complex

$$
C^{1}\left(\widehat{\mathcal{G}}_{A}^{(\Lambda)}, \widehat{\mathbb{G}}_{m, A}\right) \stackrel{\partial}{\longrightarrow} Z^{2}\left(\widehat{\mathcal{G}}_{A}^{(\Lambda)}, \widehat{\mathbb{G}}_{m, A}\right),
$$

concentrated in the degrees 1 and 2 . We define also a cochain complex

$$
W(A) \stackrel{F-\left[\Lambda^{p-1}\right]}{\longrightarrow} W(A)
$$

denoted by $\tilde{D}^{*}\left(\widehat{\mathcal{G}}_{A}^{(\Lambda)}, \widehat{\mathbb{G}}_{m, A}\right)$. We defined a morphism of complexes

$$
\xi: \tilde{D}^{*}\left(\widehat{\mathcal{G}}_{A}^{(\Lambda)}, \widehat{\mathbb{G}}_{m, A}\right) \rightarrow \tilde{C}^{*}\left(\widehat{\mathcal{G}}_{A}^{(\Lambda)}, \widehat{\mathbb{G}}_{m, A}\right)
$$

by

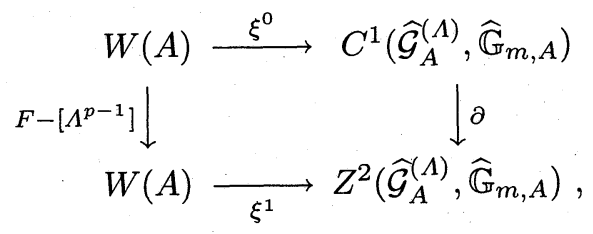

where $\xi^{0}$ is given by

$$
\boldsymbol{a} \mapsto E_{p}(\boldsymbol{a}, \Lambda ; T): W(A) \rightarrow C^{1}\left(\widehat{\mathcal{G}}_{A}^{(\Lambda)}, \widehat{\mathbb{G}}_{m, A}\right) \subset A[[T]]^{\times}
$$


and $\xi^{1}$ by

$$
\boldsymbol{a} \mapsto F_{p}(\boldsymbol{a}, \Lambda ; X, Y): W(A) \rightarrow Z^{2}\left(\widehat{\mathcal{G}}_{A}^{(\Lambda)}, \widehat{\mathbb{G}}_{m, A}\right) \subset A[[X, Y]]^{\times}
$$

$([13,2.19])$

We proved that $\xi: \tilde{D}^{*}\left(\widehat{\mathcal{G}}_{A}^{(\Lambda)}, \widehat{\mathbb{G}}_{m, A}\right) \rightarrow \tilde{C}^{*}\left(\widehat{\mathcal{G}}_{A}^{(\Lambda)}, \widehat{\mathbb{G}}_{m, A}\right)$ induces isomorphism

$$
\xi^{0}: \operatorname{Ker}\left[F-\left[\Lambda^{p-1}\right]: W(A) \rightarrow W(A)\right] \stackrel{\sim}{\rightarrow} \operatorname{Hom}_{A-\mathrm{gr}}\left(\widehat{\mathcal{G}}_{A}^{(\Lambda)}, \widehat{\mathbb{G}}_{m, A}\right)
$$

and

$$
\xi^{1}: \operatorname{Coker}\left[F-\left[\Lambda^{p-1}\right]: W(A) \rightarrow W(A)\right] \stackrel{\sim}{\rightarrow} H_{0}^{2}\left(\widehat{\mathcal{G}}_{A}^{(\Lambda)}, \widehat{\mathbb{G}}_{m, A}\right)
$$

([13, Th. 2.19.1]).

REMARK 3.3.1. In $[13,2.2]$, we defined a complex

$$
C^{1}\left(\widehat{\mathcal{G}}_{A}^{(\Lambda)}, \widehat{\mathbb{G}}_{m, A}\right) \stackrel{\partial}{\longrightarrow} C^{2}\left(\widehat{\mathcal{G}}_{A}^{(\Lambda)}, \widehat{\mathbb{G}}_{m, A}\right)
$$

by

$$
C^{1}\left(\widehat{\mathcal{G}}_{A}^{(\Lambda)}, \widehat{\mathbb{G}}_{m, A}\right)=A[[T]]^{\times}
$$

and

$$
C^{2}\left(\widehat{\mathcal{G}}_{A}^{(\Lambda)}, \widehat{\mathbb{G}}_{m, A}\right)=A[[X, Y]]^{\times}
$$

with the boundary map

$$
\partial: F(T) \mapsto \frac{F(X) F(Y)}{F(X+Y+\Lambda X Y)}
$$

It is readily seen that the inclusions

$$
\{F(T) \in A[[T]] ; F(T) \equiv 1 \bmod \operatorname{deg} 1\} \subset A[[T]]^{\times}
$$

and

$$
\{G(X, Y) \in A[[X, Y]] ; G(X, Y) \equiv 1 \bmod \operatorname{deg} 1\} \subset A[[X, Y]]^{\times}
$$

induce a quasi-isomorphism of the complex defined in 3.3 to the complex defined in $[13,2.2]$.

3.4. Let $A$ be a $\mathbb{Z}_{(p)}[\Lambda, M]$-algebra. We shall define a complex

$$
C^{1}\left(\widehat{\mathcal{G}}_{A}^{(\Lambda)}, \widehat{\mathcal{G}}_{A}^{(M)}\right) \stackrel{\partial}{\longrightarrow} C^{2}\left(\widehat{\mathcal{G}}_{A}^{(\Lambda)}, \widehat{\mathcal{G}}_{A}^{(M)}\right)
$$

by

$$
C^{1}\left(\widehat{\mathcal{G}}_{A}^{(\Lambda)}, \widehat{\mathcal{G}}_{A}^{(M)}\right)=\{F(T) \in A[[T]] ; F(T) \equiv 0 \bmod \operatorname{deg} 1\}
$$


and

$$
C^{2}\left(\widehat{\mathcal{G}}_{A}^{(\Lambda)}, \widehat{\mathcal{G}}_{A}^{(M)}\right)=\{G(X, Y) \in A[[X, Y]] ; G(X, Y) \equiv 0 \bmod \operatorname{deg} 1\}
$$

The group laws of $C^{1}\left(\widehat{\mathcal{G}}_{A}^{(\Lambda)}, \widehat{\mathcal{G}}_{A}^{(M)}\right)$ and $C^{2}\left(\widehat{\mathcal{G}}_{A}^{(\Lambda)}, \widehat{\mathcal{G}}_{A}^{(M)}\right)$ are given by

$$
\left(F_{1}(T), F_{2}(T)\right) \mapsto F_{1}(T)+F_{2}(T)+M F_{1}(T) F_{2}(T)
$$

and

$$
\left(G_{1}(X, Y), G_{2}(X, Y)\right) \mapsto G_{1}(X, Y)+G_{2}(X, Y)+M G_{1}(X, Y) G_{2}(X, Y)
$$

respectively. Moreover, the boundary map $\partial: C^{1}\left(\widehat{\mathcal{G}}_{A}^{(\Lambda)}, \widehat{\mathcal{G}}_{A}^{(M)}\right) \rightarrow C^{2}\left(\widehat{\mathcal{G}}_{A}^{(\Lambda)}, \widehat{\mathcal{G}}_{A}^{(M)}\right)$ is defined by

$$
\partial: F(T) \mapsto \frac{F(X)+F(Y)+M F(X) F(Y)-F(X+Y+\Lambda X Y)}{1+M F(X+Y+\Lambda X Y)} .
$$

A formal power series $G(X, Y) \in C^{2}\left(\widehat{\mathcal{G}}_{A}^{(\Lambda)}, \widehat{\mathcal{G}}_{A}^{(M)}\right)$ is called a symmetric 2-cocycle if $G(X, Y)$ satisfies the following functional equations:

(1) $G(X, Y)+G(X+Y+\Lambda X Y, Z)+M G(X, Y) G(X+Y+\Lambda X Y, Z)$

$$
=G(X, Y+Z+\Lambda Y Z)+G(Y, Z)+M G(X, Y+Z+\Lambda Y Z) G(Y, Z) \text {, }
$$

(2) $G(X, Y)=G(Y, X)$.

For example, $F_{p}^{(M)}(a, \Lambda ; X, Y)$ is a symmetric 2-cocyle, as is shown in Proposition 2.8. We denote by $Z^{2}\left(\mathcal{G}_{A}^{(\Lambda)}, \widehat{\mathcal{G}}_{A}^{(M)}\right)$ the subgroup of $C^{2}\left(\widehat{\mathcal{G}}_{A}^{(\Lambda)}, \widehat{\mathcal{G}}_{A}^{(M)}\right)$, formed by the symmetric 2-cocycles. $\operatorname{Im} \partial$ is denoted by $B^{2}\left(\widehat{\mathcal{G}}_{A}^{(\Lambda)}, \widehat{\mathcal{G}}_{A}^{(M)}\right)$ and we put

$$
H_{0}^{2}\left(\widehat{\mathcal{G}}_{A}^{(\Lambda)}, \widehat{\mathcal{G}}_{A}^{(M)}\right)=Z^{2}\left(\widehat{\mathcal{G}}_{A}^{(\Lambda)}, \widehat{\mathcal{G}}_{A}^{(M)}\right) / B^{2}\left(\widehat{\mathcal{G}}_{A}^{(\Lambda)}, \widehat{\mathcal{G}}_{A}^{(M)}\right)
$$

Again by abbreviation we denote by $\tilde{C}^{*}\left(\widehat{\mathcal{G}}_{A}^{(\Lambda)}, \widehat{\mathcal{G}}_{A}^{(M)}\right)$ the cochain complex

$$
0 \longrightarrow C^{1}\left(\widehat{\mathcal{G}}_{A}^{(\Lambda)}, \widehat{\mathcal{G}}_{A}^{(M)}\right) \stackrel{\partial}{\longrightarrow} Z^{2}\left(\widehat{\mathcal{G}}_{A}^{(\Lambda)}, \widehat{\mathcal{G}}_{A}^{(M)}\right) \longrightarrow 0
$$

concentrated in the degrees 1 and 2 .

3.5. Let $A$ be a $\mathbb{Z}[\Lambda, M]$-algebra and $B=A[t] /\left(t^{2}-M t\right)$. Let $\varepsilon$ denote the image of $t$ in $B$. Then we have $\varepsilon^{2}=M \varepsilon$. The splitting exact sequnce of formal groups

$$
0 \rightarrow \widehat{\mathcal{G}}_{A}^{(M)} \rightarrow\left(\widehat{\prod_{B / A} \mathbb{G}_{m, B}}\right) \rightarrow \widehat{\mathbb{G}}_{m, A} \rightarrow 0
$$


induces a splitting exact sequence of complexes

$$
0 \rightarrow \tilde{C}^{*}\left(\widehat{\mathcal{G}}_{A}^{(\Lambda)}, \widehat{\mathcal{G}}_{A}^{(M)}\right) \rightarrow \tilde{C}^{*}\left(\widehat{\mathcal{G}}_{B}^{(\Lambda)}, \widehat{\mathbb{G}}_{m, B}\right) \rightarrow \tilde{C}^{*}\left(\widehat{\mathcal{G}}_{A}^{(\Lambda)}, \widehat{\mathbb{G}}_{m, A}\right) \rightarrow 0
$$

More precisely,

$$
C^{1}\left(\widehat{\mathcal{G}}_{B}^{(\Lambda)}, \widehat{\mathbb{G}}_{m, B}\right) \rightarrow C^{1}\left(\widehat{\mathcal{G}}_{A}^{(\Lambda)}, \widehat{\mathbb{G}}_{m, A}\right)
$$

and

$$
Z^{2}\left(\widehat{\mathcal{G}}_{B}^{(\Lambda)}, \widehat{\mathbb{G}}_{m, B}\right) \rightarrow Z^{2}\left(\widehat{\mathcal{G}}_{A}^{(\Lambda)}, \widehat{\mathbb{G}}_{m, A}\right)
$$

are induced from the ring homomorphism $B \rightarrow A$. Moreover

$$
C^{1}\left(\widehat{\mathcal{G}}_{A}^{(\Lambda)}, \widehat{\mathcal{G}}_{A}^{(M)}\right) \rightarrow C^{1}\left(\widehat{\mathcal{G}}_{B}^{(\Lambda)}, \widehat{\mathbb{G}}_{m, B}\right)
$$

and

$$
Z^{2}\left(\widehat{\mathcal{G}}_{A}^{(\Lambda)}, \widehat{\mathcal{G}}_{A}^{(M)}\right) \rightarrow Z^{2}\left(\widehat{\mathcal{G}}_{B}^{(\Lambda)}, \widehat{\mathbb{G}}_{m, B}\right)
$$

are defined by

$$
F(T) \mapsto 1+\varepsilon F(T): A[[T]] \rightarrow B[[T]]^{\times}
$$

and

$$
G(X, Y) \mapsto 1+\varepsilon G(X, Y): A[[X, Y]] \rightarrow B[[X, Y]]^{\times}
$$

respectively.

On the other hand, we have a commutative diagram with splitting exact rows

$$
\begin{aligned}
& 0 \longrightarrow W^{(M)}(A) \longrightarrow W(B) \longrightarrow W(A) \longrightarrow 0 \\
& \downarrow F^{(M)}-\left[\Lambda^{p-1}\right] \quad \downarrow F-\left[\Lambda^{p-1}\right] \quad \downarrow F-\left[\Lambda^{p-1}\right] \\
& 0 \longrightarrow W^{(M)}(A) \longrightarrow W(B) \longrightarrow W(A) \longrightarrow 0 \text {, }
\end{aligned}
$$

by Theorem 1.21. Obviously the diagram of complexes

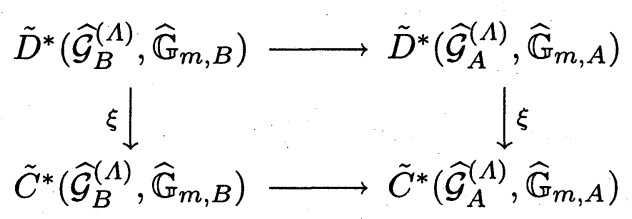

is commutative. Hence we obtain a morphism of complexes

$$
\xi: \tilde{D}^{*}\left(\widehat{\mathcal{G}}_{A}^{(\Lambda)}, \widehat{\mathcal{G}}_{A}^{(M)}\right) \rightarrow \tilde{C}^{*}\left(\widehat{\mathcal{G}}_{A}^{(\Lambda)}, \widehat{\mathcal{G}}_{A}^{(M)}\right)
$$

where $\tilde{D}^{*}\left(\widehat{\mathcal{G}}_{A}^{(\Lambda)}, \widehat{\mathcal{G}}_{A}^{(M)}\right)$ denote the cochain complex

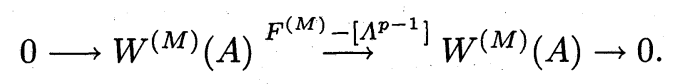


To sum up, we obtain a commutative diagram of cochain complexes with splitting exact rows

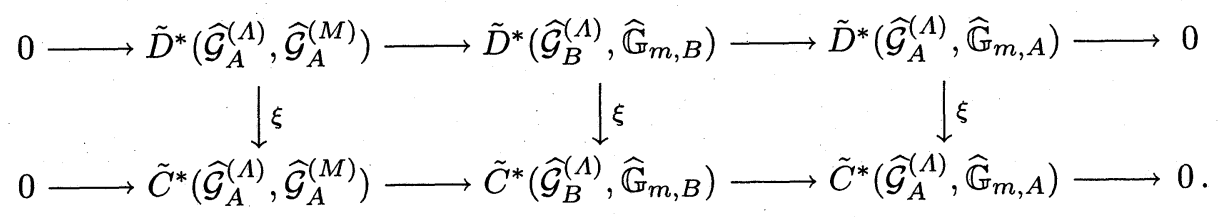

Hence we have the following theorem, noting that

$\xi: \tilde{D}^{*}\left(\widehat{\mathcal{G}}_{B}^{(\Lambda)}, \widehat{\mathbb{G}}_{m, B}\right) \rightarrow \tilde{C}^{*}\left(\widehat{\mathcal{G}}_{B}^{(\Lambda)}, \widehat{\mathbb{G}}_{m, B}\right)$ and $\xi: \tilde{D}^{*}\left(\widehat{\mathcal{G}}_{A}^{(\Lambda)}, \widehat{\mathbb{G}}_{m, A}\right) \rightarrow \tilde{C}^{*}\left(\widehat{\mathcal{G}}_{A}^{(\Lambda)}, \widehat{\mathbb{G}}_{m, A}\right)$ are quasi-isomomorphisms ([13, Th. 2.19.1]).

Theorem 3.5.1. Let $A$ be $\mathbb{Z}_{(p)}[\Lambda, M]$-algebra. Then there exist isomorphisms

$$
\operatorname{Ker}\left[F^{(M)}-\left[\Lambda^{p-1}\right]: W^{(M)}(A) \rightarrow W^{(M)}(A)\right] \stackrel{\sim}{\longrightarrow} \operatorname{Hom}_{A-\mathrm{gr}}\left(\widehat{\mathcal{G}}_{A}^{(\Lambda)}, \widehat{\mathcal{G}}_{A}^{(M)}\right)
$$

and

$$
\operatorname{Coker}\left[F^{(M)}-\left[\Lambda^{p-1}\right]: W^{(M)}(A) \rightarrow W^{(M)}(A)\right] \stackrel{\sim}{\longrightarrow} H_{0}^{2}\left(\widehat{\mathcal{G}}_{A}^{(\Lambda)}, \widehat{\mathcal{G}}_{A}^{(M)}\right)
$$

REMARK 3.6. We can describe explicitly the isomorphisms

$$
\xi^{0}: \operatorname{Ker}\left[F^{(M)}-\left[\Lambda^{p-1}\right]: W^{(M)}(A) \rightarrow W^{(M)}(A)\right] \stackrel{\sim}{\longrightarrow} \operatorname{Hom}_{A-\mathrm{gr}}\left(\widehat{\mathcal{G}}_{A}^{(\Lambda)}, \widehat{\mathcal{G}}_{A}^{(M)}\right)
$$

and

$$
\xi^{1}: \operatorname{Coker}\left[F^{(M)}-\left[\lambda^{p-1}\right]: W^{(M)}(A) \rightarrow W^{(M)}(A)\right] \stackrel{\sim}{\longrightarrow} H_{0}^{2}\left(\widehat{\mathcal{G}}_{A}^{(\Lambda)}, \widehat{\mathcal{G}}_{A}^{(M)}\right)
$$

induced from

$$
\xi: \tilde{D}^{*}\left(\widehat{\mathcal{G}}_{A}^{(\Lambda)}, \widehat{\mathcal{G}}_{A}^{(M)}\right) \rightarrow \tilde{C}^{*}\left(\widehat{\mathcal{G}}_{A}^{(\Lambda)}, \widehat{\mathcal{G}}_{A}^{(M)}\right)
$$

Indeed,

(1) $\boldsymbol{a} \mapsto E_{p}^{(M)}(\boldsymbol{a}, \Lambda ; T)$ gives rise to an isomorphism

$$
\xi^{0}: \operatorname{Ker}\left[F^{(M)}-\left[\Lambda^{p-1}\right]: W^{(M)}(A) \rightarrow W^{(M)}(A)\right] \stackrel{\sim}{\longrightarrow} \operatorname{Hom}_{A-\mathrm{gr}}\left(\widehat{\mathcal{G}}_{A}^{(\Lambda)}, \widehat{\mathcal{G}}_{A}^{(M)}\right) ;
$$

(2) $\boldsymbol{a} \mapsto F_{p}^{(M)}(\boldsymbol{a}, \Lambda ; X, Y)$ gives rise to an isomorphism

$$
\xi^{1}: \operatorname{Coker}\left[F^{(M)}-\left[\Lambda^{p-1}\right]: W^{(M)}(A) \rightarrow W^{(M)}(A)\right] \stackrel{\sim}{\longrightarrow} H_{0}^{2}\left(\widehat{\mathcal{G}}_{A}^{(\Lambda)}, \widehat{\mathcal{G}}_{A}^{(M)}\right) .
$$

This is a direct consequence of the following lemma.

Lemma 3.7. Let $A$ be $a \mathbb{Z}_{(p)}[\Lambda, M]$-algebra and $B=A[t] /\left(t^{2}-M t\right)$, and let $\varepsilon$ denote the image of $t$ in $B$. Let $\boldsymbol{a} \in W^{(M)}(A)$, and put $\varepsilon \boldsymbol{a}=\left(\varepsilon a_{0}, \varepsilon a_{1}, \varepsilon a_{2}, \ldots\right)$. Then: 
(1) $E_{p}(\varepsilon \boldsymbol{a}, \Lambda ; T)=1+\varepsilon E_{p}^{(M)}(\boldsymbol{a}, \Lambda ; T)$;

(2) $F_{p}(\varepsilon \boldsymbol{a}, \Lambda ; X, Y)=1+\varepsilon F_{p}^{(M)}(\boldsymbol{a}, \Lambda ; X, Y)$.

Proof. We may assume that $A=\mathbb{Z}_{(p)}[\Lambda, M]\left[U_{0}, U_{1}, U_{2}, \ldots\right]$ and $\boldsymbol{a}=\boldsymbol{U}=$ $\left(U_{0}, U_{1}, U_{2}, \ldots\right)$. Put

$$
E_{p}(\boldsymbol{U}, \Lambda ; T)=1+\sum_{k=1}^{\infty} a_{k}(\boldsymbol{U}) T^{k}
$$

where $a_{k}(\boldsymbol{U}) \in \mathbb{Z}_{(p)}[\Lambda]\left[U_{0}, U_{1}, U_{2}, \ldots\right]$. Put

$$
a_{k}^{(M)}(\boldsymbol{U})=\frac{1}{M} a_{k}\left(M U_{0}, M U_{1}, M U_{2}, \ldots\right)
$$

Then $a_{k}^{(M)}(\boldsymbol{U}) \in \mathbb{Z}_{(p)}[\Lambda, M]\left[U_{0}, U_{1}, U_{2}, \ldots\right]$ since $a_{k}(\boldsymbol{U})$ has no constant term. Furthermore

$$
E_{p}^{(M)}(\boldsymbol{U}, \Lambda ; T)=\sum_{k=1}^{\infty} a_{k}^{(M)}(\boldsymbol{U}) T^{k}
$$

Now by Lemma 1.20 we have

$$
\varepsilon a_{k}^{(M)}(\boldsymbol{U})=a_{k}\left(\varepsilon U_{0}, \varepsilon U_{1}, \varepsilon U_{2}, \ldots\right)
$$

for $k>0$. This implies that

$$
E_{p}(\varepsilon \boldsymbol{a}, \Lambda ; T)=1+\varepsilon E_{p}^{(M)}(\boldsymbol{a}, \Lambda ; T) .
$$

We can prove (2) similarly.

Example 3.8. Let $A=\mathbb{Z}[M, \Lambda / M]$. Then

$$
\left[\frac{\Lambda}{M}\right]_{M} \in \operatorname{Ker}\left[F^{(M)}-\left[\Lambda^{p-1}\right]: W^{(M)}(A) \rightarrow W^{(M)}(A)\right]
$$

and

$$
E_{p}^{(M)}\left(\left[\frac{\Lambda}{M}\right]_{M}, \Lambda ; T\right)=\frac{\Lambda}{M} T .
$$

(Cf. Example 2.2.1 and 2.2.2.)

Example 3.9 . Put

$$
p^{n}[1]=\left(q_{0}, q_{1}, q_{2}, \ldots\right) \in W(\mathbb{Z}) .
$$

Then, by Lemma 1.13.1,

$$
p^{n}[1]_{\Lambda}=\left(q_{0}, q_{1} \Lambda^{p-1}, q_{2} \Lambda^{p^{2}-1}, \ldots\right) \in W^{(\Lambda)}(\mathbb{Z}[\Lambda]) .
$$


Let

$$
A=\mathbb{Z}\left[\Lambda, M, \frac{p^{n} \Lambda}{M}, \frac{p^{n-1} \Lambda^{p}}{M}, \ldots, \frac{\Lambda^{p^{n}}}{M}\right]
$$

and put

$$
\boldsymbol{q}=p^{n}\left[\frac{\Lambda}{M}\right]_{M}=\left(\frac{q_{0} \Lambda}{M}, \frac{q_{1} \Lambda^{p}}{M}, \frac{q_{2} \Lambda^{p^{2}}}{M}, \ldots\right) \in W^{(M)}(A)
$$

Then

$$
\boldsymbol{q} \in \operatorname{Ker}\left[F^{(M)}-\left[\Lambda^{p-1}\right]: W^{(M)}(A) \rightarrow W^{(M)}(A)\right]
$$

and

$$
E_{p}^{(M)}(\boldsymbol{q}, \Lambda ; T)=\frac{(1+\Lambda T)^{p^{n}}-1}{M} .
$$

Example 3.10 . Let $A$ be a $\mathbb{Z}_{(p)}[\Lambda]$-algebra. As is remarked in 1.9 .2 , the additive group $W^{(0)}(A)$ is isomorphic to the direct product $A^{\mathbb{N}}$ and $F^{(0)}-\left[\Lambda^{p-1}\right]$ : $W^{(0)}(A) \rightarrow W^{(0)}(A)$ is given by

$$
\Phi:\left(a_{0}, a_{1}, a_{2}, \ldots\right) \mapsto\left(p a_{1}-\Lambda^{p-1} a_{0}, p a_{2}-\Lambda^{p(p-1)} a_{1}, p a_{3}-\Lambda^{p^{2}(p-1)} a_{2}, \ldots\right) .
$$

Then:

(1) $\xi^{0}: \boldsymbol{a} \mapsto E_{p}^{(0)}(\boldsymbol{a}, \Lambda ; T)$ gives rise to an isomorphism

$$
\operatorname{Ker}\left[\Phi: A^{\mathbb{N}} \rightarrow A^{\mathbb{N}}\right] \stackrel{\sim}{\longrightarrow} \operatorname{Hom}_{A-\mathrm{gr}}\left(\widehat{\mathcal{G}}_{A}^{(\Lambda)}, \widehat{\mathbb{G}}_{a, A}\right) ;
$$

(2) $\xi: \boldsymbol{a} \mapsto F_{p}^{(0)}(\boldsymbol{a}, \Lambda ; X, Y)$ gives rise to an isomorphism

$$
\operatorname{Coker}\left[\Phi: A^{\mathbb{N}} \rightarrow A^{\mathbb{N}}\right] \stackrel{\sim}{\longrightarrow} H_{0}^{2}\left(\widehat{\mathcal{G}}_{A}^{(\Lambda)}, \widehat{\mathbb{G}}_{a, A}\right),
$$

as was mentioned in Yato [16].

Assume now that $A$ is a $\mathbb{Z}_{(p)}\left[\Lambda, \Lambda^{p-1} / p\right]$-algebra. Let $a \in A$, and put

$$
\boldsymbol{a}=\left(a, \frac{\Lambda^{p-1}}{p} a, \frac{\Lambda^{p^{2}-1}}{p^{2}} a, \frac{\Lambda^{p^{3}-1}}{p^{3}} a, \ldots\right) \in A^{\mathbb{N}} .
$$

Then $\boldsymbol{a} \in \operatorname{Ker}\left[\Phi: A^{\mathbb{N}} \rightarrow A^{\mathbb{N}}\right]$, and we have

$$
E_{p}^{(0)}(a, \Lambda ; T)=\frac{a}{\Lambda} \log (1+\Lambda T)
$$

EXAMPLE 3.11. Let $A$ be a $\mathbb{Z}_{(p)}[\Lambda, M]$-algebra. The homomorphism of formal groups $\alpha^{(M)}: \widehat{\mathcal{G}}_{A}^{(M)} \rightarrow \widehat{\mathbb{G}}_{m, A}$ induces a morphism of cochain complexes

$$
\alpha^{(M)}: \tilde{C}^{*}\left(\widehat{\mathcal{G}}_{A}^{(\Lambda)}, \widehat{\mathcal{G}}_{A}^{(M)}\right) \rightarrow \tilde{C}^{*}\left(\widehat{\mathcal{G}}_{A}^{(\Lambda)}, \widehat{\mathbb{G}}_{m, A}\right) .
$$


We can easily verify the commutativity of the diagrams

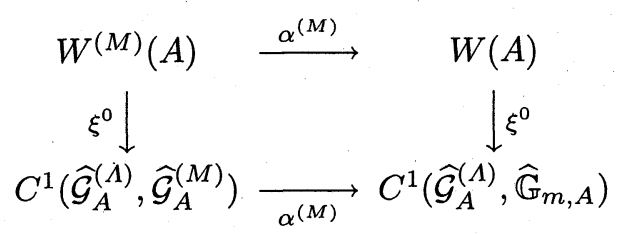

and

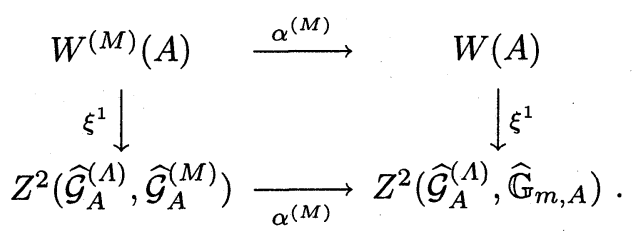

Moreover, we obtain a commutative diagram of cochain complexes

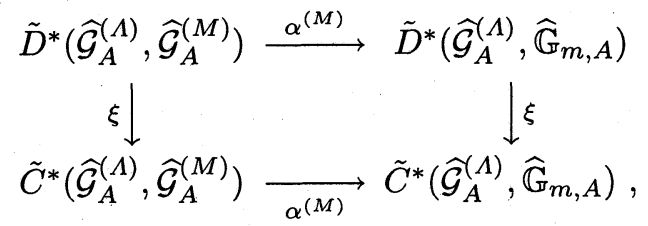

and therefore commutative diagrams

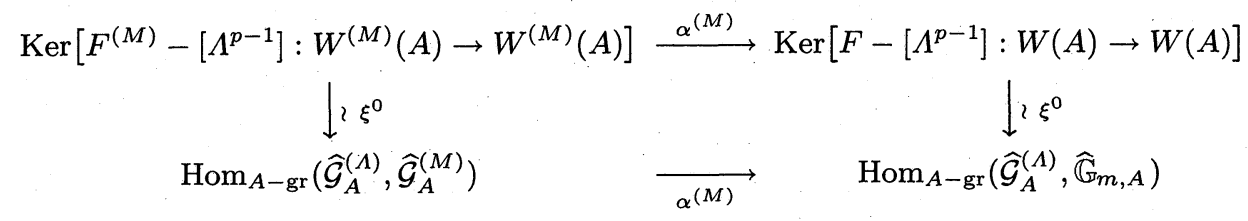

and
$\operatorname{Coker}\left[F^{(M)}-\left[\Lambda^{p-1}\right]: W^{(M)}(A) \rightarrow W^{(M)}(A)\right] \stackrel{\alpha^{(M)}}{\longrightarrow} \operatorname{Coker}\left[F-\left[\Lambda^{p-1}\right]: W(A) \rightarrow W(A)\right]$ $\downarrow \imath \xi^{1} \quad\left\lfloor\imath \xi^{1}\right.$ $H_{0}^{2}\left(\widehat{\mathcal{G}}_{A}^{(\Lambda)}, \widehat{\mathcal{G}}_{A}^{(M)}\right) \quad \underset{\alpha(M)}{\longrightarrow} \quad H_{0}^{2}\left(\widehat{\mathcal{G}}_{A}^{(\Lambda)}, \widehat{\mathbb{G}}_{m, A}\right)$.

Assume now the homothety by $M$ is not bijective but injective on $A$, and put $A_{0}=A /(M)$. Then we have a commutative diagram of cochain complexes with exact rows

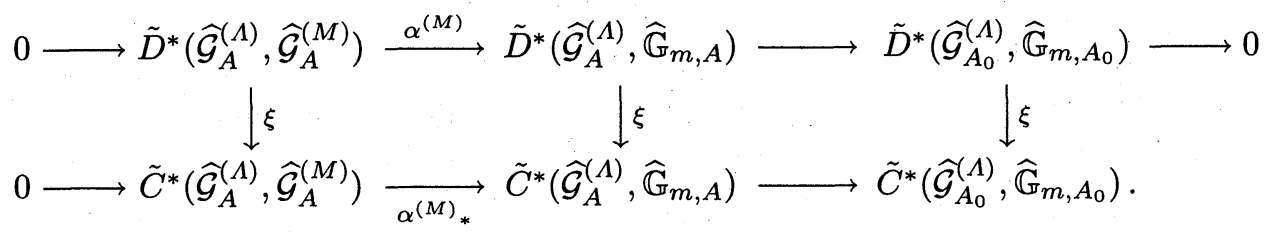


Hence it is readily seen that the exact sequence

$$
\begin{array}{r}
0 \rightarrow \operatorname{Hom}_{A-\mathrm{gr}}\left(\widehat{\mathcal{G}}_{A}^{(\Lambda)}, \widehat{\mathcal{G}}_{A}^{(M)}\right) \rightarrow \operatorname{Hom}_{A-\mathrm{gr}}\left(\widehat{\mathcal{G}}_{A}^{(\Lambda)}, \widehat{\mathbb{G}}_{m, A}\right) \rightarrow \operatorname{Hom}_{A_{0}-\mathrm{gr}}\left(\widehat{\mathcal{G}}_{A_{0}}^{(\Lambda)}, \widehat{\mathbb{G}}_{m, A_{0}}\right) \\
\rightarrow H_{0}^{2}\left(\widehat{\mathcal{G}}_{A}^{(\Lambda)}, \widehat{\mathcal{G}}_{A}^{(M)}\right) \rightarrow H_{0}^{2}\left(\widehat{\mathcal{G}}_{A}^{(\Lambda)}, \widehat{\mathbb{G}}_{m, A}\right) \rightarrow H_{0}^{2}\left(\widehat{\mathcal{G}}_{A_{0}}^{(\Lambda)}, \widehat{\mathbb{G}}_{m, A_{0}}\right) \rightarrow 0
\end{array}
$$

is deduced from the commutative diagram with exact rows

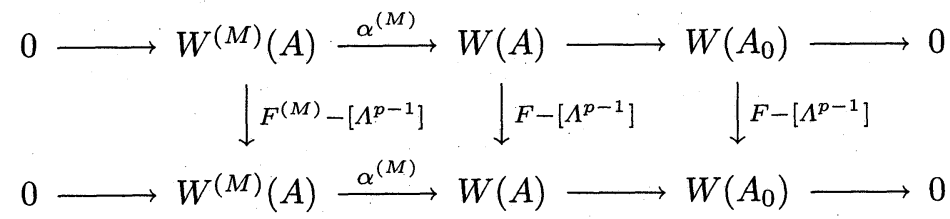

by the snake lemma.

Remark 3.12. Let $A$ be a $\mathbb{Z}_{(p)}[\Lambda]$-algebra. Assume that $\Lambda$ is nilpotent in $A$. We proved that

(1) $\boldsymbol{a} \mapsto E_{p}(\boldsymbol{a}, \Lambda ; T)$ gives rise to an isomorphism

$$
\operatorname{Ker}\left[F-\left[\Lambda^{p-1}\right]: \widehat{W}(A) \rightarrow \widehat{W}(A)\right] \stackrel{\sim}{\longrightarrow} \operatorname{Hom}_{A-\mathrm{gr}}\left(\mathcal{G}_{A}^{(\Lambda)}, \mathbb{G}_{m, A}\right) ;
$$

(2) $\boldsymbol{a} \mapsto F_{p}(\boldsymbol{a}, \Lambda ; X, Y)$ gives rise to an isomorphism

$$
\operatorname{Coker}\left[F-\left[\Lambda^{p-1}\right]: \widehat{W}(A) \rightarrow \widehat{W}(A)\right] \stackrel{\sim}{\longrightarrow} H_{0}^{2}\left(\mathcal{G}_{A}^{(\Lambda)}, \mathbb{G}_{m, A}\right)
$$

([13, Th. 2.7.1]).

Now let $A$ be a $\mathbb{Z}_{(p)}[\Lambda, M]$-algebra. Assume that $\Lambda$ is nilpotent in $A$. We can conclude similarly as in Theorem 3.5.1 that

(1) $\boldsymbol{a} \mapsto E_{p}^{(M)}(\boldsymbol{a}, \Lambda ; T)$ gives rise to an isomorphism

$$
\operatorname{Ker}\left[F^{(M)}-\left[\Lambda^{p-1}\right]: \widehat{W}^{(M)}(A) \rightarrow \widehat{W}^{(M)}(A)\right] \stackrel{\sim}{\longrightarrow} \operatorname{Hom}_{A-\mathrm{gr}}\left(\mathcal{G}_{A}^{(\Lambda)}, \mathcal{G}_{A}^{(M)}\right) ;
$$

(2) $\boldsymbol{a} \mapsto F_{p}^{(M)}(\boldsymbol{a}, \Lambda ; X, Y)$ gives rise to an isomorphism

$$
\operatorname{Coker}\left[F^{(M)}-\left[\Lambda^{p-1}\right]: \widehat{W}^{(M)}(A) \rightarrow \widehat{W}^{(M)}(A)\right] \stackrel{\sim}{\longrightarrow} H_{0}^{2}\left(\mathcal{G}_{A}^{(\Lambda)}, \mathcal{G}_{A}^{(M)}\right) .
$$

Here $\widehat{W}^{(M)}$ denote the functor defined by

$$
\widehat{W}^{(M)}(A)=\left\{\left(a_{0}, a_{1}, a_{2}, \ldots\right) \in W^{(M)}(A) ; \begin{array}{l}
M a_{i} \text { is nilpotent for all } i \text { and } \\
a_{i}=0 \text { for all but a finite number of } i
\end{array}\right\} .
$$


Then we have a splitting exact sequence

$$
0 \rightarrow \widehat{W}^{(M)}(A) \rightarrow \widehat{W}(B) \rightarrow \widehat{W}(A) \rightarrow 0
$$

where $B=A[t] /\left(t^{2}-M t\right)$.

We conclude the section by giving some remarks on a case of extensions over a discrete valuation ring $[6,7,8]$.

Remark 3.13. Let $A$ be a discrete valuation ring and $\mathfrak{m}$ (resp. $k, K$ ) the maximal ideal (resp. the residue field, the field of fractions) of $A$. Take a uniformizing parameter $\pi$ of $A$. We denote by $v$ the valuation of $A$ normalized by $v(\pi)=1$.

Let $\lambda, \mu \in \mathfrak{m}-\{0\}$. Put $n=v(\mu)$ and $A_{0}=A / \mathfrak{m}^{n}$. Let $F(T) \in A[T]$, satisfying

1) $F(0) \equiv 1 \bmod \mu$; 2) $F(X) F(Y) \equiv F(X+Y+\lambda X Y) \bmod \mu$.

We defined a smooth affine group scheme $\mathcal{E}^{(\lambda, \mu ; F)}$ over $A$ by

$$
\mathcal{E}^{(\lambda, \mu ; F)}=\operatorname{Spec} A\left[T_{0}, T_{1}, \frac{1}{1+\lambda T_{0}}, \frac{1}{F\left(T_{0}\right)+\mu T_{1}}\right]
$$

with the multiplication:

$$
\begin{aligned}
& T_{0} \mapsto \lambda T_{0} \otimes T_{0}+T_{0} \otimes 1+1 \otimes T_{0}, \\
& \begin{array}{l}
T_{1} \mapsto \mu T_{1} \otimes T_{1}+T_{1} \otimes F\left(T_{0}\right)+F\left(T_{0}\right) \otimes T_{1} \\
\quad+\frac{1}{\mu}\left[F\left(T_{0}\right) \otimes F\left(T_{0}\right)-F\left(\lambda T_{0} \otimes T_{0}+T_{0} \otimes 1+1 \otimes T_{0}\right)\right]
\end{array}
\end{aligned}
$$

Furthermore we proved that $F \mapsto\left[\mathcal{E}^{(\lambda, \mu ; F)}\right]$ gives rise to a surjective homomorphism

$$
\partial: \operatorname{Hom}_{A_{0}-\operatorname{gr}}\left(\mathcal{G}^{(\lambda)}, \mathbb{G}_{m, A_{0}}\right) \rightarrow \operatorname{Ext}_{A}^{1}\left(\mathcal{G}^{(\lambda)}, \mathcal{G}^{(\mu)}\right)
$$

and Ker $\partial$ is generated by the class of $1+\lambda T$ (cf. [6, Sect. 3] or [8, II.1.2]).

Now note that $F(T)$ is invertible in $A[[T]]$. Then

$$
\left(T_{0}, T_{1}\right) \mapsto\left(T_{0}, \frac{T_{1}}{F\left(T_{0}\right)}\right)
$$

defines an isomorphism of formal groups

$$
\widehat{\mathcal{E}}^{(\lambda, \mu ; F)}=\operatorname{Spf} A\left[\left[T_{0}, T_{1}\right]\right] \stackrel{\sim}{\longrightarrow} \widehat{\mathcal{E}}=\operatorname{Spf} A\left[\left[T_{0}, T_{1}\right]\right]
$$

where $\widehat{\mathcal{E}}$ is the extension of $\widehat{\mathcal{G}}^{(\lambda)}$ by $\widehat{\mathcal{G}}^{(\mu)}$ defined by the 2-cocycle

$$
(\partial F)(X, Y)=\frac{1}{\mu}\left[\frac{F(X) F(Y)}{F(\lambda X Y+X+Y)}-1\right] \in Z^{2}\left(\widehat{\mathcal{G}}^{(\lambda)}, \widehat{\mathcal{G}}^{(\mu)}\right) .
$$


Furthermore, defining a homomorphism

$$
\operatorname{Ext}_{A}^{1}\left(\mathcal{G}^{(\lambda)}, \mathcal{G}^{(\mu)}\right) \rightarrow H_{0}^{2}\left(\widehat{\mathcal{G}}^{(\lambda)}, \widehat{\mathcal{G}}^{(\mu)}\right)
$$

by

$$
\left[\mathcal{E}^{(\lambda, \mu ; F)}\right] \mapsto[(\partial F)(X, Y)]
$$

we obtain a commutative diagram with exact rows

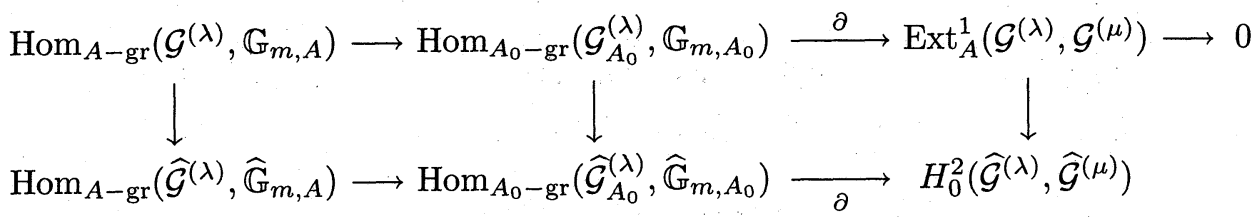

(cf. [6] or [7]).

Assume now that $A$ is of mixed characteristics 0 and $p$. Let $\boldsymbol{a}=$ $\left(a_{0}, a_{1}, a_{2}, \ldots\right) \in \operatorname{Ker}\left[F-\left[\lambda^{p-1}\right]: W(K) \rightarrow W(K)\right]$. Then $a_{r}(r>0)$ is determined inductively by

$$
\Phi_{r}\left(a_{0}, \ldots, a_{r-1}, a_{r}\right)=\lambda^{p^{r-1}(p-1)} \Phi_{r-1}\left(a_{0}, \ldots, a_{r-1}\right),
$$

and we obtain

$$
\Phi_{r}(\boldsymbol{a})=\lambda^{p^{r}-1} a_{0}(r>0)
$$

and

$$
E_{p}(\boldsymbol{a}, \lambda ; T)=(1+\lambda T)^{a_{0} / \lambda} .
$$

Furthermore, $\boldsymbol{a} \in W(A)$ if and only if $(1+\lambda T)^{a_{0} / \lambda} \in A[[T]]$.

Proposition 3.13.1. If $v(\lambda) \geq \frac{v(p)}{p-1} \geq v(\mu)$, then the reduction map

$$
\operatorname{Hom}_{A-\mathrm{gr}}\left(\widehat{\mathcal{G}}^{(\lambda)}, \widehat{\mathbb{G}}_{m, A}\right) \rightarrow \operatorname{Hom}_{A_{0}-\mathrm{gr}}\left(\widehat{\mathcal{G}}_{A_{0}}^{(\lambda)}, \widehat{\mathbb{G}}_{m, A_{0}}\right)
$$

is zero, and therefore, the canonical map

$$
\operatorname{Ext}_{A}^{1}\left(\mathcal{G}^{(\lambda)}, \mathcal{G}^{(\mu)}\right) \rightarrow \operatorname{Ext}_{A}^{1}\left(\widehat{\mathcal{G}}^{(\lambda)}, \widehat{\mathcal{G}}^{(\mu)}\right)
$$

is injective.

Proof. Let $a \in A$, and put $(1+\lambda T)^{a / \lambda}=\sum_{j=0}^{\infty} c_{j} T^{j}$. It is verified without difficulty that, if $v(a) \geq \frac{v(p)}{p-1}$, then $v\left(c_{k}\right) \geq \frac{v(p)}{p-1}$ for $k>0$, which implies that $(1+\lambda T)^{a / \lambda} \in A[[T]]$. Furthermore, $(1+\lambda T)^{a / \lambda} \equiv 1 \bmod \mu$ since $\frac{v(p)}{p-1} \geq v(\mu)$. On the other hand, it is verified that, if $v(a)<\frac{v(p)}{p-1}$, then $\lim _{r \rightarrow \infty} v\left(c_{p^{r}}\right)=-\infty$, which implies that $(1+\lambda T)^{a / \lambda} \notin A[[T]]$. Hence the result. 
EXAMPLE 3.13.2. If $v(\lambda) \geq v(\mu)>\frac{v(p)}{p-1}+1$, then the canonical map

$$
\operatorname{Ext}_{A}^{1}\left(\mathcal{G}^{(\lambda)}, \mathcal{G}^{(\mu)}\right) \rightarrow \operatorname{Ext}_{A}^{1}\left(\widehat{\mathcal{G}}^{(\lambda)}, \widehat{\mathcal{G}}^{(\mu)}\right)
$$

is not injective.

Indeed, take $a \in A$ with $v(\mu)>v(a)>\frac{v(p)}{p-1}$ and put $(1+\lambda T)^{a / \lambda}=\sum_{j=0}^{\infty} c_{j} T^{j}$. Then $(1+\lambda T)^{a / \lambda} \in A[[T]]$ and $\lim _{j \rightarrow \infty} v\left(c_{j}\right)=\infty$ since $v(\lambda)>v(a)>\frac{v(p)}{p-1}$. Therefore, $(1+\lambda T)^{a / \lambda} \bmod \mu \in A_{0}[T]$. On the other hand, $(1+\lambda T)^{a / \lambda} \not \equiv 1 \bmod \mu$ since $v(a)<v(\mu)$.

These imply that

(1) the class $\left[\mathcal{E}^{(\lambda, \mu ; F)}\right]$ is not trivial in $\operatorname{Ext}_{A}^{1}\left(\mathcal{G}^{(\lambda)}, \mathcal{G}^{(\mu)}\right)$;

(2) the image of $\left[\mathcal{E}^{(\lambda, \mu ; F)}\right]$ is trivial in $\operatorname{Ext}_{A}^{1}\left(\widehat{\mathcal{G}}^{(\lambda)}, \widehat{\mathcal{G}}^{(\mu)}\right)$.

Here $F(T)=(1+\lambda T)^{a / \lambda} \bmod \mu \in A_{0}[T]$.

\section{Around the Cartier theory}

4.1. Let $A$ be a ring. Let $R$ denote the ring, generated by $\mathbb{V}$ over $W(A)$, with the relation:

(1) $\boldsymbol{a} \mathbb{V}=\mathbb{V} F(\boldsymbol{a})$

Any element of $R$ is expressed uniquely in the form

$$
\boldsymbol{a}_{0}+\mathbb{V} \boldsymbol{a}_{1}+\cdots+\mathbb{V}^{n} \boldsymbol{a}_{n}, \quad \boldsymbol{a}_{j} \in W(A) .
$$

By the relation (1), $\mathbb{V}^{n} R$ is a two-sided ideal of $R$. Put $\widehat{R}=\underset{n}{\lim } R / \mathbb{V}^{n} R$. Now we denote by $D_{A}$ the ring, generated by $\mathbb{F}$ over $\widehat{R}$, with the relations:

(2) $\mathbb{F} \boldsymbol{a}=F(\boldsymbol{a}) \mathbb{F}$,

(3) $\mathbb{F} \mathbb{V}=p$,

(4) $\mathbb{V} a \mathbb{F}=V(\boldsymbol{a})$

Any element of $D_{A}$ is expressed uniquely in the form

$$
\sum_{j=1}^{m} \boldsymbol{a}_{-j} \mathbb{F}^{j}+\boldsymbol{a}_{0}+\sum_{j=1}^{\infty} \mathbb{V}^{j} \boldsymbol{a}_{j}, \quad \boldsymbol{a}_{j} \in W(A) .
$$

$D_{A}$ is called the Cartier-Dieudonné algebra or the Dieudonné algebra over $A$. (Cf. $[4,28.1]$.)

Example 4.2.1. Let $A$ be a ring. Let $\boldsymbol{a} \in W(A)$. Then

$$
\boldsymbol{T}=\left(T_{0}, T_{1}, T_{2}, \ldots\right) \mapsto \boldsymbol{a} \boldsymbol{T}=\left(P_{0}(\boldsymbol{a}, \boldsymbol{T}), P_{1}(\boldsymbol{a}, \boldsymbol{T}), P_{2}(\boldsymbol{a}, \boldsymbol{T}), \ldots\right)
$$


defines an endomorphism of the formal group $\widehat{W}_{A}$. Moreover,

$$
\boldsymbol{T} \mapsto\left(\sum_{j=1}^{m} V^{j} \boldsymbol{a}_{-j}+\boldsymbol{a}_{0}+\sum_{j=1}^{\infty} \boldsymbol{a}_{j} F^{j}\right) \boldsymbol{T}, \quad \boldsymbol{a}_{j} \in W(A)
$$

defines an endomorphism of $\widehat{W}_{A} . \quad V \mapsto \mathbb{F}$ and $F \mapsto \mathbb{V}$ give rise to an antiisomorphism of $\operatorname{End}_{A-\mathrm{gr}} \widehat{W}_{A}$ to $D_{A}$ (cf. [4, 27.2.12]).

ExAmple 4.2.2. Let $A$ be a ring. $W(A)$ is a left $D_{A}$-module, equipped with $\mathbb{F}=F$ and $\mathbb{V}=V$.

Example 4.2.3. Let $A$ be a $\mathbb{Z}[M]$-algebra. $W^{(M)}(A)$ is a left $D_{A}$-module, equipped with $\mathbb{F}=F^{(M)}$ and $\mathbb{V}=V$. Furthermore, $\alpha^{(M)}: W^{(M)}(A) \rightarrow W(A)$ is a $D_{A}$-homomorphism.

REMARK 4.2.4. Let $A$ be a ring and $\boldsymbol{P}=\sum_{j=0}^{\infty} \mathbb{V}^{j} \boldsymbol{a}_{j} \in D_{A}$. If $\boldsymbol{a}_{0}$ is invertible in $W(A)$, then $\boldsymbol{P}$ is invertible in $D_{A}$.

Indeed, define $\boldsymbol{b}_{k} \in W(A)$ inductively by

$$
b_{0}=a_{0}^{-1}
$$

and

$$
\boldsymbol{b}_{k}=-\left(F^{k} \boldsymbol{a}_{0}\right)^{-1}\left(\sum_{i=1}^{k}\left(F^{k-i} \boldsymbol{a}_{i}\right) \boldsymbol{b}_{k-i}\right) .
$$

Then $\boldsymbol{Q}=\sum_{j=0}^{\infty} \mathbb{V}^{j} \boldsymbol{b}_{j}$ is the inverse of $\boldsymbol{P}=\sum_{j=0}^{\infty} \mathbb{V}^{j} \boldsymbol{a}_{j}$.

4.3. Let $A$ be a $\mathbb{Z}_{(p)}[\Lambda]$-algebra. Define a $D_{A}$-homomorphism $\varphi_{\Lambda}: D_{A} \rightarrow$ $W^{(\Lambda)}(A)$ by

$$
\begin{aligned}
\varphi: & \sum_{j=1}^{m} \boldsymbol{a}_{-j} \mathbb{F}^{j}+\boldsymbol{a}_{0}+\sum_{j=1}^{\infty} \mathbb{V}^{j} \boldsymbol{a}_{j} \mapsto\left(\sum_{j=1}^{m} \boldsymbol{a}_{-j} \mathbb{F}^{j}+\boldsymbol{a}_{0}+\sum_{j=1}^{\infty} \mathbb{V}^{j} \boldsymbol{a}_{j}\right)[1]_{\Lambda} \\
& =\sum_{j=1}^{m} \boldsymbol{a}_{-j} \cdot F^{(\Lambda)^{j}}\left([1]_{\Lambda}\right)+\sum_{j=0}^{\infty} V^{j}\left(\boldsymbol{a}_{j} \cdot[1]_{\Lambda}\right) \\
& =\sum_{j=1}^{m} \boldsymbol{a}_{-j} \cdot\left[\Lambda^{p^{j}-1}\right]_{\Lambda}+\sum_{j=0}^{\infty} V^{j}\left(\boldsymbol{a}_{j} \cdot[1]_{\Lambda}\right) .
\end{aligned}
$$

Since

$$
\varphi_{\Lambda}\left(\mathbb{F}-\left[\Lambda^{p-1}\right]\right)=\left[\Lambda^{p-1}\right]_{\Lambda}-\left[\Lambda^{p-1}\right] \cdot[1]_{\Lambda}=0,
$$

$\varphi_{\Lambda}$ induces a $D_{A}$-homomorphism

$$
\tilde{\varphi}_{\Lambda}: D_{A} / D_{A}\left(\mathbb{F}-\left[\Lambda^{p-1}\right]\right) \rightarrow W^{(\Lambda)}(A) .
$$


Theorem 4.4. Under the notation above, $\tilde{\varphi}_{\Lambda}$ is bijective.

Proof. It is obvious that $\tilde{\varphi}$ is surjective since

$$
\varphi\left(\sum_{j=0}^{\infty} \mathbb{V}^{j}\left[a_{j}\right]\right)=\left(\sum_{j=0}^{\infty} \mathbb{V}^{j}\left[a_{j}\right]\right)[1]_{\Lambda}=\sum_{j=0}^{\infty} V\left(\left[a_{j}\right]_{\Lambda}\right)
$$

Assume that

$$
\varphi\left(\sum_{j=1}^{m} \boldsymbol{a}_{-j} \mathbb{F}^{j}+\boldsymbol{a}_{0}+\sum_{j=1}^{\infty} \mathbb{V}^{j} \boldsymbol{a}_{j}\right)=0 .
$$

Define $\boldsymbol{b}_{-m+1}, \ldots, \boldsymbol{b}_{0}, \boldsymbol{r} \in W(A)$ inductively by

$$
\begin{gathered}
\boldsymbol{b}_{-m+1}=\boldsymbol{a}_{-m}, \quad \boldsymbol{b}_{-m+i}=\boldsymbol{a}_{-m+i-1}+\left[\Lambda^{p^{m-i+1}(p-1)}\right] \boldsymbol{b}_{-m+i-1}(1<i \leq m), \\
\boldsymbol{r}=\boldsymbol{a}_{0}+\left[\Lambda^{p-1}\right] \boldsymbol{b}_{0} .
\end{gathered}
$$

Then we have

$$
\sum_{j=0}^{m} \boldsymbol{a}_{-j} \mathbb{F}^{j}=\left(\sum_{j=0}^{m-1} \boldsymbol{b}_{-j} \mathbb{F}^{j}\right)\left(\mathbb{F}-\left[\Lambda^{p-1}\right]\right)+\boldsymbol{r} .
$$

Therefore we may assume that

$$
\varphi\left(\sum_{j=0}^{\infty} \mathbb{V}^{j} \boldsymbol{a}_{j}\right)=0
$$

Now we verify that there exist $\boldsymbol{b}_{0}, \boldsymbol{b}_{1}, \boldsymbol{b}_{2}, \ldots \in W(A)$ such that $V \boldsymbol{b}_{0}=\boldsymbol{a}_{0}$ and $V \boldsymbol{b}_{j}=\boldsymbol{a}_{j}+\left[\Lambda^{p-1}\right] \boldsymbol{b}_{j-1}$ for each $j>0$.

In fact, since

$$
\left(\sum_{j=0}^{\infty} \mathbb{V}^{j} \boldsymbol{a}_{j}\right)[1]_{\Lambda}=0
$$

$\boldsymbol{a}_{0} \cdot[1]_{\Lambda} \in \operatorname{Im}\left[V: W^{(\Lambda)}(A) \rightarrow W^{(\Lambda)}(A)\right]$, and therefore, $\boldsymbol{a}_{0} \in \operatorname{Im}[V: W(A) \rightarrow$ $W(A)]$. Now assume that there exist $\boldsymbol{b}_{0}, \boldsymbol{b}_{1}, \ldots, \boldsymbol{b}_{n} \in W(A)$ such that $V \boldsymbol{b}_{j}=$ $\boldsymbol{a}_{j}+\left[\Lambda^{p-1}\right] \boldsymbol{b}_{j-1}$ for $0 \leq j \leq n$. Here we set $\boldsymbol{b}_{-1}=\mathbf{0}$. Then we have

$$
\sum_{j=0}^{n} \mathbb{V}^{j} \boldsymbol{a}_{j}=\left(\sum_{j=0}^{n} \mathbb{V}^{j+1} \boldsymbol{b}_{j}\right)\left(\mathbb{F}-\left[\Lambda^{p-1}\right]\right)+\mathbb{V}^{n+1}\left(\left[\Lambda^{p-1}\right] \boldsymbol{b}_{n}\right)
$$

and therefore,

$$
V^{n+1}\left(\left[\Lambda^{p-1}\right] \boldsymbol{b}_{n} \cdot[1]_{\Lambda}\right)+\sum_{j=n+1}^{\infty} V^{j}\left(\boldsymbol{a}_{j} \cdot[1]_{\Lambda}\right)=0
$$


It follows that $V^{n+1}\left(\left(\left[\Lambda^{p-1}\right] \boldsymbol{b}_{n}+\boldsymbol{a}_{n+1}\right) \cdot[1]_{\Lambda}\right) \in \operatorname{Im}\left[V^{n+2}: W^{(\Lambda)}(A) \rightarrow W^{(\Lambda)}(A)\right]$ and that $\left[\Lambda^{p-1}\right] \boldsymbol{b}_{n}+\boldsymbol{a}_{n+1} \in \operatorname{Im}[V: W(A) \rightarrow W(A)]$.

Hence we have obtained

$$
\sum_{j=0}^{\infty} \mathbb{V}^{j} \boldsymbol{a}_{j}=\left(\sum_{j=0}^{\infty} \mathbb{V}^{j+1} \boldsymbol{b}_{j}\right)\left(\mathbb{F}-\left[\Lambda^{p-1}\right]\right)
$$

and we can conclude that $\operatorname{Ker} \varphi=D_{A}\left(\mathbb{F}-\left[\Lambda^{p-1}\right]\right)$ and that $\tilde{\varphi}_{\Lambda}$ is injective.

Corollary 4.5. The sequence $0 \rightarrow D_{A} \stackrel{\cdot\left(\mathbb{F}-\left[\Lambda^{p-1}\right]\right)}{\longrightarrow} D_{A} \stackrel{\varphi_{\Lambda}}{\longrightarrow} W^{(\Lambda)}(A) \rightarrow 0$ is a free resolution of $W^{(\Lambda)}(A)$.

Corollary 4.6. Let $E$ be a left $D_{A}$-module. Then:

(1) $\operatorname{Hom}_{D_{A}}\left(W^{(\Lambda)}(A), E\right)$ is isomorphic to $\operatorname{Ker}\left[\mathbb{F}-\left[\Lambda^{p-1}\right]: E \rightarrow E\right]$;

(2) $\operatorname{Ext}_{D_{A}}^{1}\left(W^{(\Lambda)}(A), E\right)$ is isomorphic to Coker $\left[\mathbb{F}-\left[\Lambda^{p-1}\right]: E \rightarrow E\right]$;

(3) $\operatorname{Ext}_{D_{A}}^{j}\left(W^{(\Lambda)}(A), E\right)=0$ for $j>1$.

EXAmple 4.7 . Let $A$ be a $\mathbb{Z}[\Lambda, M]$-algebra. Then $\varphi \mapsto \varphi\left([1]_{\Lambda}\right)$ gives rise to an isomorphism

$$
\operatorname{Hom}_{D_{A}}\left(W^{(\Lambda)}(A), W^{(M)}(A)\right) \stackrel{\sim}{\longrightarrow} \operatorname{Ker}\left[F^{(M)}-\left[\Lambda^{p-1}\right]: W^{(M)}(A) \rightarrow W^{(M)}(A)\right] .
$$

Putting $\boldsymbol{a}=\varphi([1])$, we obtain

$$
\varphi(\boldsymbol{u})=\varphi\left(\sum_{k=0}^{\infty} V^{k}\left[u_{k}\right]\right)=\sum_{k=0}^{\infty} V^{k} \varphi\left(\left[u_{k}\right]\right)=\sum_{k=0}^{\infty} V^{k}\left(\left[u_{k}\right] \cdot \boldsymbol{a}\right)
$$

for $\boldsymbol{u}=\left(u_{0}, u_{1}, u_{2}, \ldots\right) \in W^{(\Lambda)}(A)$.

REMARK 4.8. Let $A$ be a $\mathbb{Z}[\Lambda, M]$-algebra, and let $\boldsymbol{a}=\left(a_{0}, a_{1}, a_{2}, \ldots\right) \in$ $W^{(\Lambda)}(A), \boldsymbol{b}=\left(b_{0}, b_{1}, b_{2}, \ldots\right) \in W^{(M)}(A)$. We define $\langle\boldsymbol{a}, \boldsymbol{b}\rangle_{\Lambda, M} \in W^{(M)}(A)$ by

$$
\langle\boldsymbol{a}, \boldsymbol{b}\rangle_{\Lambda, M}=\sum_{k=0}^{\infty} V^{k}\left(\left[a_{k}\right] \boldsymbol{b}\right)=\sum_{k=0}^{\infty} V^{k}\left(\sum_{i=0}^{k}\left[a_{i}^{p^{k-i}} b_{k-i}\right]\right),
$$

adopting the formula at the end of 4.7. Putting

$$
\boldsymbol{A}=\sum_{k=0}^{\infty} \mathbb{V}^{k}\left[a_{k}\right], \quad \boldsymbol{B}=\sum_{k=0}^{\infty} \mathbb{V}^{k}\left[b_{k}\right]
$$

we have

$$
\boldsymbol{A}[1]_{\Lambda}=\boldsymbol{a}, \quad \boldsymbol{B}[1]_{M}=\boldsymbol{b}
$$


and

$$
(\boldsymbol{A B})[1]_{M}=\langle\boldsymbol{a}, \boldsymbol{b}\rangle_{\Lambda, M}
$$

It is readily seen that:

(1) $\left\langle\boldsymbol{a}, \boldsymbol{b}+\boldsymbol{b}^{\prime}\right\rangle_{\Lambda, M}=\langle\boldsymbol{a}, \boldsymbol{b}\rangle_{\Lambda, M}+\left\langle\boldsymbol{a}, \boldsymbol{b}^{\prime}\right\rangle_{\Lambda, M}$;

(2) $\left\langle\boldsymbol{a}+\boldsymbol{a}^{\prime}, \boldsymbol{b}\right\rangle_{\Lambda, M}=\langle\boldsymbol{a}, \boldsymbol{b}\rangle_{\Lambda, M}+\left\langle\boldsymbol{a}^{\prime}, \boldsymbol{b}\right\rangle_{\Lambda, M}$ if $F^{(M)} \boldsymbol{b}=\left[\Lambda^{p-1}\right] \boldsymbol{b}$.

ExAmple 4.9 . Let $A$ be a $\mathbb{Z}[\Lambda, M]$-algebra. Then the isomorphism

$$
\operatorname{Ext}_{D_{A}}^{1}\left(W^{(\Lambda)}(A), W^{(M)}(A)\right) \stackrel{\sim}{\longrightarrow} \operatorname{Coker}\left[F^{(M)}-\left[\Lambda^{p-1}\right]: W^{(M)}(A) \rightarrow W^{(M)}(A)\right]
$$

is described explicitly as follows.

Let $C \in D_{A}$, and define a left $D_{A}$-endomorphism $U_{\Lambda, M, C}$ of $D_{A}^{2}$ by

$$
U_{\Lambda, M, \boldsymbol{C}}:(\boldsymbol{P}, \boldsymbol{Q}) \mapsto(\boldsymbol{P}, \boldsymbol{Q})\left(\begin{array}{cc}
\mathbb{F}-\left[\Lambda^{p-1}\right] & -\boldsymbol{C} \\
0 & \mathbb{F}-\left[M^{p-1}\right]
\end{array}\right)
$$

Then we have gotten a commutative diagram of left $D_{A}$-modules with exact rows

$$
\begin{aligned}
& 0 \longrightarrow D_{A} \stackrel{i}{\longrightarrow} D_{A} \oplus D_{A} \stackrel{j}{\longrightarrow} D_{A} \longrightarrow 0 \\
& \downarrow \cdot\left(\mathbb{F}-\left[M^{p-1}\right]\right) \quad \downarrow U_{\Lambda, M, C} \quad \downarrow \cdot\left(\mathbb{F}-\left[\Lambda^{p-1}\right]\right) \\
& 0 \longrightarrow D_{A} \longrightarrow D_{A} \oplus D_{A} \longrightarrow D_{A} \longrightarrow 0 \text {. }
\end{aligned}
$$

Here $i$ and $j$ are defined by $i: \boldsymbol{Q} \mapsto(0, \boldsymbol{Q})$ and $j:(\boldsymbol{P}, \boldsymbol{Q}) \mapsto \boldsymbol{P}$. Taking the cokernels of the vertical arrows, we obtain an extension of $D_{A}$-modules

$$
0 \rightarrow W^{(M)}(A) \rightarrow E \rightarrow W^{(\Lambda)}(A) \rightarrow 0
$$

Proposition 4.9.1. Put $\boldsymbol{c}=\boldsymbol{C}[1]_{M} \in W^{(M)}(A)$. The class of $E$ in $\operatorname{Ext}_{D_{A}}^{1}\left(W^{(\Lambda)}(A), W^{(M)}(A)\right)$ coincides with the class of $\boldsymbol{c}$ in $\operatorname{Coker}\left[F^{(M)}-\left[\Lambda^{p-1}\right]\right.$ : $\left.W^{(M)}(A) \rightarrow W^{(M)}(A)\right]$.

Proof. Let $\varphi_{(M, c)}: D_{A} \rightarrow W^{(M)}(A)$ be a $D_{A}$-homomorphism defined by $\boldsymbol{P} \mapsto \boldsymbol{P} \boldsymbol{c}$. Then an extension representing

$$
[c] \in \operatorname{Coker}\left[F^{(M)}-\left[\Lambda^{p-1}\right]: W^{(M)}(A) \rightarrow W^{(M)}(A)\right]=H_{0}^{2}\left(\widehat{\mathcal{G}}_{A}^{(\Lambda)}, \widehat{\mathcal{G}}_{A}^{(M)}\right)
$$

is given by the push-down

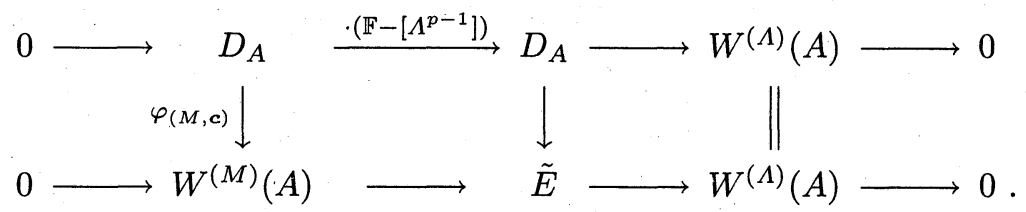


Define now $i^{\prime}: D_{A} \rightarrow D_{A} \oplus D_{A}$ by

$$
\boldsymbol{Q} \mapsto\left(0, \boldsymbol{Q}\left(\mathbb{F}-\left[M^{p-1}\right]\right)\right)
$$

and $j^{\prime}: D_{A} \oplus D_{A} \rightarrow D_{A} \oplus W^{(M)}(A)$ by

$$
(\boldsymbol{P}, \boldsymbol{Q}) \mapsto\left(\boldsymbol{P}, \varphi_{M}(\boldsymbol{Q})\right)=\left(\boldsymbol{P}, \boldsymbol{Q}[1]_{M}\right) .
$$

Then we have a commutative diagram with exact rows

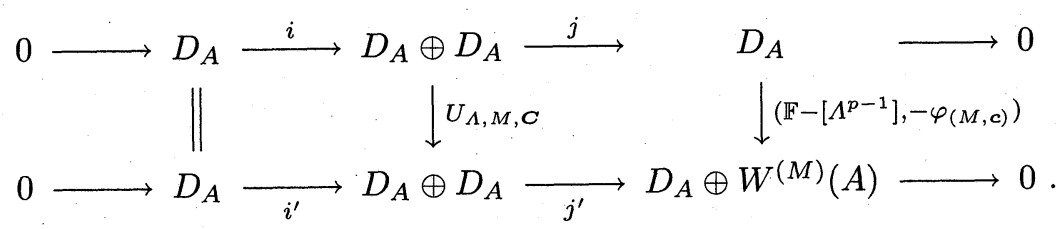

Taking the cokernels of the vertical arrows, we obtain the desired isomorphism $E \stackrel{\sim}{\rightarrow} \tilde{E}$.

REMARK 4.10. Let $\tilde{E}$ be the extension of $W^{(\Lambda)}(A)$ by $W^{(M)}(A)$ defined above. We can describe $\tilde{E}$ in terms of 2-cocycles as follows.

At first consider the extension of $D_{A}$-module

$$
0 \rightarrow D_{A} \stackrel{\cdot\left(\mathbb{F}-\left[\Lambda^{p-1}\right]\right)}{\longrightarrow} D_{A} \stackrel{\varphi_{\Lambda}}{\longrightarrow} W^{(\Lambda)}(A) \rightarrow 0 .
$$

Define a section $s: W^{(\Lambda)}(A) \rightarrow D_{A}$ of $\varphi_{\Lambda}$ by

$$
s(\boldsymbol{a})=\sum_{k=0}^{\infty} \mathbb{V}^{k}\left[a_{k}\right]
$$

where $\boldsymbol{a}=\left(a_{0}, a_{1}, a_{2}, \ldots\right)$. Then the 2-cocycle $\boldsymbol{Q} \in Z^{2}\left(W^{(\Lambda)}(A), D_{A}\right)$ associated to the section $s$ is given by

$$
\boldsymbol{Q}\left(\boldsymbol{a}, \boldsymbol{a}^{\prime}\right)\left(\mathbb{F}-\left[\Lambda^{p-1}\right]\right)=s(\boldsymbol{a})+s\left(\boldsymbol{a}^{\prime}\right)-s\left(\boldsymbol{a}+\boldsymbol{a}^{\prime}\right) .
$$

Note now that $\tilde{\boldsymbol{c}}=\varphi_{(M, c)} \circ \boldsymbol{Q} \in Z^{2}\left(W^{(\Lambda)}(A), W^{(M)}(A)\right)$ is defined by

$$
\tilde{c}\left(\boldsymbol{a}, \boldsymbol{a}^{\prime}\right)=\boldsymbol{Q}\left(\boldsymbol{a}, \boldsymbol{a}^{\prime}\right) \boldsymbol{c}
$$

Hence the extension $\tilde{E}$ of $W^{(\Lambda)}(A)$ by $W^{(M)}(A)$ is written as $\tilde{E}=W^{(\Lambda)}(A) \times$ $W^{(M)}(A)$ with the addition

$$
(\boldsymbol{a}, \boldsymbol{b})+\left(\boldsymbol{a}^{\prime}, \boldsymbol{b}^{\prime}\right)=\left(\boldsymbol{a}+{ }^{(\Lambda)} \boldsymbol{a}^{\prime}, \boldsymbol{b}+{ }^{(M)} \boldsymbol{b}^{\prime}+{ }^{(M)} \tilde{\boldsymbol{c}}\left(\boldsymbol{a}, \boldsymbol{a}^{\prime}\right)\right) .
$$


It is easily verified that, if $\boldsymbol{c}=\left(F^{(M)}-\left[\Lambda^{p-1}\right]\right) \boldsymbol{b}$,

$$
\tilde{\boldsymbol{c}}\left(\boldsymbol{a}, \boldsymbol{a}^{\prime}\right)=\langle\boldsymbol{a}, \boldsymbol{b}\rangle_{\Lambda, M}+\left\langle\boldsymbol{a}^{\prime}, \boldsymbol{b}\right\rangle_{\Lambda, M}-\left\langle\boldsymbol{a}+\boldsymbol{a}^{\prime}, \boldsymbol{b}\right\rangle_{\Lambda, M}
$$

EXAMPLE 4.11. Let

$$
A=\mathbb{Z}\left[\Lambda, M, U_{0}, U_{1}, U_{2}, \ldots, U_{0}^{\prime}, U_{1}^{\prime}, U_{2}^{\prime}, \ldots, V_{0}, V_{1}, V_{2}, \ldots\right]
$$

and

$\boldsymbol{U}=\left(U_{0}, U_{1}, U_{2}, \ldots\right), \boldsymbol{U}^{\prime}=\left(U_{0}^{\prime}, U_{1}^{\prime}, U_{2}^{\prime}, \ldots\right) \in W^{(\Lambda)}, \boldsymbol{V}=\left(V_{0}, V_{1}, V_{2}, \ldots\right) \in W^{(M)}$

We can describe explicitly $\tilde{\boldsymbol{V}}\left(\boldsymbol{U}, \boldsymbol{U}^{\prime}\right) \in W^{(M)}(A)$ as follows.

Put

$$
\boldsymbol{U}^{\prime \prime}=\left(U_{0}^{\prime \prime}, U_{1}^{\prime \prime}, U_{2}^{\prime \prime}, \ldots\right)=\boldsymbol{U}+{ }^{(\Lambda)} \boldsymbol{U}^{\prime} \in W^{(\Lambda)}(A)
$$

and

$$
\boldsymbol{Q}=\boldsymbol{Q}\left(\boldsymbol{U}, \boldsymbol{U}^{\prime}\right)=\sum_{k=1}^{\infty} \mathbb{V}^{k} \boldsymbol{q}_{k}, \quad \boldsymbol{q}_{k} \in W(A)
$$

Then we have

$$
V \boldsymbol{q}_{1}=\left[U_{0}\right]+\left[U_{0}^{\prime}\right]-\left[U_{0}^{\prime \prime}\right]
$$

and

$$
V \boldsymbol{q}_{k}=\left[U_{k-1}\right]+\left[U_{k-1}^{\prime}\right]-\left[U_{k-1}^{\prime \prime}\right]+\left[\Lambda^{p-1}\right] \boldsymbol{q}_{k-1}
$$

for $k \geq 2$ since

$$
\left(\sum_{k=1}^{\infty} \mathbb{V}^{k} \boldsymbol{q}_{k}\right)\left(\mathbb{F}-\left[\Lambda^{p-1}\right]\right)=\sum_{k=0}^{\infty} \mathbb{V}^{k}\left(\left[U_{k}\right]+\left[U_{k}^{\prime}\right]-\left[U_{k}^{\prime \prime}\right]\right)
$$

and

$$
\left(\sum_{k=1}^{\infty} \mathbb{V}^{k} \boldsymbol{q}_{k}\right)\left(\mathbb{F}-\left[\Lambda^{p-1}\right]\right)=V \boldsymbol{q}_{1}+\sum_{k=1}^{\infty} \mathbb{V}^{k}\left(V \boldsymbol{q}_{k+1}-\left[\Lambda^{p-1}\right] \boldsymbol{q}_{k}\right)
$$

(cf. the proof of Theorem 4.4). This implies that

$$
\Phi_{r}\left(\boldsymbol{q}_{1}\right)=\frac{1}{p}\left\{\Phi_{r+1}\left(\left[U_{0}\right]\right)+\Phi_{r+1}\left(\left[U_{0}^{\prime}\right]\right)-\Phi_{r+1}\left(\left[U_{0}^{\prime \prime}\right]\right)\right\}
$$

and

$$
\Phi_{r}\left(\boldsymbol{q}_{k}\right)=\frac{1}{p}\left\{\Phi_{r+1}\left(\left[U_{k-1}\right]\right)+\Phi_{r+1}\left(\left[U_{k-1}^{\prime}\right]\right)-\Phi_{r+1}\left(\left[U_{k-1}^{\prime \prime}\right]\right)+\Phi_{r+1}\left(\left[\Lambda^{p-1}\right] \boldsymbol{q}_{k-1}\right)\right\}
$$


Noting that

$$
\begin{aligned}
\Phi_{r+k}^{(\Lambda)}\left(U_{0}, \ldots, U_{k-2}, 0, \ldots, 0\right)+p^{k-1} \Phi_{r+1}^{(\Lambda)}\left(\left[U_{k-1}\right]\right) \\
=\Phi_{r+k}^{(\Lambda)}\left(U_{0}, \ldots, U_{k-2}, U_{k-1}, 0, \ldots, 0\right)
\end{aligned}
$$

we obtain

$$
\begin{aligned}
\Phi_{r}\left(\boldsymbol{q}_{k}\right) \\
=\frac{1}{p^{k} \Lambda^{p^{r+1}-1}}\left\{\Phi_{r+k}^{(\Lambda)}\left(U_{0}, U_{1}, \ldots, U_{k-1}, 0, \ldots, 0\right)\right. \\
\left.\quad+\Phi_{r+k}^{(\Lambda)}\left(U_{0}^{\prime}, U_{1}^{\prime}, \ldots, U_{k-1}^{\prime}, 0, \ldots, 0\right)-\Phi_{r+k}^{(\Lambda)}\left(U_{0}^{\prime \prime}, U_{1}^{\prime \prime}, \ldots, U_{k-1}^{\prime \prime}, 0, \ldots, 0\right)\right\} \\
=\frac{1}{p^{k} \Lambda^{p^{r+1}}}\left\{\Phi_{r+k}\left(\Lambda U_{0}, \Lambda U_{1}, \ldots, \Lambda U_{k-1}, 0, \ldots, 0\right)\right. \\
\left.\quad+\Phi_{r+k}\left(\Lambda U_{0}^{\prime}, \Lambda U_{1}^{\prime}, \ldots, \Lambda U_{k-1}^{\prime}, 0, \ldots, 0\right)-\Phi_{r+k}\left(\Lambda U_{0}^{\prime \prime}, \Lambda U_{1}^{\prime \prime}, \ldots, \Lambda U_{k-1}^{\prime \prime}, 0, \ldots, 0\right)\right\}
\end{aligned}
$$

for $k \geq 1$ and $r \geq 0$. Put now

$$
\boldsymbol{Z}=\tilde{\boldsymbol{V}}\left(\boldsymbol{U}, \boldsymbol{U}^{\prime}\right)=\sum_{k=1}^{\infty} V^{k}\left(\boldsymbol{q}_{k} \cdot \boldsymbol{V}\right) \in W^{(M)}(A)
$$

Then we have

$$
\begin{aligned}
& \Phi_{r}^{(M)}(\boldsymbol{Z})=\sum_{k=1}^{r} p^{k} \Phi_{r-k}\left(\boldsymbol{q}_{k}\right) \Phi_{r-k}^{(M)}(\boldsymbol{V}) \\
&=\sum_{k=1}^{r} \frac{1}{\Lambda^{p^{r-k+1}}}\left\{\Phi_{r}\left(\Lambda U_{0}, \Lambda U_{1}, \ldots, \Lambda U_{k-1}, 0, \ldots, 0\right)\right. \\
&+ \Phi_{r}\left(\Lambda U_{0}^{\prime}, \Lambda U_{1}^{\prime}, \ldots, \Lambda U_{k-1}^{\prime}, 0, \ldots, 0\right) \\
&\left.-\Phi_{r}\left(\Lambda U_{0}^{\prime \prime}, \Lambda U_{1}^{\prime \prime}, \ldots, \Lambda U_{k-1}^{\prime \prime}, 0, \ldots, 0\right)\right\} \Phi_{r-k}^{(M)}(\boldsymbol{V}) .
\end{aligned}
$$

Remark 4.12 . Let $A$ be a $\mathbb{Z}[M]$-algebra. Then the $D_{A}$-homomorphism $\alpha^{(M)}: W^{(M)}(A) \rightarrow W(A)$ is represented by a morphism of complexes

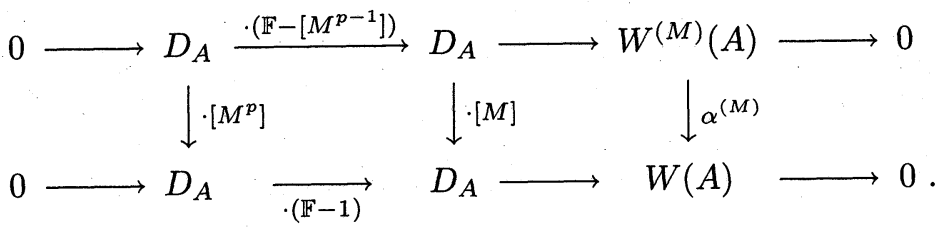

From now, we give an interpretation of $W^{(M)}(A)$ as the Cartier module of the formal group $\widehat{\mathcal{G}}_{A}^{(M)}$. 
4.13. Let $A$ be a $\mathbb{Z}_{(p)}$-algebra, and let $G$ be a commutative formal group over $A$. Then it is well known that $\operatorname{Hom}_{A-\mathrm{gr}}\left(\widehat{W}_{A}, G\right)$ is isomorphic to the Cartier module $C(G)$ of $p$-typical curves of $G$ (the first theorem of the Cartier theory on $p$-typical curves, cf. [4, Th. 27.7.5]).

$C(G)=\operatorname{Hom}_{A-\mathrm{gr}}\left(\widehat{W}_{A}, G\right)$ has a structure of right End ${ }_{A-\mathrm{gr}} \widehat{W}_{A}$-module by the composition of morphisms. Therefore $C(G)$ has a structure of left $D_{A}$-module since End $_{A-\mathrm{gr}} \widehat{W}_{A}$ is anti-isomorphic to $D_{A}$, as is remarked in 4.2.1.

ExAMPLE 4.14. Let $\boldsymbol{U}=\left(U_{0}, U_{1}, U_{2}, \ldots\right)$ and $\boldsymbol{T}=\left(T_{0}, T_{1}, T_{2}, \ldots\right)$. Define a formal power series $E_{p}(\boldsymbol{U} ; \boldsymbol{T}) \in \mathbb{Z}_{(p)}\left[U_{0}, U_{1}, U_{2}, \ldots\right]\left[\left[T_{0}, T_{1}, T_{2}, \ldots\right]\right]$ by

$$
E_{p}(\boldsymbol{U} ; \boldsymbol{T})=\exp \left(\sum_{r=0}^{\infty} \frac{\Phi_{r}(\boldsymbol{U}) \Phi_{r}(\boldsymbol{T})}{p^{r}}\right)=\exp \left(\sum_{r=0}^{\infty} \frac{\Phi_{r}(P(\boldsymbol{U}, \boldsymbol{T}))}{p^{r}}\right)
$$

It is verified without difficulty that

(1) $E_{p}(S(\boldsymbol{U}, \boldsymbol{V}) ; \boldsymbol{T})=E_{p}(\boldsymbol{U} ; \boldsymbol{T}) E_{p}(\boldsymbol{V} ; \boldsymbol{T})$;

(2) $E_{p}(\boldsymbol{U} ; S(\boldsymbol{V}, \boldsymbol{T}))=E_{p}(\boldsymbol{U} ; \boldsymbol{V}) E_{p}(\boldsymbol{U} ; \boldsymbol{T})$;

(3) $E_{p}(P(\boldsymbol{U}, \boldsymbol{V}) ; \boldsymbol{T})=E_{p}(\boldsymbol{V} ; P(\boldsymbol{U}, \boldsymbol{T}))$;

(4) $E_{p}(F(\boldsymbol{U}) ; \boldsymbol{T})=E_{p}(\boldsymbol{U} ; V(\boldsymbol{T}))$;

(5) $E_{p}(V(\boldsymbol{U}) ; \boldsymbol{T})=E_{p}(\boldsymbol{U} ; F(\boldsymbol{T}))$.

Let $A$ be a $\mathbb{Z}_{(p)}$-algebra. Then it is known that $\boldsymbol{a} \mapsto E_{p}(\boldsymbol{a} ; \boldsymbol{T})$ gives rise to a $D_{A}$-isomorphism $\eta: W(A) \stackrel{\sim}{\rightarrow} C\left(\widehat{\mathbb{G}}_{m, A}\right)=\operatorname{Hom}_{A-\mathrm{gr}}\left(\widehat{W}_{A}, \widehat{\mathbb{G}}_{m, A}\right)$ (cf. $\left.[4,17.2 .12]\right)$.

4.15. Let $A$ be a $\mathbb{Z}[\Lambda, M]$-algebra and $B=A[t] /\left(t^{2}-M t\right)$. Let $\varepsilon$ denote the image of $t$ in $B$. Then we have $\varepsilon^{2}=M \varepsilon$. The exact sequence of formal groups

$$
0 \rightarrow \widehat{\mathcal{G}}_{A}^{(M)} \rightarrow\left(\widehat{\prod_{B / A} \mathbb{G}_{m, B}}\right) \rightarrow \widehat{\mathbb{G}}_{m, A} \rightarrow 0
$$

induces an exact sequence of Cartier modules

$$
0 \rightarrow C\left(\widehat{\mathcal{G}}_{A}^{(M)}\right) \rightarrow C\left(\widehat{\mathbb{G}}_{m, B}\right) \rightarrow C\left(\widehat{\mathbb{G}}_{m, A}\right) \rightarrow 0
$$

More precisely,

$$
C\left(\widehat{\mathbb{G}}_{m, B}\right)=\operatorname{Hom}_{A-\mathrm{gr}}\left(\widehat{W}_{B}, \widehat{\mathbb{G}}_{m, B}\right) \rightarrow C\left(\widehat{\mathbb{G}}_{m, A}\right)=\operatorname{Hom}_{A-\mathrm{gr}}\left(\widehat{W}_{A}, \widehat{\mathbb{G}}_{m, A}\right)
$$

is induced from the ring homomorphism $B \rightarrow A$, and

$$
C\left(\widehat{\mathcal{G}}^{(M)}\right)=\operatorname{Hom}_{A-\mathrm{gr}}\left(\widehat{W}_{A}, \widehat{\mathcal{G}}^{(M)}\right) \rightarrow C\left(\widehat{\mathbb{G}}_{m, B}\right)=\operatorname{Hom}_{B-\mathrm{gr}}\left(\widehat{W}_{B}, \widehat{\mathbb{G}}_{m, B}\right)
$$


is defined by

$$
F(\boldsymbol{T}) \mapsto 1+\varepsilon F(\boldsymbol{T})
$$

On the other hand, we have an exact sequence of left $D_{A}$-modules

$$
0 \rightarrow W^{(M)}(A) \rightarrow W(B) \rightarrow W(A) \rightarrow 0
$$

by Theorem 1.21 . Since the diagram

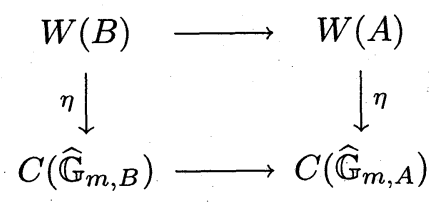

is commutative, we obtain a commutative diagram of left $D_{A}$-modules with exact rows

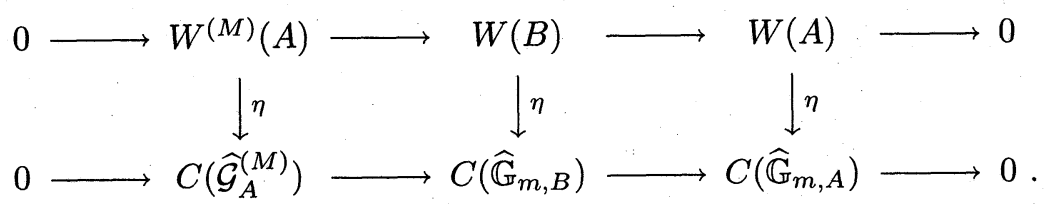

We have gotten the following theorem, noting $\eta: W(B) \rightarrow C\left(\widehat{\mathbb{G}}_{m, B}\right)$ and $\eta$ : $W(A) \rightarrow C\left(\widehat{\mathbb{G}}_{m, A}\right)$ are bijective.

Theorem 4.15.1. Let $A$ be a $\mathbb{Z}_{(p)}[M]$-algebra. Then there exists a $D_{A^{-}}$ isomorphism

$$
\eta: W^{(M)}(A) \stackrel{\sim}{\rightarrow} C\left(\widehat{\mathcal{G}}^{(M)}\right)=\operatorname{Hom}_{A-\mathrm{gr}}\left(\widehat{W}_{A}, \widehat{\mathcal{G}}^{(M)}\right)
$$

4.16. Now we introduce formal power series to describe explicitly the isomorphism $\eta: W^{(M)}(A) \stackrel{\sim}{\rightarrow} \operatorname{Hom}_{A-\mathrm{gr}}\left(\widehat{W}_{A}, \widehat{\mathcal{G}}^{(M)}\right)$.

Let $\boldsymbol{U}=\left(U_{0}, U_{1}, U_{2}, \ldots\right)$ and $\boldsymbol{T}=\left(T_{0}, T_{1}, T_{2}, \ldots\right)$. We define a formal power series

$$
E_{p}^{(M)}(\boldsymbol{U} ; \boldsymbol{T}) \in \mathbb{Z}_{(p)}[M]\left[U_{0}, U_{1}, U_{2}, \ldots\right]\left[\left[T_{0}, T_{1}, T_{2}, \ldots\right]\right]
$$

by

$$
E_{p}^{(M)}(\boldsymbol{U} ; \boldsymbol{T})=\frac{1}{M}\left[E_{p}\left(\alpha^{(M)} \boldsymbol{U} ; \boldsymbol{T}\right)-1\right]
$$

EXAMPLE 4.17.1. $E_{p}^{(1)}(\boldsymbol{U} ; \boldsymbol{T})=E_{p}(\boldsymbol{U} ; \boldsymbol{T})-1$.

EXAMPLE 4.17.2. $E_{p}^{(0)}(\boldsymbol{U} ; \boldsymbol{T})=\sum_{r=0}^{\infty} U_{r} \Phi_{r}(\boldsymbol{T})$.

Indeed, by the definition, we have

$$
\Phi_{r}\left(M U_{0}, \ldots, M U_{r}\right) \equiv p^{r} M U_{r} \bmod M^{p}
$$


It follows that

$$
\begin{aligned}
\log E_{p}\left(\alpha^{(M)} \boldsymbol{U}: \boldsymbol{T}\right) & =\sum_{r=0}^{\infty} \frac{\Phi_{r}\left(M U_{0}, \ldots, M U_{r}\right) \Phi_{r}(\boldsymbol{T})}{p^{r}} \\
& \equiv 1+M\left(\sum_{r=0}^{\infty} U_{r} \Phi_{r}(\boldsymbol{T})\right) \bmod M^{p}
\end{aligned}
$$

Hence we obtain

$$
E_{p}\left(\alpha^{(M)} \boldsymbol{U}: \boldsymbol{T}\right) \equiv 1+M\left(\sum_{r=0}^{\infty} U_{r} \Phi_{r}(\boldsymbol{T})\right) \bmod M^{2}
$$

and

$$
E_{p}^{(M)}(\boldsymbol{U} ; \boldsymbol{T})=\frac{1}{M}\left[E_{p}\left(\alpha^{(M)} \boldsymbol{U} ; \boldsymbol{T}\right)-1\right] \equiv \sum_{r=0}^{\infty} U_{r} \Phi_{r}(\boldsymbol{T}) \bmod M
$$

Proposition 4.18. Let $\boldsymbol{U}=\left(U_{0}, U_{1}, U_{2}, \ldots\right), \boldsymbol{V}=\left(V_{0}, V_{1}, V_{2}, \ldots\right)$ and $\boldsymbol{T}=$ $\left(T_{0}, T_{1}, T_{2}, \ldots\right)$. Then:

(1) $E_{p}^{(M)}\left(\boldsymbol{U}+{ }^{(M)} \boldsymbol{V} ; \boldsymbol{T}\right)=E_{p}^{(M)}(\boldsymbol{U} ; \boldsymbol{T})+E_{p}^{(M)}(\boldsymbol{V} ; \boldsymbol{T})+M E_{p}^{(M)}(\boldsymbol{U} ; \boldsymbol{T}) E_{p}^{(M)}(\boldsymbol{V} ; \boldsymbol{T})$;

(2) $E_{p}^{(M)}(\boldsymbol{U} ; \boldsymbol{V}+\boldsymbol{T})=E_{p}^{(M)}(\boldsymbol{U} ; \boldsymbol{V})+E_{p}^{(M)}(\boldsymbol{U} ; \boldsymbol{T})+M E_{p}^{(M)}(\boldsymbol{U} ; \boldsymbol{V}) E_{p}^{(M)}(\boldsymbol{U} ; \boldsymbol{T})$;

(3) $E_{p}^{(M)}\left(\boldsymbol{V} \cdot{ }^{(M)} \boldsymbol{U} ; \boldsymbol{T}\right)=E_{p}^{(M)}(\boldsymbol{U} ; \boldsymbol{V T})$;

(4) $E_{p}^{(M)}\left(F^{(M)}(\boldsymbol{U}) ; \boldsymbol{T}\right)=E_{p}^{(M)}(\boldsymbol{U} ; V(\boldsymbol{T}))$;

(5) $E_{p}^{(M)}(V(\boldsymbol{U}) ; \boldsymbol{T})=E_{p}^{(M)}(\boldsymbol{U} ; F(\boldsymbol{T}))$.

Proof. The assertion (2) follows immediately from 4.14(2). We can deduce (1), (3), (4) and (5) from 4.14(1), 4.14(3), 4.14(4) and 4.14(5), combining the formulas

$$
\begin{aligned}
\alpha^{(M)}\left(\boldsymbol{U}+{ }^{(M)} \boldsymbol{V}\right) & =\alpha^{(M)}(\boldsymbol{U})+\alpha^{(M)}(\boldsymbol{V}), \\
\alpha^{(M)}\left(\boldsymbol{V}{ }^{(M)} \boldsymbol{U}\right) & =\boldsymbol{V} \cdot \alpha^{(M)}(\boldsymbol{U}), \\
\alpha^{(M)}\left(F^{(M)}(\boldsymbol{U})\right) & =F\left(\alpha^{(M)}((\boldsymbol{U})),\right. \\
\alpha^{(M)}(V(\boldsymbol{U})) & =V\left(\alpha^{(M)}((\boldsymbol{U})),\right.
\end{aligned}
$$

respectively.

Proposition 4.19. Let $A$ be a $\mathbb{Z}_{(p)}[M]$-algebra. Then the isomorphism $\eta$ : $W^{(M)}(A) \stackrel{\sim}{\rightarrow} C\left(\widehat{\mathcal{G}}^{(M)}\right)=\operatorname{Hom}_{A-\mathrm{gr}}\left(\widehat{W}_{A}, \widehat{\mathcal{G}}^{(M)}\right)$ is given by $\boldsymbol{a} \mapsto E_{p}^{(M)}(\boldsymbol{a} ; \boldsymbol{T})$.

Proof. This is a direct consequence of the following lemma. 
Lemma 4.20. Let $A$ be $a \mathbb{Z}_{(p)}[M]$-algebra and $B=A[t] /\left(t^{2}-M t\right)$, and let $\varepsilon$ denote the image of $t$ in $B$. Let $\boldsymbol{a} \in W^{(M)}$, and put $\varepsilon \boldsymbol{a}=\left(\varepsilon a_{0}, \varepsilon a_{1}, \varepsilon a_{2}, \ldots\right)$. Then:

$$
1+\varepsilon E_{p}^{(M)}(\boldsymbol{a} ; \boldsymbol{T})=E_{p}(\varepsilon \boldsymbol{a} ; \boldsymbol{T}) .
$$

Proof. We can prove the assertion similarly as in the proof of Lemma 3.7, and thus omit the details.

EXAmple 4.21 . Let $A$ be a $\mathbb{Z}_{(p)}[M]$-algebra. Then the additive group $C\left(\widehat{\mathbb{G}}_{a, A}\right)=W^{(0)}(A)$ is isomorphic to the direct product $A^{\mathbb{N}}$, and $\mathbb{F}$ and $\mathbb{V}$ on $C\left(\widehat{\mathbb{G}}_{a, A}\right)$ are given by

$$
F^{(0)}:\left(a_{0}, a_{1}, a_{2}, \ldots\right) \mapsto\left(p a_{1}, p a_{2}, p a_{3}, \ldots\right)
$$

and

$$
V:\left(a_{0}, a_{1}, a_{2}, \ldots\right) \mapsto\left(0, a_{0}, a_{1}, a_{2}, \ldots\right),
$$

respectively, as is remarked in 1.9.2. Moreover, we have

$$
E_{p}^{(0)}(\boldsymbol{a} ; \boldsymbol{T})=\sum_{r=0}^{\infty} a_{r} \Phi_{r}(\boldsymbol{T})
$$

for $\boldsymbol{a} \in W^{(0)}(A)=C\left(\widehat{\mathbb{G}}_{a, A}\right)($ Example 4.17.2).

In particular, $[1]_{0} \in W^{(0)}(A)$ corresponds to the restriction map $R: \widehat{W}_{A} \rightarrow$ $\widehat{W}_{1, A}=\widehat{\mathbb{G}}_{a, A}$ since $E_{p}^{(0)}([1] ; \boldsymbol{T})=T_{0}$.

Hereafter we investigate the isomorphisms presented in Theorem 3.5.1 via the Cartier theory.

Lemma 4.22 . Let $A$ be a $\mathbb{Z}[\Lambda]$-algebra, $\boldsymbol{a}=\left(a_{0}, a_{1}, a_{2}, \ldots\right) \in W(A)$ and $\boldsymbol{u}=\left(u_{0}, u_{1}, u_{2}, \ldots\right) \in W^{(\Lambda)}(A)$. If $F(\boldsymbol{a})=\left[\Lambda^{p-1}\right] \boldsymbol{a}$,

$$
E_{p}\left(\boldsymbol{a}, \Lambda ; E_{p}^{(\Lambda)}(\boldsymbol{u} ; \boldsymbol{T})\right)=E_{p}\left(\langle\boldsymbol{u}, \boldsymbol{a}\rangle_{\Lambda, 1} ; \boldsymbol{T}\right)
$$

Here

$$
\langle\boldsymbol{u}, \boldsymbol{a}\rangle_{\Lambda, 1}=\sum_{k=0}^{\infty} V^{k}\left(\left[u_{k}\right] \boldsymbol{a}\right) \in W(A)
$$

Proof. We may assume that $A$ is flat over $\mathbb{Z}[\Lambda]$ since $\operatorname{Ker}\left[F-\left[\Lambda^{p-1}\right]\right.$ : $\left.W_{\mathbb{Z}[\Lambda]} \rightarrow W_{\mathbb{Z}[\Lambda]}\right]$ is flat over $\mathbb{Z}[\Lambda]\left(\left[13\right.\right.$, Cor. 1.8]). Put now $a=\Phi_{0}(\boldsymbol{a})$. Then we have $\Phi_{r}(a)=\Lambda^{p^{r}-1} a$ for each $r>0$ and

$$
E_{p}(\boldsymbol{a}, \Lambda ; T)=(1+\Lambda T)^{a / \Lambda}
$$


Noting

$$
E_{p}^{(\Lambda)}(\boldsymbol{u} ; \boldsymbol{T})=\frac{1}{\Lambda}\left[\exp \left(\sum_{r=0}^{\infty} \frac{\Phi_{r}\left(\Lambda u_{0}, \Lambda u_{1}, \ldots, \Lambda u_{r}\right) \Phi_{r}(\boldsymbol{T})}{p^{r}}\right)-1\right]
$$

we see that

$$
\begin{aligned}
& E_{p}\left(\boldsymbol{a}, \Lambda ; E_{p}^{(\Lambda)}(\boldsymbol{u} ; \boldsymbol{T})\right) \\
& =\left[\exp \left(\sum_{r=0}^{\infty} \frac{\Phi_{r}\left(\Lambda u_{0}, \Lambda u_{1}, \ldots, \Lambda u_{r}\right) \Phi_{r}(\boldsymbol{T})}{p^{r}}\right)\right]^{a / \Lambda} \\
& =\exp \left(\sum_{r=0}^{\infty} \frac{a}{\Lambda} \frac{\Phi_{r}\left(\Lambda u_{0}, \Lambda u_{1}, \ldots, \Lambda u_{r}\right) \Phi_{r}(\boldsymbol{T})}{p^{r}}\right) \\
& =\exp \left(\sum_{r=0}^{\infty} \frac{\left(\Lambda^{p^{r}-1} u_{0}^{p^{r}}+p \Lambda^{p^{r-1}-1} u_{1}^{p^{r-1}}+\cdots+p^{r} u_{r}\right) a \Phi_{r}(\boldsymbol{T})}{p^{r}}\right) \\
& =\exp \left(\sum_{r=0}^{\infty} \frac{\left\{\Phi_{r}\left(\left[u_{0}\right]\right) \Phi_{r}(\boldsymbol{a})+p \Phi_{r-1}\left(\left[u_{1}\right]\right) \Phi_{r-1}(\boldsymbol{a})+\cdots+p^{r} \Phi_{0}\left(\left[u_{r}\right]\right) \Phi_{0}(\boldsymbol{a})\right\} \Phi_{r}(\boldsymbol{T})}{p^{r}}\right) \\
& =\exp \left(\sum_{r=0}^{\infty} \frac{\Phi_{r}\left(\left[u_{0}\right] \cdot \boldsymbol{a}+V\left(\left[u_{1}\right] \cdot \boldsymbol{a}\right)+\cdots+V^{r}\left(\left[u_{r}\right] \cdot \boldsymbol{a}\right)\right) \Phi_{r}(\boldsymbol{T})}{p^{r}}\right) \\
& =E_{p}\left(\sum_{k=0}^{\infty} V^{k}\left(\left[u_{k}\right] \cdot \boldsymbol{a}\right) ; \boldsymbol{T}\right) .
\end{aligned}
$$

Corollary 4.23. Let $A$ be a $\mathbb{Z}_{(p)}[\Lambda, M]$-algebra, $\boldsymbol{a} \in W^{(M)}(A)$ and $\boldsymbol{u} \in$ $W^{(\Lambda)}(A)$. If $F^{(M)}(\boldsymbol{a})=\left[\Lambda^{p-1}\right] \boldsymbol{a}$,

$$
E_{p}^{(M)}\left(\boldsymbol{a}, \Lambda ; E_{p}^{(\Lambda)}(\boldsymbol{u} ; \boldsymbol{T})\right)=E_{p}^{(M)}\left(\langle\boldsymbol{u}, \boldsymbol{a}\rangle_{\Lambda, M} ; \boldsymbol{T}\right)
$$

Here

$$
\langle\boldsymbol{u}, \boldsymbol{a}\rangle_{\Lambda, M}=\sum_{k=0}^{\infty} V^{k}\left(\left[u_{k}\right] \boldsymbol{a}\right) \in W^{(M)}(A)
$$

Proof. Let $B=A[t] /\left(t^{2}-M t\right)$, and let $\varepsilon$ denote the image of $t$ in $B$. We have $F(\varepsilon \boldsymbol{a})=\left[\Lambda^{p-1}\right](\varepsilon \boldsymbol{a})$ since $F^{(M)}(\boldsymbol{a})=\left[\Lambda^{p-1}\right] \boldsymbol{a}$ (cf. 1.19,(4)). Therefore, we obtain.

$$
E_{p}\left(\varepsilon \boldsymbol{a}, \Lambda ; E_{p}^{(\Lambda)}(\boldsymbol{u} ; \boldsymbol{T})\right)=E_{p}\left(\varepsilon\left(\sum_{k=0}^{\infty} V^{k}\left(\left[u_{k}\right] \cdot \boldsymbol{a}\right)\right) ; \boldsymbol{T}\right),
$$

combining 4.22 with 1.21 . Now note that

$$
E_{p}\left(\varepsilon \boldsymbol{a}, \Lambda ; E_{p}^{(\Lambda)}(\boldsymbol{u} ; \boldsymbol{T})\right)=1+\varepsilon E_{p}^{(M)}\left(\boldsymbol{a}, \Lambda ; E_{p}^{(\Lambda)}(\boldsymbol{u} ; \boldsymbol{T})\right)
$$


and

$$
E_{p}\left(\varepsilon\left(\sum_{k=0}^{\infty} V^{k}\left(\left[u_{k}\right] \cdot \boldsymbol{a}\right)\right) ; \boldsymbol{T}\right)=1+\varepsilon E_{p}^{(M)}\left(\sum_{k=0}^{\infty} V^{k}\left(\left[u_{k}\right] \cdot \boldsymbol{a}\right) ; \boldsymbol{T}\right)
$$

(cf. 3.7).

Now we can state the first main result on the compatibility of the isomorphism $\xi$ with the Cartier theory.

4.24. Let $A$ be a $\mathbb{Z}_{(p)}[\Lambda, M]$-algebra. By Theorem 3.5.1 we have an isomorphism

$$
\xi: \operatorname{Ker}\left[F^{(M)}-\left[\Lambda^{p-1}\right]: W^{(M)}(A) \rightarrow W^{(M)}(A)\right] \stackrel{\sim}{\longrightarrow} \operatorname{Hom}_{A-\operatorname{gr}}\left(\widehat{\mathcal{G}}_{A}^{(\Lambda)}, \widehat{\mathcal{G}}_{A}^{(M)}\right) .
$$

On the other hand, the Cartier theory asserts that $\gamma \mapsto C(\gamma)$ gives rise to an isomorphism

$$
\operatorname{Hom}_{A-\operatorname{gr}}\left(\widehat{\mathcal{G}}_{A}^{(\Lambda)}, \widehat{\mathcal{G}}_{A}^{(M)}\right) \stackrel{\sim}{\longrightarrow} \operatorname{Hom}_{D_{A}}\left(C\left(\widehat{\mathcal{G}}_{A}^{(\Lambda)}\right), C\left(\widehat{\mathcal{G}}_{A}^{(M)}\right)\right)
$$

(the second theorem of the Cartier theory on $p$-typical curves, cf. [4, Th. 27.7.10]). Now we have isomorphisms

$$
\eta: W^{(\Lambda)}(A) \stackrel{\sim}{\longrightarrow} C\left(\widehat{\mathcal{G}}_{A}^{(\Lambda)}\right)
$$

and

$$
\eta: W^{(M)}(A) \stackrel{\sim}{\longrightarrow} C\left(\widehat{\mathcal{G}}_{A}^{(M)}\right)
$$

by Theorem 4.15.1. Furthermore we have an isomorphism

$$
\operatorname{Hom}_{D_{A}}\left(W^{(\Lambda)}(A), W^{(M)}(A)\right) \stackrel{\sim}{\longrightarrow} \operatorname{Ker}\left[F^{(M)}-\left[\Lambda^{p-1}\right]: W^{(M)}(A) \rightarrow W^{(M)}(A)\right],
$$

defined by $\varphi \mapsto \varphi\left([1]_{\Lambda}\right)$, as is remarked in 4.7 .

Theorem 4.24.1. Let $\boldsymbol{a} \in \operatorname{Ker}\left[F^{(M)}-\left[\Lambda^{p-1}\right]: W^{(M)}(A) \rightarrow W^{(M)}(A)\right]$. Then we have

$$
C(\xi(a))=a
$$

under the identification

$$
\operatorname{Hom}_{D_{A}}\left(C\left(\widehat{\mathcal{G}}_{A}^{(\Lambda)}\right), C\left(\widehat{\mathcal{G}}_{A}^{(M)}\right)\right)=\operatorname{Ker}\left[F^{(M)}-\left[\Lambda^{p-1}\right]: W^{(M)}(A) \rightarrow W^{(M)}(A)\right]
$$

Proof. Let $\psi \in C\left(\widehat{\mathcal{G}}_{A}^{(\Lambda)}\right)=\operatorname{Hom}_{A-\mathrm{gr}}\left(\widehat{W}_{A}, \widehat{\mathcal{G}}_{A}^{(\Lambda)}\right)$ and $\gamma \in \operatorname{Hom}_{A-\operatorname{gr}}\left(\widehat{\mathcal{G}}_{A}^{(\Lambda)}, \widehat{\mathcal{G}}_{A}^{(M)}\right)$. Then $C(\gamma)(\psi)=\gamma \circ \psi$. Now recall that:

(1) $\eta(\boldsymbol{u}) \in C\left(\widehat{\mathcal{G}}_{A}^{(\Lambda)}\right)=\operatorname{Hom}_{A-\operatorname{gr}}\left(\widehat{W}_{A}, \widehat{\mathcal{G}}_{A}^{(\Lambda)}\right)$ is defined by $T \mapsto E_{p}^{(\Lambda)}(\boldsymbol{u} ; \boldsymbol{T})$, where $\boldsymbol{u} \in W^{(\Lambda)}(A)$ (Proposition 4.19); 
(2) $\xi(\boldsymbol{a}) \in \operatorname{Hom}_{A-\mathrm{gr}}\left(\widehat{\mathcal{G}}_{A}^{(\Lambda)}, \widehat{\mathcal{G}}_{A}^{(M)}\right)$ is defined by $T \mapsto E_{p}^{(M)}(\boldsymbol{a}, \Lambda ; T)$ (Remark $3.6)$.

Hence $C(\xi(\boldsymbol{a}))(\eta(\boldsymbol{u}))$ is defined by $T \mapsto E_{p}^{(M)}\left(\boldsymbol{a}, \Lambda ; E_{p}^{(\Lambda)}(\boldsymbol{u} ; \boldsymbol{T})\right)$. By Corollary 4.23, we have

$$
E_{p}^{(M)}\left(\boldsymbol{a}, \Lambda ; E_{p}^{(\Lambda)}(\boldsymbol{u} ; \boldsymbol{T})\right)=E_{p}^{(M)}\left(\langle\boldsymbol{u}, \boldsymbol{a}\rangle_{\Lambda, M} ; \boldsymbol{T}\right) .
$$

Then we get the conclusion, noting that $\left\langle[1]_{\Lambda}, \boldsymbol{a}\right\rangle_{\Lambda, M}=\boldsymbol{a}$.

REMARK 4.25. Let $A$ be a $\mathbb{Z}_{(p)}[\Lambda, M]$-algebra and $\boldsymbol{a} \in \operatorname{Ker}\left[F^{(M)}-\left[\Lambda^{p-1}\right]:\right.$ $\left.W^{(M)}(A) \rightarrow W^{(M)}(A)\right]$. Then the assertion of 4.23 is equivalent to the commutativity of the diagram

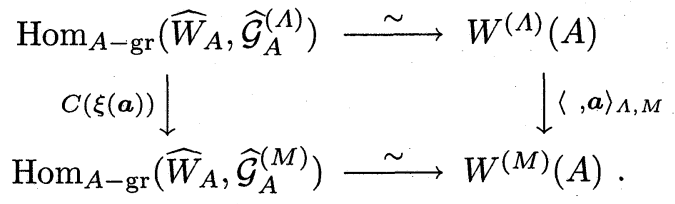

EXAMPLE 4.26 . Let $A$ be a $\mathbb{Z}_{(p)}[\Lambda]$-algebra. Then the diagram

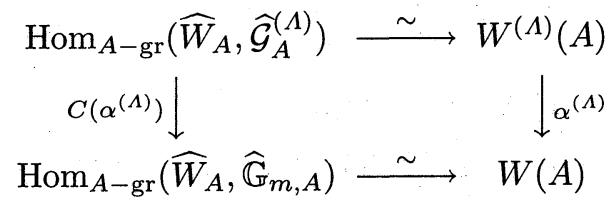

is commutative. Note that

$$
\alpha^{(\Lambda)}=\xi([\Lambda]): \widehat{\mathcal{G}}_{A}^{(\Lambda)} \rightarrow \widehat{\mathbb{G}}_{m, A}
$$

(cf. Example 3.8) and that

$$
\alpha^{(\Lambda)}=\langle,[\Lambda]\rangle_{\Lambda, 1}: W^{(\Lambda)}(A) \rightarrow W(A) .
$$

Corollary 4.27. Let $A$ be a $\mathbb{Z}_{(p)}[\Lambda, M, N]$-algebra and

$$
\begin{aligned}
& \boldsymbol{a} \in \operatorname{Ker}\left[F^{(M)}-\left[\Lambda^{p-1}\right]: W^{(M)}(A) \rightarrow W^{(M)}(A)\right], \\
& \boldsymbol{b} \in \operatorname{Ker}\left[F^{(N)}-\left[M^{p-1}\right]: W^{(N)}(A) \rightarrow W^{(N)}(A)\right] .
\end{aligned}
$$

Then the element of $\operatorname{Ker}\left[F^{(N)}-\left[\Lambda^{p-1}\right]: W^{(N)}(A) \rightarrow W^{(N)}(A)\right]$ corresponding to $\xi(\boldsymbol{b}) \circ \xi(\boldsymbol{a})$ is given by $\langle\boldsymbol{a}, \boldsymbol{b}\rangle_{M, N} \in W^{(N)}(A)$.

Proof. There exists uniquely $c \in \operatorname{Ker}\left[F^{(N)}-\left[\Lambda^{p-1}\right]: W^{(N)}(A) \rightarrow W^{(N)}(A)\right]$ such that $\xi(\boldsymbol{c})=\xi(\boldsymbol{b}) \circ \xi(\boldsymbol{a})$. Then we have

$$
C(\xi(\boldsymbol{c}))=C(\xi(\boldsymbol{b})) \circ C(\xi(\boldsymbol{a}))
$$


and, by the theorem,

$$
C(\xi(\boldsymbol{a}))=\langle, \boldsymbol{a}\rangle_{\Lambda, M}, C(\xi(\boldsymbol{b}))=\langle, \boldsymbol{b}\rangle_{M, N}, C(\xi(\boldsymbol{b}))=\langle, \boldsymbol{c}\rangle_{\Lambda, N} .
$$

Hence we obtain

$$
\langle, \boldsymbol{c}\rangle_{\Lambda, N}=\left\langle\langle, \boldsymbol{a}\rangle_{\Lambda, M}, \boldsymbol{b}\right\rangle_{M, N}
$$

and therefore

$$
\boldsymbol{c}=\left\langle[1]_{\Lambda}, \boldsymbol{c}\right\rangle_{\Lambda, N}=\left\langle\left\langle[1]_{\Lambda}, \boldsymbol{a}\right\rangle_{\Lambda, M}, \boldsymbol{b}\right\rangle_{M, N}=\langle\boldsymbol{a}, \boldsymbol{b}\rangle_{M, N}
$$

We conclude the article by giving a description of the Cartier module of an extension of $\widehat{\mathcal{G}}_{A}^{(\Lambda)}$ by $\widehat{\mathcal{G}}_{A}^{(M)}$.

4.28. Let $A$ be a $\mathbb{Z}_{(p)}[\Lambda, M]$-algebra and $c \in W^{(M)}(A)$. Let $\mathcal{E}$ denote the extension of $\widehat{\mathcal{G}}_{A}^{(\Lambda)}$ by $\widehat{\mathcal{G}}_{A}^{(M)}$ by the 2-cocycle

$$
C(X, Y)=F_{p}^{(M)}(\boldsymbol{c}, \Lambda ; X, Y) \in Z^{2}\left(\widehat{\mathcal{G}}_{A}^{(\Lambda)}, \widehat{\mathcal{G}}_{A}^{(M)}\right) .
$$

Then the group law of $\mathcal{E}=\operatorname{Spf} A\left[\left[T_{0}, T_{1}\right]\right]$ is given by

$$
\begin{aligned}
& T_{0} \mapsto T_{0} \otimes 1+1 \otimes T_{0}+\Lambda T_{0} \otimes T_{0} \\
& \begin{aligned}
& T_{1} \mapsto T_{1} \otimes 1+1 \otimes T_{1}+C\left(T_{0} \otimes 1,1 \otimes T_{0}\right) \\
& \quad \quad M\left\{T_{1} \otimes T_{1}+\left(T_{1} \otimes 1+1 \otimes T_{1}\right) C\left(T_{0} \otimes 1,1 \otimes T_{0}\right)\right\} \\
& \quad+M^{2}\left(T_{1} \otimes T_{1}\right) C\left(T_{0} \otimes 1,1 \otimes T_{0}\right) .
\end{aligned}
\end{aligned}
$$

Hence a homomorphism of formal groups

$$
\widehat{W}_{A}=\operatorname{Spf} A\left[\left[T_{0}, T_{1}, T_{2}, \ldots\right]\right] \longrightarrow \mathcal{E}=\operatorname{Spf} A\left[\left[T_{0}, T_{1}\right]\right]
$$

is given by

$$
T_{0} \mapsto F_{0}(\boldsymbol{T}), T_{1} \mapsto F_{1}(\boldsymbol{T}): A\left[\left[T_{0}, T_{1}\right]\right] \rightarrow A\left[\left[T_{0}, T_{1}, T_{2}, \ldots\right]\right],
$$

where $F_{0}(\boldsymbol{T})=F_{0}\left(T_{0}, T_{1}, T_{2}, \ldots\right)$ and $F_{1}(\boldsymbol{T})=F_{1}\left(T_{0}, T_{1}, T_{2}, \ldots\right) \in A\left[\left[T_{0}, T_{1}, T_{2}, \ldots\right]\right]$ satisfy the functional equations

$$
\begin{aligned}
F_{0}(\boldsymbol{X}+\boldsymbol{Y})= & F_{0}(\boldsymbol{X})+F_{0}(\boldsymbol{Y})+\Lambda F_{0}(\boldsymbol{X}) F_{0}(\boldsymbol{Y}) \\
F_{1}(\boldsymbol{X}+\boldsymbol{Y})= & F_{1}(\boldsymbol{X})+F_{1}(\boldsymbol{Y})+C\left(F_{0}(\boldsymbol{X}), F_{0}(\boldsymbol{Y})\right) \\
& +M\left\{F_{1}(\boldsymbol{X}) F_{1}(\boldsymbol{Y})+\left(F_{1}(\boldsymbol{X})+F_{1}(\boldsymbol{Y})\right) C\left(F_{0}(\boldsymbol{X}), F_{0}(\boldsymbol{Y})\right)\right\} \\
& +M^{2} F_{1}(\boldsymbol{X}) F_{1}(\boldsymbol{Y}) C\left(F_{0}(\boldsymbol{X}), F_{0}(\boldsymbol{Y})\right)
\end{aligned}
$$


Remark 4.28.1. Let $A$ be a $\mathbb{Z}_{(p)}[\Lambda]$-algebra and $c \in W(A)$. Let $\mathcal{E}$ denote the extension of $\widehat{\mathcal{G}}_{A}^{(\Lambda)}$ by $\widehat{\mathbb{G}}_{m, A}$ by the 2 -cocycle

$$
C(X, Y)=F_{p}(c, \Lambda ; X, Y) \in Z^{2}\left(\widehat{\mathcal{G}}_{A}^{(\Lambda)}, \widehat{\mathbb{G}}_{m, A}\right) .
$$

Then a homomorphism of formal groups $\widehat{W}_{A} \rightarrow \mathcal{E}$ is given by $T_{0} \mapsto F_{0}(\boldsymbol{T})$, $T_{1} \mapsto F_{1}(\boldsymbol{T})$, where $F_{0}(\boldsymbol{T})=F_{0}\left(T_{0}, T_{1}, T_{2}, \ldots\right)$ and $F_{1}(\boldsymbol{T})=F_{1}\left(T_{0}, T_{1}, T_{2}, \ldots\right) \in$ $A\left[\left[T_{0}, T_{1}, T_{2}, \ldots\right]\right]$ satisfy the functional equations

$$
\begin{aligned}
& F_{0}(\boldsymbol{X}+\boldsymbol{Y})=F_{0}(\boldsymbol{X})+F_{0}(\boldsymbol{Y})+\Lambda F_{0}(\boldsymbol{X}) F_{0}(\boldsymbol{Y}) \\
& F_{1}(\boldsymbol{X}+\boldsymbol{Y})=F_{1}(\boldsymbol{X}) F_{1}(\boldsymbol{Y}) C\left(F_{0}(\boldsymbol{X}), F_{0}(\boldsymbol{Y})\right)
\end{aligned}
$$

We now define formal power series $\tilde{G}_{p}^{(\Lambda)}(\boldsymbol{Z}, \boldsymbol{U} ; \boldsymbol{T})$ and $G_{p}^{(\Lambda)}(\boldsymbol{Z}, \boldsymbol{U} ; \boldsymbol{T})$ to describe explicitly homomorphisms of $\widehat{W}_{A}$ to $\mathcal{E}$. First we establish a formalism for the extensions of $\widehat{\mathcal{G}}_{A}^{(\Lambda)}$ by $\widehat{\mathbb{G}}_{m, A}$.

4.29. Let

$$
\boldsymbol{U}=\left(U_{0}, U_{1}, U_{2}, \ldots\right), \quad \boldsymbol{Z}=\left(Z_{0}, Z_{1}, Z_{2}, \ldots\right)
$$

and

$$
\boldsymbol{T}=\left(T_{0}, T_{1}, T_{2}, \ldots\right)
$$

We define formal power series

$\tilde{G}_{p}^{(\Lambda)}(\boldsymbol{Z}, \boldsymbol{U} ; \boldsymbol{T}), G_{p}^{(\Lambda)}(\boldsymbol{Z}, \boldsymbol{U} ; \boldsymbol{T}) \in \mathbb{Q}\left[\Lambda, U_{0}, U_{1}, U_{2}, \ldots, Z_{0}, Z_{1}, Z_{2}, \ldots\right]\left[\left[T_{0}, T_{1}, T_{2}, \ldots\right]\right]$

by

$$
\tilde{G}_{p}^{(\Lambda)}(\boldsymbol{Z}, \boldsymbol{U} ; \boldsymbol{T})=\exp \left[\sum_{r=1}^{\infty}\left(\sum_{k=0}^{r-1} \frac{\Phi_{r}\left(\Lambda U_{0}, \ldots, \Lambda U_{k}, 0, \ldots, 0\right) \Phi_{r-k-1}(\boldsymbol{Z})}{\Lambda^{p^{r-k}}}\right) \frac{\Phi_{r}(\boldsymbol{T})}{p^{r}}\right]
$$

and

$$
G_{p}^{(\Lambda)}(\boldsymbol{Z}, \boldsymbol{U} ; \boldsymbol{T})=\frac{\tilde{G}_{p}^{(\Lambda)}(\boldsymbol{Z}, \boldsymbol{U} ; \boldsymbol{T})}{\prod_{r=0}^{\infty}\left[1+\Lambda^{p^{r+1}} E_{p}^{(\Lambda)}(\boldsymbol{U} ; \boldsymbol{T})^{p^{r+1}}\right]^{\frac{\Phi_{r}(\boldsymbol{Z})}{p^{r+1} \Lambda^{p^{r+1}}}}}
$$

It is readily seen that

$$
\tilde{G}_{p}^{(\Lambda)}(\boldsymbol{Z}, \boldsymbol{U} ; S(\boldsymbol{X}, \boldsymbol{Y}))=\tilde{G}_{p}^{(\Lambda)}(\boldsymbol{Z}, \boldsymbol{U} ; \boldsymbol{X}) \tilde{G}_{p}^{(\Lambda)}(\boldsymbol{Z}, \boldsymbol{U} ; \boldsymbol{Y})
$$

for $\boldsymbol{X}=\left(X_{0}, X_{1}, X_{2}, \ldots\right), \boldsymbol{Y}=\left(Y_{0}, Y_{1}, Y_{2}, \ldots\right)$. Here

$$
S(\boldsymbol{X}, \boldsymbol{Y})=\left(S_{0}(\boldsymbol{X}, \boldsymbol{Y}), S_{1}(\boldsymbol{X}, \boldsymbol{Y}), S_{2}(\boldsymbol{X}, \boldsymbol{Y}), \ldots\right)
$$


Moreover, we have

$$
\begin{aligned}
& \tilde{G}_{p}^{(\Lambda)}(\boldsymbol{Z}, \boldsymbol{U} ; \boldsymbol{T}) \\
& =\exp \left[\sum_{r=0}^{\infty} \frac{\Phi_{r}(\boldsymbol{Z})}{p^{r+1} \Lambda^{p^{r+1}}}\left(\sum_{k=0}^{\infty} \frac{\Phi_{r+k+1}\left(\Lambda U_{0}, \ldots, \Lambda U_{k}, 0, \ldots, 0\right) \Phi_{r+k+1}(\boldsymbol{T})}{p^{k}}\right)\right] .
\end{aligned}
$$

It follows that

$$
\tilde{G}_{p}^{(\Lambda)}(S(\boldsymbol{X}, \boldsymbol{Y}), \boldsymbol{U} ; \boldsymbol{T})=\tilde{G}_{p}^{(\Lambda)}(\boldsymbol{X}, \boldsymbol{U} ; \boldsymbol{T}) \tilde{G}_{p}^{(\Lambda)}(\boldsymbol{Y}, \boldsymbol{U} ; \boldsymbol{T})
$$

and

$$
G_{p}^{(\Lambda)}(S(\boldsymbol{X}, \boldsymbol{Y}), \boldsymbol{U} ; \boldsymbol{T})=G_{p}^{(\Lambda)}(\boldsymbol{X}, \boldsymbol{U} ; \boldsymbol{T}) G_{p}^{(\Lambda)}(\boldsymbol{Y}, \boldsymbol{U} ; \boldsymbol{T})
$$

for $\boldsymbol{X}=\left(X_{0}, X_{1}, X_{2}, \ldots\right), \boldsymbol{Y}=\left(Y_{0}, Y_{1}, Y_{2}, \ldots\right)$.

Proposition 4.30. Under the notation above, we have

$$
G_{p}^{(\Lambda)}(\boldsymbol{Z}, \boldsymbol{U} ; \boldsymbol{T}) \in \mathbb{Z}_{(p)}\left[\Lambda, U_{0}, U_{1}, U_{2}, \ldots, Z_{0}, Z_{1}, Z_{2}, \ldots\right]\left[\left[T_{0}, T_{1}, T_{2}, \ldots\right]\right]
$$

Proof. Put

$$
\left(\tilde{F}_{0}(\boldsymbol{W}), \tilde{F}_{1}(\boldsymbol{W}), \tilde{F}_{2}(\boldsymbol{W}), \ldots\right)=\left(F-\left[\Lambda^{p-1}\right]\right) \boldsymbol{W} \in W\left(\mathbb{Z}\left[\Lambda, W_{0}, W_{1}, W_{2}, \ldots\right]\right)
$$

and

$$
\begin{aligned}
& A=\mathbb{Z}_{(p)}\left[\Lambda, U_{0}, U_{1}, U_{2}, \ldots, Z_{0}, Z_{1}, Z_{2}, \ldots\right] \\
& B=A\left[W_{0}, W_{1}, W_{2}, \ldots\right] /\left(\tilde{F}_{0}(\boldsymbol{W})-Z_{0}, \tilde{F}_{1}(\boldsymbol{W})-Z_{1}, \tilde{F}_{2}(\boldsymbol{W})-Z_{2}, \ldots\right) .
\end{aligned}
$$

Then $B$ is flat over $A$ since $F-\left[\Lambda^{p-1}\right]: W_{\mathbb{Z}[\Lambda]} \rightarrow W_{\mathbb{Z}[\Lambda]}$ is faithfully flat ([13, Cor. 1.8]). Moreover,

$$
B \cap \mathbb{Q}\left[\Lambda, U_{0}, U_{1}, U_{2}, \ldots, Z_{0}, Z_{1}, Z_{2}, \ldots\right]=A \text {. }
$$

Then it is sufficient to prove that $G_{p}^{(\Lambda)}(\boldsymbol{Z}, \boldsymbol{U} ; \boldsymbol{T}) \in B\left[\left[T_{0}, T_{1}, T_{2}, \ldots\right]\right]$. We can verify this assertion thanks to the following two equalities:

$$
\begin{aligned}
& E_{p}\left(\boldsymbol{W}, \Lambda ; E_{p}^{(\Lambda)}(\boldsymbol{U}, \boldsymbol{T})\right) \\
& \quad=\left[1+\Lambda E_{p}^{(\Lambda)}(\boldsymbol{U} ; \boldsymbol{T})\right]^{\frac{\Phi_{0}(\boldsymbol{W})}{\Lambda}} \prod_{r=0}^{\infty}\left[1+\Lambda^{p^{r+1}} E_{p}^{(\Lambda)}(\boldsymbol{U} ; \boldsymbol{T})^{p^{r+1}}\right]^{\frac{\Phi_{r}(\boldsymbol{Z})}{p^{r+1} \Lambda^{p^{r+1}}}}
\end{aligned}
$$


and

$$
E_{p}\left(\sum_{k=0}^{\infty} V^{k}\left(\left[U_{k}\right] \boldsymbol{W}\right) ; \boldsymbol{T}\right)=\left[1+\Lambda E_{p}^{(\Lambda)}(\boldsymbol{U} ; \boldsymbol{T})\right]^{\frac{\Phi_{0}(\boldsymbol{W})}{\Lambda}} \tilde{G}_{p}^{(\Lambda)}(\boldsymbol{Z}, \boldsymbol{U} ; \boldsymbol{T})
$$

The first equality is a direct consequence of the definition:

$$
E_{p}(\boldsymbol{W}, \Lambda ; T)=(1+\Lambda T)^{\frac{\Phi_{0}(\boldsymbol{W})}{\Lambda}} \prod_{r=0}^{\infty}\left(1+\Lambda^{p^{r+1}} T^{p^{r+1}}\right) \frac{\Phi_{r+1}(\boldsymbol{W})-\Lambda^{p^{r}(p-1)} \Phi_{r}(\boldsymbol{W})}{p^{r+1} \Lambda^{p^{r+1}}}
$$

since we have $\boldsymbol{Z}=\left(F-\left[\Lambda^{p-1}\right]\right) \boldsymbol{W}$ and therefore $\Phi_{r}(\boldsymbol{Z})=\Phi_{r+1}(\boldsymbol{W})-$ $\Lambda^{p^{r}(p-1)} \Phi_{r}(\boldsymbol{W})$ for each $r \geq 0$.

Now we prove the second equality. By the definition, we have

$$
\left[1+\Lambda E_{p}^{(\Lambda)}(\boldsymbol{U} ; \boldsymbol{T})\right]^{\frac{\Phi_{0}(\boldsymbol{W})}{\Lambda}}=\exp \left[\frac{\Phi_{0}(\boldsymbol{W})}{\Lambda}\left(\sum_{r=0}^{\infty} \frac{\Phi_{r}\left(\Lambda U_{0}, \ldots, \Lambda U_{r}\right) \Phi_{r}(\boldsymbol{T})}{p^{r}}\right)\right]
$$

and

$$
\begin{aligned}
& \log \tilde{G}_{p}^{(\Lambda)}(\boldsymbol{Z}, \boldsymbol{U} ; \boldsymbol{T})=\sum_{r=1}^{\infty}\left(\sum_{k=0}^{r-1} \frac{\Phi_{r}\left(\Lambda U_{0}, \ldots, \Lambda U_{k}, 0, \ldots, 0\right) \Phi_{r-k-1}(\boldsymbol{Z})}{\Lambda^{p^{r-k}}}\right) \frac{\Phi_{r}(\boldsymbol{T})}{p^{r}} \\
& =\sum_{r=1}^{\infty}\left\{\sum_{k=0}^{r-1} \frac{\Phi_{r}\left(\Lambda U_{0}, \ldots, \Lambda U_{k}, 0, \ldots, 0\right)\left(\Phi_{r-k}(\boldsymbol{W})-\Lambda^{p^{r-k-1}(p-1)} \Phi_{r-k-1}(\boldsymbol{W})\right)}{\Lambda^{p^{r-k}}}\right\} \frac{\Phi_{r}(\boldsymbol{T})}{p^{r}}
\end{aligned}
$$

Hence the coefficient of $\frac{\Phi_{r}(\boldsymbol{T})}{p^{r}}$ in

$$
\log \tilde{G}_{p}^{(\Lambda)}(\boldsymbol{Z}, \boldsymbol{U} ; \boldsymbol{T})+\frac{\Phi_{0}(\boldsymbol{W})}{\Lambda}\left(\sum_{r=0}^{\infty} \frac{\Phi_{r}\left(\Lambda U_{0}, \ldots, \Lambda U_{r}\right) \Phi_{r}(\boldsymbol{T})}{p^{r}}\right)
$$

is given by

$$
\begin{aligned}
& \frac{\Phi_{r}\left(\Lambda U_{0}, 0, \ldots, 0\right)}{\Lambda^{p^{r}}} \Phi_{r}(\boldsymbol{W}) \\
& +\sum_{k=1}^{r} \frac{\left(\Phi_{r}\left(\Lambda U_{0}, \ldots, \Lambda U_{k}, 0, \ldots, 0\right)-\Phi_{r}\left(\Lambda U_{0}, \ldots, \Lambda U_{k-1}, 0, \ldots, 0\right)\right)}{\Lambda^{p^{r-k}}} \Phi_{r-k}(\boldsymbol{W}) .
\end{aligned}
$$

Noting that

$$
\begin{gathered}
\Phi_{r}\left(\Lambda U_{0}, \ldots, \Lambda U_{k-1}, \Lambda U_{k}, 0, \ldots, 0\right)-\Phi_{r}\left(\Lambda U_{0}, \ldots, \Lambda U_{k-1}, 0,0, \ldots, 0\right) \\
=p^{k}\left(\Lambda U_{k}\right)^{p^{r-k}}
\end{gathered}
$$


we obtain

$$
\begin{array}{r}
\log \left[1+\Lambda E_{p}^{(\Lambda)}(\boldsymbol{U} ; \boldsymbol{T})\right]^{\frac{\Phi_{0}(\boldsymbol{W})}{\Lambda}}+\log \tilde{G}_{p}^{(\Lambda)}(\boldsymbol{Z}, \boldsymbol{U} ; \boldsymbol{T}) \\
=\sum_{r=0}^{\infty}\left(\sum_{k=0}^{r} p^{k} U_{k}^{p^{r-k}} \Phi_{r-k}(\boldsymbol{W})\right) \frac{\Phi_{r}(\boldsymbol{T})}{p^{r}}
\end{array}
$$

This implies the result since we have

$$
\Phi_{r}\left(\sum_{k=0}^{\infty} V^{k}\left(\left[U_{k}\right] \boldsymbol{W}\right)\right)=\sum_{k=0}^{r} p^{k} U_{k}^{p^{r-k}} \Phi_{r-k}(\boldsymbol{W})
$$

for each $r \geq 0$.

EXAMPle 4.31. Specializing $\Lambda$ to 0 , we obtain

$$
\begin{aligned}
& G^{(0)}(\boldsymbol{Z}, \boldsymbol{U} ; \boldsymbol{T}) \\
& =\exp \left[\sum_{r=1}^{\infty}\left(\sum_{k=0}^{r-1} \frac{U_{k}^{p^{r-k}} \Phi_{r-k-1}(\boldsymbol{Z})}{p^{r-k}}\right) \Phi_{r}(\boldsymbol{T})-\sum_{k=0}^{\infty} \frac{\Phi_{k}(\boldsymbol{Z})}{p^{k+1}}\left(\sum_{r=0}^{\infty} U_{r} \Phi_{r}(\boldsymbol{T})\right)^{p^{k+1}}\right]
\end{aligned}
$$

Indeed, we have

$$
\begin{aligned}
\log \tilde{G}^{(\Lambda)}(\boldsymbol{Z}, \boldsymbol{U} ; \boldsymbol{T}) & =\sum_{r=1}^{\infty}\left(\sum_{k=0}^{r-1} \frac{\Phi_{r}\left(\Lambda U_{0}, \ldots, \Lambda U_{k}, 0, \ldots, 0\right) \Phi_{r-k-1}(\boldsymbol{Z})}{\Lambda^{p^{r-k}}}\right) \frac{\Phi_{r}(\boldsymbol{T})}{p^{r}} \\
& \equiv \sum_{r=1}^{\infty}\left(\sum_{k=0}^{r-1} \frac{U_{k}^{p^{r-k}} \Phi_{r-k-1}(\boldsymbol{Z})}{p^{r-k}}\right) \Phi_{r}(\boldsymbol{T}) \bmod \Lambda^{p}
\end{aligned}
$$

since

$$
\Phi_{r}\left(\Lambda U_{0}, \ldots, \Lambda U_{k}, 0, \ldots, 0\right)=\left(\Lambda U_{0}\right)^{p^{r}}+\cdots+p^{k-1}\left(\Lambda U_{k-1}\right)^{p^{r-k+1}}+p^{k}\left(\Lambda U_{k}\right)^{p^{r-k}}
$$

On the other hand, we have

$$
\begin{aligned}
\log [ & \left.\prod_{k=0}^{\infty}\left\{1+\Lambda^{p^{k+1}} E_{p}^{(\Lambda)}(\boldsymbol{U} ; \boldsymbol{T})^{p^{k+1}}\right\}^{\frac{\Phi_{k}(\boldsymbol{Z})}{p^{k+1} \Lambda^{p^{k+1}}}}\right] \\
& =\sum_{k=0}^{\infty} \frac{\Phi_{k}(\boldsymbol{Z})}{p^{k+1} \Lambda^{p^{k+1}}} \log \left[1+\Lambda^{p^{k+1}} E_{p}^{(\Lambda)}(\boldsymbol{U} ; \boldsymbol{T})^{p^{k+1}}\right] \\
& \equiv \sum_{k=0}^{\infty} \frac{\Phi_{k}(\boldsymbol{V})}{p^{k+1}} E_{p}^{(\Lambda)}(\boldsymbol{U} ; \boldsymbol{T})^{p^{k+1}} \equiv \sum_{k=0}^{\infty} \frac{\Phi_{r}(\boldsymbol{V})}{p^{r+1}}\left(\sum_{r=0}^{\infty} U_{r} \Phi_{r}(\boldsymbol{T})\right)^{p^{k+1}} \bmod \Lambda
\end{aligned}
$$


since

$$
E_{p}^{(\Lambda)}(\boldsymbol{U} ; \boldsymbol{T}) \equiv \sum_{r=0}^{\infty} U_{r} \Phi_{r}(\boldsymbol{T}) \bmod \Lambda
$$

as is remarked in Example 4.17.2. Combining the two congruence relations, we obtain

$$
\begin{aligned}
& G^{(\Lambda)}(\boldsymbol{Z}, \boldsymbol{U} ; \boldsymbol{T}) \\
& \equiv \exp \left[\sum_{r=1}^{\infty}\left(\sum_{k=1}^{r} \frac{U_{r-k}^{p^{k}} \Phi_{k-1}(\boldsymbol{Z})}{p^{k}}\right) \Phi_{r}(\boldsymbol{T})-\sum_{k=0}^{\infty} \frac{\Phi_{k}(\boldsymbol{Z})}{p^{k+1}}\left(\sum_{r=0}^{\infty} U_{r} \Phi_{r}(\boldsymbol{T})\right)^{p^{k+1}}\right] \bmod \Lambda
\end{aligned}
$$

Definition 4.32. Let $A$ be a $\mathbb{Z}_{(p)}[\Lambda]$-algebra, and let $\boldsymbol{a} \in W^{(\Lambda)}(A)$ and $\boldsymbol{c} \in W(A)$. We define a formal power series $G_{p}^{(\Lambda)}(\boldsymbol{c}, \boldsymbol{a} ; \boldsymbol{T}) \in A\left[\left[T_{0}, T_{1}, T_{2}, \ldots\right]\right]$, substituting $\boldsymbol{Z}=\boldsymbol{c}$ and $\boldsymbol{U}=\boldsymbol{a}$ in $G_{p}^{(\Lambda)}(\boldsymbol{Z}, \boldsymbol{U} ; \boldsymbol{T})$.

By the preceding argument on $G_{p}^{(\Lambda)}(\boldsymbol{Z}, \boldsymbol{U} ; \boldsymbol{T})$, we have

(1) $G_{p}^{(\Lambda)}\left(\boldsymbol{c}+\boldsymbol{c}^{\prime}, \boldsymbol{a} ; \boldsymbol{T}\right)=G_{p}^{(\Lambda)}(\boldsymbol{c}, \boldsymbol{a} ; \boldsymbol{T}) G_{p}^{(\Lambda)}\left(\boldsymbol{c}^{\prime}, \boldsymbol{a} ; \boldsymbol{T}\right)$;

(2) $G_{p}^{(\Lambda)}\left(\left(F-\left[\Lambda^{p-1}\right]\right) \boldsymbol{c}, \boldsymbol{a} ; \boldsymbol{T}\right)=\frac{E_{p}\left(\sum_{k=0}^{\infty} V^{k}\left(\left[a_{k}\right] \boldsymbol{c}\right) ; \boldsymbol{T}\right)}{E_{p}\left(\boldsymbol{c}, \Lambda ; E_{p}^{(\Lambda)}(\boldsymbol{a} ; \boldsymbol{T})\right)}$.

Lemma 4.33. Let $A$ be $a \mathbb{Z}_{(p)}[\Lambda]$-algebra and $\boldsymbol{a} \in W^{(\Lambda)}(A), \boldsymbol{b}, \boldsymbol{c} \in W(A)$. Let $\mathcal{E}$ denote the extension of $\widehat{\mathcal{G}}_{A}^{(\Lambda)}$ by $\widehat{\mathbb{G}}_{m, A}$ defined by the 2 -cocycle $F_{p}(\boldsymbol{c}, \Lambda ; X, Y) \in$ $Z^{2}\left(\widehat{\mathcal{G}}_{A}^{(\Lambda)}, \widehat{\mathbb{G}}_{m, A}\right)$. Then

$$
\begin{aligned}
T_{0} \mapsto E_{p}^{(\Lambda)}(\boldsymbol{a} ; \boldsymbol{T}), T_{1} \mapsto E_{p}(\boldsymbol{b} ; \boldsymbol{T}) G_{p}^{(\Lambda)}(\boldsymbol{c}, \boldsymbol{a} ; \boldsymbol{T}): \\
\widehat{W}_{A}=\operatorname{Spf} A\left[\left[T_{0}, T_{1}, T_{2}, \ldots\right]\right] \longrightarrow \mathcal{E}=\operatorname{Spf} A\left[\left[T_{0}, T_{1}-1\right]\right]
\end{aligned}
$$

defines a homomorphism of formal groups $\widehat{W}_{A} \rightarrow \mathcal{E}$.

Proof. By Remark 4.28.1, the assertion is equivalent to the equalities:

$$
E_{p}^{(\Lambda)}(\boldsymbol{a} ; S(\boldsymbol{X}, \boldsymbol{Y}))=E_{p}^{(\Lambda)}(\boldsymbol{a} ; \boldsymbol{X})+E_{p}^{(\Lambda)}(\boldsymbol{a} ; \boldsymbol{Y})+\Lambda E_{p}^{(\Lambda)}(\boldsymbol{a} ; \boldsymbol{X}) E_{p}^{(\Lambda)}(\boldsymbol{a} ; \boldsymbol{Y})
$$

and

$$
\begin{aligned}
E_{p}(\boldsymbol{b} ; S(\boldsymbol{X}, \boldsymbol{Y})) G_{p}^{(\Lambda)}(\boldsymbol{c}, \boldsymbol{a} ; S(\boldsymbol{X}, \boldsymbol{Y}))=\left\{E_{p}(\boldsymbol{b} ; \boldsymbol{X}) G_{p}^{(\Lambda)}(\boldsymbol{c}, \boldsymbol{a} ; \boldsymbol{X})\right\} \\
\left\{E_{p}(\boldsymbol{b} ; \boldsymbol{Y}) G_{p}^{(\Lambda)}(\boldsymbol{c}, \boldsymbol{a} ; \boldsymbol{Y})\right\} F_{p}\left(\boldsymbol{c}, \Lambda ; E_{p}^{(\Lambda)}(\boldsymbol{a} ; \boldsymbol{X}), E_{p}^{(\Lambda)}(\boldsymbol{a} ; \boldsymbol{Y})\right) .
\end{aligned}
$$

Here

$$
S(\boldsymbol{X}, \boldsymbol{Y})=\left(S_{0}(\boldsymbol{X}, \boldsymbol{Y}), S_{1}(\boldsymbol{X}, \boldsymbol{Y}), S_{2}(\boldsymbol{X}, \boldsymbol{Y}), \ldots\right)
$$


Then it is sufficient to verify the equality

$$
\frac{G_{p}^{(\Lambda)}(\boldsymbol{c}, \boldsymbol{a} ; S(\boldsymbol{X}, \boldsymbol{Y}))}{G_{p}^{(\Lambda)}(\boldsymbol{c}, \boldsymbol{a} ; \boldsymbol{X}) G_{p}^{(\Lambda)}(\boldsymbol{c}, \boldsymbol{a} ; \boldsymbol{Y})}=F_{p}\left(\boldsymbol{c}, \Lambda ; E_{p}^{(\Lambda)}(\boldsymbol{a} ; \boldsymbol{X}), E_{p}^{(\Lambda)}(\boldsymbol{a} ; \boldsymbol{Y})\right)
$$

by $4.18,(2)$ and $4.14,(2)$.

We may assume that

$$
A=\mathbb{Z}_{(p)}\left[\Lambda, U_{0}, U_{1}, U_{2}, \ldots, Z_{0}, Z_{1}, Z_{2}, \ldots\right]
$$

and

$$
\boldsymbol{a}=\boldsymbol{U}=\left(U_{0}, U_{1}, U_{2}, \ldots\right), \quad \boldsymbol{c}=\boldsymbol{Z}=\left(Z_{0}, Z_{1}, Z_{2}, \ldots\right) .
$$

First we have

$$
\begin{gathered}
\prod_{r=0}^{\infty}\left[\frac{\left(1+\Lambda^{p^{r+1}} E_{p}^{(\Lambda)}(\boldsymbol{U} ; \boldsymbol{X})^{p^{r+1}}\right)\left(1+\Lambda^{p^{r+1}} E_{p}^{(\Lambda)}(\boldsymbol{U} ; \boldsymbol{Y})^{p^{r+1}}\right)}{1+\Lambda^{p^{r+1}} E_{p}^{(\Lambda)}(\boldsymbol{U} ; S(\boldsymbol{X}, \boldsymbol{Y}))^{p^{r+1}}}\right]^{\frac{\Phi_{r}(\boldsymbol{z})}{p^{r+1} \Lambda^{p^{r+1}}}} \\
=F_{p}\left(\boldsymbol{Z}, \Lambda ; E_{p}^{(\Lambda)}(\boldsymbol{U} ; \boldsymbol{X}), E_{p}^{(\Lambda)}(\boldsymbol{U} ; \boldsymbol{Y})\right)
\end{gathered}
$$

combining the definition

$$
F_{p}(\boldsymbol{Z}, \Lambda ; X, Y)=\prod_{r=0}^{\infty}\left[\frac{\left(1+\Lambda^{p^{r+1}} X^{p^{r+1}}\right)\left(1+\Lambda^{p^{r+1}} Y^{p^{r+1}}\right)}{1+\Lambda^{p^{r+1}}(X+Y+\Lambda X Y)^{p^{r+1}}}\right]^{\frac{\Phi_{r}(\boldsymbol{Z})}{p^{r+1} \Lambda^{r+1}}}
$$

and the equality

$$
E_{p}^{(\Lambda)}(\boldsymbol{U} ; S(\boldsymbol{X}, \boldsymbol{Y}))=E_{p}^{(\Lambda)}(\boldsymbol{U} ; \boldsymbol{X})+E_{p}^{(\Lambda)}(\boldsymbol{U} ; \boldsymbol{Y})+\Lambda E_{p}^{(\Lambda)}(\boldsymbol{U} ; \boldsymbol{X}) E_{p}^{(\Lambda)}(\boldsymbol{U} ; \boldsymbol{Y}) .
$$

On the other hand, we have

$$
\tilde{G}^{(\Lambda)}(\boldsymbol{Z}, \boldsymbol{U} ; \boldsymbol{X}) \tilde{G}^{(\Lambda)}(\boldsymbol{Z}, \boldsymbol{U} ; \boldsymbol{Y})=\tilde{G}^{(\Lambda)}(\boldsymbol{Z}, \boldsymbol{U} ; S(\boldsymbol{X}, \boldsymbol{Y})) .
$$

Hence we obtain the required equality.

Proposition 4.34. Let $A$ be a $\mathbb{Z}_{(p)}[\Lambda]$-algebra and $\boldsymbol{c} \in W(A)$. Let $\mathcal{E}$ denote the extension of $\widehat{\mathcal{G}}_{A}^{(\Lambda)}$ by $\widehat{\mathbb{G}}_{m, A}$ defined by the 2-cocycle $F_{p}(\boldsymbol{c}, \Lambda ; X, Y) \in$ $Z^{2}\left(\widehat{\mathcal{G}}_{A}^{(\Lambda)}, \widehat{\mathbb{G}}_{m, A}\right)$, and let $E$ denote the extension of $W^{(\Lambda)}(A)$ by $W(A)$ defined by the class $c$ in

$$
\operatorname{Coker}\left[F-\left[\Lambda^{p-1}\right]: W(A) \rightarrow W(A)\right]=\operatorname{Ext}_{D_{A}}^{1}\left(W^{(\Lambda)}(A), W(A)\right) .
$$

Then

$$
(\boldsymbol{a}, \boldsymbol{b}) \mapsto\left(\left(T_{0}, T_{1}\right) \mapsto\left(E_{p}^{(\Lambda)}(\boldsymbol{a} ; \boldsymbol{T}), E_{p}(\boldsymbol{b} ; \boldsymbol{T}) G_{p}^{(\Lambda)}(\boldsymbol{c}, \boldsymbol{a} ; \boldsymbol{T})\right)\right)
$$


gives rise to an isomorphism $\eta: E \rightarrow C(\mathcal{E})=\operatorname{Hom}_{A-\mathrm{gr}}\left(\widehat{W}_{A}, \mathcal{E}\right)$.

Proof. Let $\tilde{\boldsymbol{c}}$ be the 2-cocycle of $Z^{2}\left(W^{(\Lambda)}(A), W(A)\right)$ defined by $\boldsymbol{c}$ as in Remark 4.10. We begin with verifying that $\eta$ is a homomorphism. By Remark 4.10, this is equivalent to the equalities:

$$
E_{p}^{(\Lambda)}\left(\boldsymbol{a}+{ }^{(\Lambda)} \boldsymbol{a}^{\prime} ; \boldsymbol{T}\right)=E_{p}^{(\Lambda)}(\boldsymbol{a} ; \boldsymbol{T})+E_{p}^{(\Lambda)}\left(\boldsymbol{a}^{\prime} ; \boldsymbol{T}\right)+\Lambda E_{p}^{(\Lambda)}(\boldsymbol{a} ; \boldsymbol{T}) E_{p}^{(\Lambda)}\left(\boldsymbol{a}^{\prime} ; \boldsymbol{T}\right)
$$

and

$$
\begin{aligned}
E_{p}\left(\boldsymbol{b}+\boldsymbol{b}^{\prime}+\tilde{\boldsymbol{c}}\left(\boldsymbol{a}, \boldsymbol{a}^{\prime}\right)\right. & ; \boldsymbol{T}) G_{p}^{(\Lambda)}\left(\boldsymbol{c}, \boldsymbol{a}+{ }^{(\Lambda)} \boldsymbol{a}^{\prime} ; \boldsymbol{T}\right)=\left\{E_{p}(\boldsymbol{b} ; \boldsymbol{T}) G_{p}^{(\Lambda)}(\boldsymbol{c}, \boldsymbol{a} ; \boldsymbol{T})\right\} \\
& \left\{E_{p}\left(\boldsymbol{b}^{\prime} ; \boldsymbol{Y}\right) G_{p}^{(\Lambda)}\left(\boldsymbol{c}, \boldsymbol{a}^{\prime} ; \boldsymbol{T}\right)\right\} F_{p}\left(\boldsymbol{c}, \Lambda ; E_{p}^{(\Lambda)}(\boldsymbol{a} ; \boldsymbol{T}), E_{p}^{(\Lambda)}\left(\boldsymbol{a}^{\prime} ; \boldsymbol{T}\right)\right) .
\end{aligned}
$$

Then it is sufficient to verify the equality

$$
\frac{G_{p}^{(\Lambda)}(\boldsymbol{c}, \boldsymbol{a} ; \boldsymbol{T}) G_{p}^{(\Lambda)}\left(\boldsymbol{c}, \boldsymbol{a}^{\prime} ; \boldsymbol{T}\right)}{G_{p}^{(\Lambda)}\left(\boldsymbol{c}, \boldsymbol{a}+{ }^{(\Lambda)} \boldsymbol{a}^{\prime} ; \boldsymbol{T}\right)}=\frac{E_{p}\left(\tilde{\boldsymbol{c}}\left(\boldsymbol{a}, \boldsymbol{a}^{\prime}\right) ; \boldsymbol{T}\right)}{F_{p}\left(\boldsymbol{c}, \Lambda ; E_{p}^{(\Lambda)}(\boldsymbol{a} ; \boldsymbol{T}), E_{p}^{(\Lambda)}\left(\boldsymbol{a}^{\prime} ; \boldsymbol{T}\right)\right)}
$$

by $4.18,(1)$ and $4.14,(1)$.

We may assume that

$$
A=\mathbb{Z}_{(p)}\left[\Lambda, U_{0}, U_{1}, U_{2}, \ldots, U_{0}^{\prime}, U_{1}^{\prime}, U_{2}^{\prime}, \ldots, Z_{0}, Z_{1}, Z_{2}, \ldots\right]
$$

and

$$
\boldsymbol{a}=\boldsymbol{U}=\left(U_{0}, U_{1}, U_{2}, \ldots\right), \boldsymbol{a}^{\prime}=\boldsymbol{U}^{\prime}=\left(U_{0}^{\prime}, U_{1}^{\prime}, U_{2}^{\prime}, \ldots\right), \boldsymbol{c}=\boldsymbol{Z}=\left(Z_{0}, Z_{1}, Z_{2}, \ldots\right) .
$$

First we have

$$
\begin{aligned}
& \prod_{k=0}^{\infty}\left[\frac{\left(1+\Lambda^{p^{k+1}} E_{p}^{(\Lambda)}(\boldsymbol{U} ; \boldsymbol{T})^{p^{k+1}}\right)\left(1+\Lambda^{p^{k+1}} E_{p}^{(\Lambda)}(\boldsymbol{U} ; \boldsymbol{T})^{p^{k+1}}\right)}{1+\Lambda^{p^{k+1}} E_{p}^{(\Lambda)}\left(\boldsymbol{U}+(\Lambda) \boldsymbol{U}^{\prime} ; \boldsymbol{T}\right)^{p^{k+1}}}\right]^{\frac{\boldsymbol{\Phi}_{k}(\boldsymbol{Z})}{p^{k+1} \Lambda^{p^{k+1}}}} \\
& =F_{p}\left(\boldsymbol{Z}, \Lambda ; E_{p}^{(\Lambda)}(\boldsymbol{U} ; \boldsymbol{T}), E_{p}^{(\Lambda)}\left(\boldsymbol{U}^{\prime} ; \boldsymbol{T}\right)\right)
\end{aligned}
$$

combining the definition

$$
F_{p}(Z, \Lambda ; X, Y)=\prod_{r=0}^{\infty}\left[\frac{\left(1+\Lambda^{p^{r+1}} X^{p^{r+1}}\right)\left(1+\Lambda^{p^{r+1}} Y^{p^{r+1}}\right)}{1+\Lambda^{p^{r+1}}(X+Y+\Lambda X Y)^{p^{r+1}}}\right]^{\frac{\Phi_{r}(\boldsymbol{Z})}{p^{r+1} \Lambda^{p^{r+1}}}}
$$

and the equalities

$$
E_{p}^{(\Lambda)}\left(\boldsymbol{U}+{ }^{(\Lambda)} \boldsymbol{U}^{\prime} ; \boldsymbol{T}\right)=E_{p}^{(\Lambda)}(\boldsymbol{U} ; \boldsymbol{T})+E_{p}^{(\Lambda)}\left(\boldsymbol{U}^{\prime} ; \boldsymbol{T}\right)+\Lambda E_{p}^{(\Lambda)}(\boldsymbol{U} ; \boldsymbol{T}) E_{p}^{(\Lambda)}\left(\boldsymbol{U}^{\prime} ; \boldsymbol{T}\right)
$$


(Proposition 4.18,(1)). On the other hand, by the definition,

$$
\tilde{G}_{p}^{(\Lambda)}(\boldsymbol{Z}, \boldsymbol{U} ; \boldsymbol{T})=\exp \left[\sum_{r=1}^{\infty}\left(\sum_{k=0}^{r-1} \frac{\Phi_{r}\left(\Lambda U_{0}, \ldots, \Lambda U_{k}, 0, \ldots, 0\right) \Phi_{r-k-1}(\boldsymbol{Z})}{\Lambda^{p^{r-k}}}\right) \frac{\Phi_{r}(\boldsymbol{T})}{p^{r}}\right]
$$

Moreover, as is shown in 4.11 , we have

$$
\begin{array}{r}
\Phi_{r}\left(\tilde{c}\left(\boldsymbol{U}, \boldsymbol{U}^{\prime}\right)\right)=\left\{\sum_{k=0}^{r-1} \Phi_{r}\left(\Lambda U_{0}, \ldots, \Lambda U_{k}, 0, \ldots, 0\right)+\Phi_{r}\left(\Lambda U_{0}^{\prime}, \ldots, \Lambda U_{k}^{\prime}, 0, \ldots, 0\right)\right. \\
\left.-\Phi_{r}\left(\Lambda U_{0}^{\prime \prime}, \ldots, \Lambda U_{k}^{\prime \prime}, 0, \ldots, 0\right)\right\} \frac{\Phi_{r-k-1}(\boldsymbol{Z})}{\Lambda^{p^{r-k}}}
\end{array}
$$

if we put $\boldsymbol{U}+{ }^{(\Lambda)} \boldsymbol{U}^{\prime}=\left(U_{0}^{\prime \prime}, U_{1}^{\prime \prime}, U_{2}^{\prime \prime}, \ldots\right)$. Hence we obtain

$$
\frac{\tilde{G}_{p}^{(\Lambda)}(\boldsymbol{Z}, \boldsymbol{U} ; \boldsymbol{T}) \tilde{G}_{p}^{(\Lambda)}\left(\boldsymbol{Z}, \boldsymbol{U}^{\prime} ; \boldsymbol{T}\right)}{\tilde{G}_{p}^{(\Lambda)}\left(\boldsymbol{Z}, \boldsymbol{U}^{\prime \prime} ; \boldsymbol{T}\right)}=E_{p}\left(\tilde{c}\left(\boldsymbol{U}, \boldsymbol{U}^{\prime}\right) ; \boldsymbol{T}\right)
$$

and the required equality.

Now we verify the bijectivity of $\eta: E \rightarrow \operatorname{Hom}_{A-\mathrm{gr}}\left(\widehat{W}_{A}, \mathcal{E}\right)$. Consider the commutative diagram with exact rows

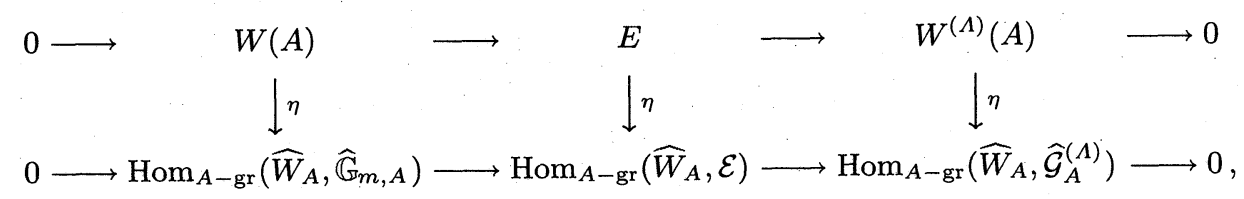

where $\eta: W(A) \rightarrow \operatorname{Hom}_{A-\mathrm{gr}}\left(\widehat{W}_{A}, \widehat{\mathbb{G}}_{m, A}\right)$ and $\eta: W^{(\Lambda)}(A) \rightarrow \operatorname{Hom}_{A-\mathrm{gr}}\left(\widehat{W}_{A}, \widehat{\mathcal{G}}_{A}^{(\Lambda)}\right)$ are defined by $\boldsymbol{b} \mapsto E_{p}(\boldsymbol{b} ; \boldsymbol{T})$ and $\boldsymbol{a} \mapsto E_{p}^{(\Lambda)}(\boldsymbol{a} ; \boldsymbol{T})$ respectively, and the row below is induced from the exact sequence of the formal groups

$$
0 \rightarrow \widehat{\mathbb{G}}_{m, A} \rightarrow \mathcal{E} \rightarrow \widehat{\mathcal{G}}_{A}^{(\Lambda)} \rightarrow 0 .
$$

We can conclude that the middle vertical arrow is bijective since the two sided arrows are bijective (Example 4.14 and Proposition 4.19).

Now we establish a formalism for the extensions of $\widehat{\mathcal{G}}_{A}^{(\Lambda)}$ by $\widehat{\mathcal{G}}_{A}^{(M)}$, paraphrasing the preceding argument.

4.35. Let

$$
\boldsymbol{U}=\left(U_{0}, U_{1}, U_{2}, \ldots\right), \quad \boldsymbol{V}=\left(V_{0}, V_{1}, V_{2}, \ldots\right), \quad \boldsymbol{Z}=\left(U_{0}, U_{1}, U_{2}, \ldots\right)
$$

and

$$
\boldsymbol{T}=\left(T_{0}, T_{1}, T_{2}, \ldots\right)
$$


We define a formal power series

$$
\begin{aligned}
& E_{p}^{(\Lambda, M)}(\boldsymbol{Z}, \boldsymbol{U}, \boldsymbol{V} ; \boldsymbol{T}) \\
& \quad \in \mathbb{Z}_{(p)}\left[\Lambda, M, U_{0}, U_{1}, U_{2}, \ldots, V_{0}, V_{1}, V_{2}, \ldots, Z_{0}, Z_{1}, Z_{2}, \ldots\right]\left[\left[T_{0}, T_{1}, T_{2}, \ldots\right]\right]
\end{aligned}
$$

by

$$
E_{p}^{(\Lambda, M)}(\boldsymbol{Z}, \boldsymbol{U}, \boldsymbol{V} ; \boldsymbol{T})=\frac{1}{M}\left[E_{p}\left(\alpha^{(M)} \boldsymbol{V} ; \boldsymbol{T}\right) G_{p}^{(\Lambda)}\left(\alpha^{(M)} \boldsymbol{Z}, \boldsymbol{U} ; \boldsymbol{T}\right)-1\right]
$$

EXAmPle 4.36 . Specializing $\Lambda$ and $M$ to 0 , we obtain

$$
\begin{aligned}
& E^{(0,0)}(\boldsymbol{Z}, \boldsymbol{U}, \boldsymbol{V} ; \boldsymbol{T}) \\
& =\sum_{r=0}^{\infty} V_{r} \Phi_{r}(\boldsymbol{Z})+\frac{1}{p}\left[\sum_{r=1}^{\infty}\left(\sum_{k=0}^{r-1} U_{k}^{p^{r-k}} Z_{r-k-1}\right) \Phi_{r}(\boldsymbol{T})-\sum_{k=0}^{\infty} Z_{k}\left(\sum_{r=0}^{\infty} U_{r} \Phi_{r}(\boldsymbol{T})\right)^{p^{k+1}}\right] .
\end{aligned}
$$

Indeed, we have

$$
E_{p}\left(\alpha^{(M)} \boldsymbol{V} ; \boldsymbol{T}\right)=\exp \left[\sum_{r=0}^{\infty} \frac{\Phi_{r}\left(\alpha^{(M)} \boldsymbol{V}\right) \Phi_{r}(\boldsymbol{T})}{p^{r}}\right] \equiv 1+M\left[\sum_{r=0}^{\infty} V_{r} \Phi_{r}(\boldsymbol{T})\right] \bmod M^{2}
$$

since

$$
\Phi_{r}\left(\alpha^{(M)} \boldsymbol{V}\right)=\Phi_{r}\left(M V_{0}, \ldots, M V_{r}\right) \equiv p^{r} M V_{r} \bmod M^{p}
$$

for each $r \geq 0$. On the other hand, we have

$$
\begin{array}{r}
G^{(\Lambda)}\left(\alpha^{(M)} \boldsymbol{Z}, \boldsymbol{U} ; \boldsymbol{T}\right) \\
\equiv 1+\frac{M}{p}\left[\sum_{r=1}^{\infty}\left(\sum_{k=0}^{r-1} U_{k}^{p^{r-k}} Z_{r-k-1}\right) \Phi_{r}(\boldsymbol{T})-\sum_{k=0}^{\infty} Z_{k}\left(\sum_{r=0}^{\infty} U_{r} \Phi_{r}(\boldsymbol{T})\right)^{p^{k+1}}\right] \\
\bmod \left(\Lambda, M^{2}\right)
\end{array}
$$

since

$$
\begin{aligned}
G^{(\Lambda)}\left(\alpha^{(M)} \boldsymbol{Z}, \boldsymbol{U} ; \boldsymbol{T}\right) \\
\equiv \exp \left[\sum_{r=1}^{\infty}\left(\sum_{k=0}^{r-1} \frac{U_{k}^{p^{r-k}} \Phi_{r-k-1}\left(\alpha^{(M)} \boldsymbol{Z}\right)}{p^{r-k}}\right) \Phi_{r}(\boldsymbol{T})\right. \\
\left.\quad-\sum_{k=0}^{\infty} \frac{\Phi_{k}\left(\alpha^{(M)} \mathbb{Z}\right)}{p^{k+1}}\left(\sum_{r=0}^{\infty} U_{r} \Phi_{r}(\boldsymbol{T})\right)^{p^{k+1}}\right] \bmod \Lambda
\end{aligned}
$$

and

$$
\Phi_{k}\left(\alpha^{(M)} Z\right)=\Phi_{k}\left(M Z_{0}, \ldots, M Z_{k}\right) \equiv p^{k} M Z_{k} \quad \bmod M^{p}
$$


for each $k \geq 0$. Combining the two congruence relations, we obtain

$$
\begin{aligned}
& E^{(\Lambda, M)}(\boldsymbol{Z}, \boldsymbol{U}, \boldsymbol{V} ; \boldsymbol{T}) \\
& =\frac{1}{M}\left[E_{p}\left(\alpha^{(M)} \boldsymbol{V}, \boldsymbol{T}\right) G_{p}^{(\Lambda)}\left(\alpha^{(M)} \boldsymbol{Z}, \boldsymbol{U} ; \boldsymbol{T}\right)-1\right] \equiv \sum_{r=0}^{\infty} V_{r} \Phi_{r}(\boldsymbol{Z}) \\
& +\frac{1}{p}\left[\sum_{r=1}^{\infty}\left(\sum_{k=0}^{r-1} U_{k}^{p^{r-k}} Z_{r-k-1}\right) \Phi_{r}(\boldsymbol{T})-\sum_{k=0}^{\infty} Z_{k}\left(\sum_{r=0}^{\infty} U_{r} \Phi_{r}(\boldsymbol{T})\right)^{p^{k+1}}\right] \bmod (\Lambda, M) .
\end{aligned}
$$

Definition 4.37. Let $A$ be a $\mathbb{Z}_{(p)}[\Lambda, M]$-algebra, and let $\boldsymbol{a} \in W^{(\Lambda)}(A)$ and $\boldsymbol{b}, \boldsymbol{c} \in W^{(M)}(A)$. We define a formal power series $E_{p}^{(\Lambda, M)}(\boldsymbol{c}, \boldsymbol{a}, \boldsymbol{b} ; \boldsymbol{T}) \in$ $A\left[\left[T_{0}, T_{1}, T_{2}, \ldots\right]\right]$, substituting $\boldsymbol{U}=\boldsymbol{a}, \boldsymbol{V}=\boldsymbol{b}$ and $\boldsymbol{Z}=\boldsymbol{c}$ in $E_{p}^{(\Lambda, M)}(\boldsymbol{Z}, \boldsymbol{U}, \boldsymbol{V} ; \boldsymbol{T})$.

As in 4.32, we see

$$
\begin{aligned}
E_{p}^{(\Lambda, M)}\left(\boldsymbol{c}+{ }^{(M)} \boldsymbol{c}^{\prime}, \boldsymbol{a}, \boldsymbol{b}+{ }^{(M)} \boldsymbol{b}^{\prime} ; \boldsymbol{T}\right)=E_{p}^{(\Lambda)}(\boldsymbol{c}, \boldsymbol{a}, \boldsymbol{b} ; \boldsymbol{T}) \\
+E_{p}^{(\Lambda, M)}\left(\boldsymbol{c}^{\prime}, \boldsymbol{a}, \boldsymbol{b}^{\prime} ; \boldsymbol{T}\right)+M E_{p}^{(\Lambda, M)}(\boldsymbol{c}, \boldsymbol{a}, \boldsymbol{b} ; \boldsymbol{T}) E_{p}^{(\Lambda, M)}\left(\boldsymbol{c}^{\prime}, \boldsymbol{a}, \boldsymbol{b}^{\prime} ; \boldsymbol{T}\right)
\end{aligned}
$$

(2) $E_{p}^{(\Lambda, M)}\left(\left(F^{(M)}-\left[\Lambda^{p-1}\right]\right) \boldsymbol{c}, \boldsymbol{a}, \boldsymbol{b} ; \boldsymbol{T}\right)$

$$
=\frac{E_{p}^{(M)}\left(\boldsymbol{b}+{ }^{(M)} \sum_{k=0}^{\infty} V^{k}\left(\left[a_{k}\right] \boldsymbol{c}\right) ; \boldsymbol{T}\right)-E_{p}^{(M)}\left(\boldsymbol{c}, \Lambda ; E_{p}^{(\Lambda)}(\boldsymbol{a} ; \boldsymbol{T})\right)}{1+M E_{p}^{(M)}\left(\boldsymbol{c}, \Lambda ; E_{p}^{(\Lambda)}(\boldsymbol{a} ; \boldsymbol{T})\right)} .
$$

Lemma 4.38. Let $A$ be a $\mathbb{Z}_{(p)}[\Lambda, M]$-algebra and $\boldsymbol{a} \in W^{(\Lambda)}(A), \boldsymbol{b}, \boldsymbol{c} \in$ $W^{(M)}(A)$. Let $\mathcal{E}$ denote the extension of $\widehat{\mathcal{G}}_{A}^{(\Lambda)}$ by $\widehat{\mathcal{G}}_{A}^{(M)}$ defined by the 2-cocycle $F_{p}^{(M)}(\boldsymbol{c}, \Lambda ; X, Y) \in Z^{2}\left(\widehat{\mathcal{G}}_{A}^{(\Lambda)}, \widehat{\mathcal{G}}_{A}^{(M)}\right)$. Then

$$
\begin{aligned}
T_{0} \mapsto E_{p}^{(\Lambda)}(\boldsymbol{a} ; \boldsymbol{T}), T_{1} \mapsto E_{p}^{(\Lambda, M)}(\boldsymbol{c}, \boldsymbol{a}, \boldsymbol{b} ; \boldsymbol{T}): \\
\widehat{W}_{A}=\operatorname{Spf} A\left[\left[T_{0}, T_{1}, T_{2}, \ldots\right]\right] \longrightarrow \mathcal{E}=\operatorname{Spf} A\left[\left[T_{0}, T_{1}\right]\right]
\end{aligned}
$$

defines a homomorphism of formal groups $\widehat{W}_{A} \rightarrow \mathcal{E}$.

Proof. We may assume that

$$
A=\mathbb{Z}_{(p)}\left[\Lambda, M, U_{0}, U_{1}, U_{2}, \ldots, V_{0}, V_{1}, V_{2}, \ldots, Z_{0}, Z_{1}, Z_{2}, \ldots\right]
$$

and

$$
\boldsymbol{a}=\boldsymbol{U}=\left(U_{0}, U_{1}, U_{2}, \ldots\right), \quad \boldsymbol{b}=\boldsymbol{V}=\left(V_{0}, V_{1}, V_{2}, \ldots\right), \boldsymbol{c}=\boldsymbol{Z}=\left(Z_{0}, Z_{1}, Z_{2}, \ldots\right) .
$$

Put

$$
F_{0}(\boldsymbol{T})=E_{p}^{(\Lambda)}(\boldsymbol{U} ; \boldsymbol{T}), \quad F_{1}(\boldsymbol{T})=E_{p}^{(\Lambda, M)}(\boldsymbol{Z}, \boldsymbol{U}, \boldsymbol{V} ; \boldsymbol{T})
$$


and

$$
C(X, Y)=F_{p}^{(M)}(c, \Lambda ; X, Y)
$$

Then by Remark 4.28 , it is sufficient to verify that

$$
\begin{aligned}
F_{1}(\boldsymbol{X}+\boldsymbol{Y})=F_{1} & (\boldsymbol{X})+F_{1}(\boldsymbol{Y})+C\left(F_{0}(\boldsymbol{X}), F_{0}(\boldsymbol{Y})\right) \\
& +M\left\{F_{1}(\boldsymbol{X}) F_{1}(\boldsymbol{Y})+\left(F_{1}(\boldsymbol{X})+F_{1}(\boldsymbol{Y})\right) C\left(F_{0}(\boldsymbol{X}), F_{0}(\boldsymbol{Y})\right)\right\} \\
& +M^{2} F_{1}(\boldsymbol{X}) F_{1}(\boldsymbol{Y}) C\left(F_{0}(\boldsymbol{X}), F_{0}(\boldsymbol{Y})\right)
\end{aligned}
$$

which is a direct consequence of the equality proved in 4.33:

$$
1+M F_{1}(\boldsymbol{X}+\boldsymbol{Y})=\left\{1+M F_{1}(\boldsymbol{X})\right\}\left\{1+M F_{1}(\boldsymbol{Y})\right\}\left\{1+M C\left(F_{0}(\boldsymbol{X}), F_{0}(\boldsymbol{Y})\right)\right\}
$$

Proposition 4.39. Let $A$ be $a \mathbb{Z}_{(p)}[\Lambda, M]$-algebra and $\boldsymbol{c} \in W^{(M)}$. Let $\mathcal{E}$ denote the extension of $\widehat{\mathcal{G}}_{A}^{(\Lambda)}$ by $\widehat{\mathcal{G}}_{A}^{(M)}$ defined by the 2-cocycle $F_{p}^{(M)}(\boldsymbol{c}, \Lambda ; X, Y) \in$ $Z^{2}\left(\widehat{\mathcal{G}}_{A}^{(\Lambda)}, \widehat{\mathcal{G}}_{A}^{(M)}\right)$, and let $E$ denote the extension of $W^{(\Lambda)}(A)$ by $W^{(M)}(A)$ defined by the class $\boldsymbol{c}$ in

$$
\operatorname{Coker}\left[F^{(M)}-\left[\Lambda^{p-1}\right]: W^{(M)}(A) \rightarrow W^{(M)}(A)\right]=\operatorname{Ext}_{D_{A}}^{1}\left(W^{(\Lambda)}(A), W^{(M)}(A)\right) .
$$

Then

$$
(\boldsymbol{a}, \boldsymbol{b}) \mapsto\left[\left(T_{0}, T_{1}\right) \mapsto\left(E_{p}^{(\Lambda)}(\boldsymbol{a} ; \boldsymbol{T}), E_{p}^{(\Lambda, M)}(\boldsymbol{c}, \boldsymbol{a}, \boldsymbol{b} ; \boldsymbol{T})\right)\right]
$$

gives rise to an isomorphism $\eta: E \rightarrow C(\mathcal{E})=\operatorname{Hom}_{A-\mathrm{gr}}\left(\widehat{W}_{A}, \mathcal{E}\right)$.

Proof. We can deduce the assertion from Proposition 4.34 similarly as Lemma 4.38 is deduced from Lemma 4.33 , and thus omit the details.

At. last we attain the second main result on the compatibility of the isomorphism $\xi$ with the Cartier theory. phism

4.40. Let $A$ be a $\mathbb{Z}_{(p)}[\Lambda, M]$-algebra. By Theorem 3.5.1 we have an isomor-

$$
\xi: \operatorname{Coker}\left[F^{(M)}-\left[\Lambda^{p-1}\right]: W^{(M)}(A) \rightarrow W^{(M)}(A)\right] \stackrel{\sim}{\longrightarrow} H_{0}^{2}\left(\widehat{\mathcal{G}}_{A}^{(\Lambda)}, \widehat{\mathcal{G}}_{A}^{(M)}\right) .
$$

On the other hand, the functor $C: G \mapsto C(G)=\operatorname{Hom}_{A-\mathrm{gr}}\left(\widehat{W}_{A}, G\right)$ is exact since $C$ is fully faithful (the second thereom of the Cartier theory, cf. [4, Th. 27.7.10]) and $C\left(\widehat{W}_{A}\right)$ is isomorphic to $D_{A}$. Hence from a commutative extension of formal groups

$$
0 \rightarrow \widehat{\mathcal{G}}_{A}^{(M)} \rightarrow \mathcal{E} \rightarrow \widehat{\mathcal{G}}_{A}^{(\Lambda)} \rightarrow 0
$$

we obtain an extension of $D_{A}$-modules

$$
0 \rightarrow C\left(\widehat{\mathcal{G}}_{A}^{(M)}\right) \rightarrow C(\mathcal{E}) \rightarrow C\left(\widehat{\mathcal{G}}_{A}^{(\Lambda)}\right) \rightarrow 0
$$


The correspondence $\mathcal{E} \mapsto C(\mathcal{E})$ gives rise to an injective homomorphism

$$
\operatorname{Ext}_{A}^{1}\left(\widehat{\mathcal{G}}_{A}^{(\Lambda)}, \widehat{\mathcal{G}}_{A}^{(M)}\right) \longrightarrow \operatorname{Ext}_{D_{A}}^{1}\left(C\left(\widehat{\mathcal{G}}_{A}^{(\Lambda)}\right), C\left(\widehat{\mathcal{G}}_{A}^{(M)}\right)\right)
$$

since $G \mapsto C(G)$ is fully faithful. Note that $H_{0}^{2}\left(\widehat{\mathcal{G}}_{A}^{(\Lambda)}, \widehat{\mathcal{G}}_{A}^{(M)}\right)$ is the subgroup of $\operatorname{Ext}_{A}^{1}\left(\widehat{\mathcal{G}}_{A}^{(\Lambda)}, \widehat{\mathcal{G}}_{A}^{(M)}\right)$ formed by the class of extensions with a section.

Now we have isomorphisms

$$
\eta: W^{(\Lambda)}(A) \stackrel{\sim}{\longrightarrow} C\left(\widehat{\mathcal{G}}_{A}^{(\Lambda)}\right)
$$

and

$$
\eta: W^{(M)}(A) \stackrel{\sim}{\longrightarrow} C\left(\widehat{\mathcal{G}}_{A}^{(M)}\right)
$$

by Theorem 4.15.1. Furthermore, as is remarked in 4.9, we have an isomorphism

$$
\operatorname{Ext}_{D_{A}}^{1}\left(W^{(\Lambda)}(A), W^{(M)}(A)\right) \stackrel{\sim}{\longrightarrow} \operatorname{Coker}\left[F^{(M)}-\left[\Lambda^{p-1}\right]: W^{(M)}(A) \rightarrow W^{(M)}(A)\right],
$$

defined by $\partial \boldsymbol{a} \mapsto[\boldsymbol{a}]$. Here $\partial: W^{(M)}(A)=\operatorname{Hom}_{D_{A}}\left(D_{A}, W^{(M)}(A)\right) \rightarrow$ $\operatorname{Ext}_{D_{A}}^{1}\left(W^{(\Lambda)}(A), W^{(M)}(A)\right)$ is the boundary map induced by the exact sequence

$$
0 \rightarrow D_{A} \stackrel{\mathbb{F}-\left[\Lambda^{p-1}\right]}{\longrightarrow} D_{A} \rightarrow W^{(\Lambda)}(A) \rightarrow 0
$$

Theorem 4.40.1. Let $\boldsymbol{a} \in \operatorname{Coker}\left[F^{(M)}-\left[\Lambda^{p-1}\right]: W^{(M)}(A) \rightarrow W^{(M)}(A)\right]$. Then we have

$$
C(\xi(a))=a
$$

under the identification

$$
\operatorname{Ext}_{D_{A}}^{1}\left(C\left(\widehat{\mathcal{G}}_{A}^{(\Lambda)}\right), C\left(\widehat{\mathcal{G}}_{A}^{(M)}\right)\right)=\operatorname{Coker}\left[F^{(M)}-\left[\Lambda^{p-1}\right]: W^{(M)}(A) \rightarrow W^{(M)}(A)\right]
$$

Proof. Take $c \in W^{(M)}(A)$ representing $\boldsymbol{a}$. We define homomorphisms of formal groups

$$
\tilde{\varphi}_{\Lambda}: \widehat{W}_{A}=\operatorname{Spf} A\left[\left[T_{0}, T_{1}, T_{2}, \ldots\right]\right] \rightarrow \widehat{\mathcal{G}}_{A}^{(\Lambda)}=\operatorname{Spf} A[[T]]
$$

and

$$
\tilde{\varphi}_{(M, c)}: \widehat{W}_{A}=\operatorname{Spf} A\left[\left[T_{0}, T_{1}, T_{2}, \ldots\right]\right] \rightarrow \widehat{\mathcal{G}}_{A}^{(M)}=\operatorname{Spf} A[[T]]
$$

by

$$
T \mapsto E_{p}^{(\Lambda)}\left([1]_{\Lambda} ; T\right): A[[T]] \rightarrow A\left[\left[T_{0}, T_{1}, T_{2}, \ldots\right]\right]
$$

and

$$
T \mapsto E_{p}^{(M)}(\boldsymbol{c} ; \boldsymbol{T}): A[[T]] \rightarrow A\left[\left[T_{0}, T_{1}, T_{2}, \ldots\right]\right]
$$


respectively. It is readily seen that $C\left(\tilde{\varphi}_{\Lambda}\right): D_{A}=C\left(\widehat{W}_{A}\right) \rightarrow W^{(\Lambda)}(A)=C\left(\widehat{\mathcal{G}}_{A}^{(\Lambda)}\right)$ is given by $\varphi_{\Lambda}: 1 \mapsto[1]_{\Lambda}$, and $C\left(\tilde{\varphi}_{(M, c)}\right): D_{A}=C\left(\widehat{W}_{A}\right) \rightarrow W^{(M)}(A)=C\left(\widehat{\mathcal{G}}_{A}^{(M)}\right)$ is given by $\varphi_{(M, c)}: 1 \mapsto c$.

Let $\mathcal{E}$ denote the extension of $\widehat{\mathcal{G}}_{A}^{(\Lambda)}$ by $\widehat{\mathcal{G}}_{A}^{(M)}$ defined by the 2-cocycle $F_{p}^{(M)}(\boldsymbol{c}, \Lambda ; X, Y) \in Z^{2}\left(\widehat{\mathcal{G}}_{A}^{(\Lambda)}, \widehat{\mathcal{G}}_{A}^{(M)}\right)$. We define a homomorphism of formal groups

$$
\tilde{\varphi}: \widehat{W}_{A}=\operatorname{Spf} A\left[\left[T_{0}, T_{1}, T_{2}, \ldots\right]\right] \rightarrow \mathcal{E}=\operatorname{Spf} A\left[\left[T_{0}, T_{1}\right]\right]
$$

by

$$
\left(T_{0}, T_{1}\right) \mapsto\left(E_{p}^{(\Lambda)}\left([1]_{\Lambda} ; \boldsymbol{T}\right), E_{p}^{(\Lambda, M)}\left(\boldsymbol{c},[1]_{\Lambda},[0]_{M} ; \boldsymbol{T}\right)\right)
$$

(cf. Lemma 4.38). Note that $\varphi=C(\tilde{\varphi}): D_{A}=C\left(\widehat{W}_{A}\right) \rightarrow E=C(\mathcal{E})$ is given by $1 \mapsto\left([1]_{\Lambda},[0]_{M}\right)$ when $E$ is identified with the set $W^{(\Lambda)}(A) \times W^{(M)}(A)$ as in 4.10 (cf. Proposition 4.39).

Consider now a diagram of formal groups with exact rows:

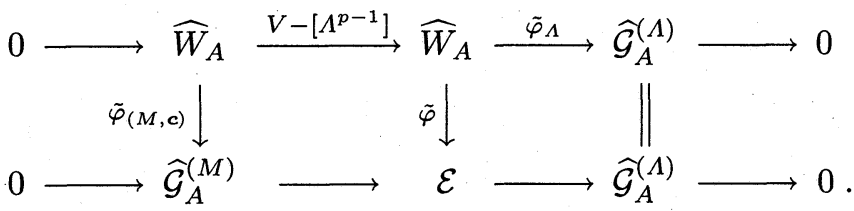

Then the corresponding diagram of the Cartier modules are isomorphic to the commutative diagram of left $D_{A}$-modules with exact rows:

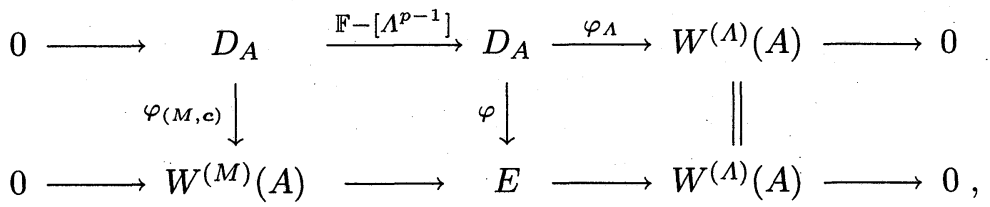

which appears in the proof of Proposition 4.9.1. More precisely, the left square is a push-down, and therefore, $E$ is the extension of $W^{(\Lambda)}(A)$ by $W^{(M)}(A)$ defined by the class $c$ in

$$
\operatorname{Coker}\left[F^{(M)}-\left[\Lambda^{p-1}\right]: W^{(M)}(A) \rightarrow W^{(M)}(A)\right]=\operatorname{Ext}_{D_{A}}^{1}\left(W^{(\Lambda)}(A), W^{(M)}(A)\right) .
$$

Thus we obtain the assertion of the theorem.

REMARK 4.41. We can deduce the commutativity of the first diagram above from the faithfulness of the Cartier functor and the commutativity of the second diagram above, and we obtain the following formula:

(1) Let $A$ be a $\mathbb{Z}_{(p)}[\Lambda, M]$-algebra and $c \in W^{(M)}(A)$.

$$
E_{p}^{(\Lambda, M)}\left(\boldsymbol{c},[1]_{\Lambda},[0]_{M} ;\left(V-\left[\Lambda^{p-1}\right]\right) \boldsymbol{T}\right)=E_{p}^{(M)}(\boldsymbol{c} ; \boldsymbol{T}) .
$$


In particular, we have:

(2) Let $A$ be a $\mathbb{Z}_{(p)}[\Lambda]$-algebra and $c \in W(A)$. Then we have

$$
G_{p}^{(\Lambda)}\left(c,[1]_{\Lambda} ;\left(V-\left[\Lambda^{p-1}\right]\right) \boldsymbol{T}\right)=E_{p}(\boldsymbol{c} ; \boldsymbol{T}) .
$$

We can verify the formula directly as follows.

To prove (2), we may assume that $A=\mathbb{Z}_{(p)}\left[Z_{0}, Z_{1}, Z_{2}, \ldots\right]$ and $\boldsymbol{c}=\boldsymbol{Z}=$ $\left(Z_{0}, Z_{1}, Z_{2}, \ldots\right)$. By the definition we have

$$
G_{p}^{(\Lambda)}\left(\boldsymbol{Z},[1]_{\Lambda} ; \boldsymbol{T}\right)=\frac{\tilde{G}_{p}^{(\Lambda)}\left(\boldsymbol{Z},[1]_{\Lambda} ; \boldsymbol{T}\right)}{\prod_{r=0}^{\infty}\left[1+\Lambda^{p^{r+1}} E_{p}^{(\Lambda)}\left([1]_{\Lambda} ; \boldsymbol{T}\right)^{p^{r+1}}\right] \frac{\Phi_{r}(\boldsymbol{Z})}{p^{r+1} \Lambda^{r+1}}} .
$$

First we obtain

$$
E_{p}^{(\Lambda)}\left([1]_{\Lambda} ;\left(V-\left[\Lambda^{p-1}\right]\right) T\right)=0
$$

since we have

$$
E_{p}^{(\Lambda)}\left([1]_{\Lambda} ;\left(\mathbb{V}-\left[\Lambda^{p-1}\right]\right) \boldsymbol{T}\right)=E_{p}^{(\Lambda)}\left(\left(F^{(\Lambda)}-\left[\Lambda^{p-1}\right]\right)[1]_{\Lambda} ; \boldsymbol{T}\right)
$$

by Proposition $4.18,(5)$ and

$$
\left(F^{(\Lambda)}-\left[\Lambda^{p-1}\right]\right)[1]_{\Lambda}=\left[\Lambda^{p-1}\right]-\left[\Lambda^{p-1}\right]=0
$$

by Lemma 1.13.1. On the other hand, we have

$$
\log \tilde{G}_{p}^{(\Lambda)}\left(\boldsymbol{Z},[1]_{\Lambda} ; \boldsymbol{T}\right)=\sum_{r=1}^{\infty}\left(\sum_{k=0}^{r-1} \frac{\Phi_{r}([\Lambda]) \Phi_{r-k-1}(\boldsymbol{Z})}{\Lambda^{p^{r-k}}}\right) \frac{\Phi_{r}(\boldsymbol{T})}{p^{r}}
$$

by the definition. Now we have

$$
\Phi_{r}\left(\left(V-\left[\Lambda^{p-1}\right]\right) \boldsymbol{T}\right)=p \Phi_{r-1}(\boldsymbol{T})-\Lambda^{p^{r}(p-1)} \Phi_{r}(\boldsymbol{T})
$$

for each $r>0$. It follows that

$$
\begin{aligned}
& \log \tilde{G}_{p}^{(\Lambda)}\left(\boldsymbol{W},[1]_{\Lambda} ;\left(V-\left[\Lambda^{p-1}\right]\right) \boldsymbol{T}\right) \\
&=\sum_{r=1}^{\infty}\left(\sum_{k=0}^{r-1} \frac{\Phi_{r}([\Lambda]) \Phi_{r-k-1}(\boldsymbol{Z})}{\Lambda^{p^{r-k}}}\right) \frac{\Phi_{r-1}(\boldsymbol{T})}{p^{r-1}} \\
&-\sum_{r=1}^{\infty}\left(\sum_{k=0}^{r-1} \frac{\Phi_{r}([\Lambda]) \Phi_{r-k-1}(\boldsymbol{Z})}{\Lambda^{p^{r-k}}}\right) \frac{\Lambda^{p^{r}(p-1)} \Phi_{r}(\boldsymbol{T})}{p^{r}}
\end{aligned}
$$




$$
\begin{aligned}
& =\sum_{r=1}^{\infty}\left(\sum_{k=0}^{r-1} \frac{\Lambda^{p^{r}} \Phi_{r-k-1}(\boldsymbol{Z})}{\Lambda^{p^{r-k}}}\right) \frac{\Phi_{r-1}(\boldsymbol{T})}{p^{r-1}} \\
& \quad-\sum_{r=1}^{\infty}\left(\sum_{k=0}^{r-1} \frac{\Lambda^{p^{r}} \Phi_{r-k-1}(\boldsymbol{Z})}{\Lambda^{p^{r-k}}}\right) \frac{\Lambda^{p^{r}(p-1)} \Phi_{r}(\boldsymbol{T})}{p^{r}} \\
& =\Phi_{0}(\boldsymbol{Z}) \Phi_{0}(\boldsymbol{T})+\sum_{r=1}^{\infty}\left(\sum_{k=0}^{r} \frac{\Lambda^{p^{r+1}} \Phi_{r-k}(\boldsymbol{Z})}{\Lambda^{p^{r-k+1}}}-\sum_{k=1}^{r} \frac{\Lambda^{p^{r+1}} \Phi_{r-k}(\boldsymbol{Z})}{\Lambda^{p^{r-k+1}}}\right) \frac{\Phi_{r}(\boldsymbol{T})}{p^{r}} \\
& =\sum_{r=0}^{\infty} \frac{\Phi_{r}(\boldsymbol{Z}) \Phi_{r}(\boldsymbol{T})}{p^{r}}=\log E_{p}(\boldsymbol{Z} ; \boldsymbol{T}) .
\end{aligned}
$$

Hence we obtain the required equality.

We can deduce (1) from (2) by the definition of $E_{p}^{(\Lambda, M)}(\boldsymbol{Z}, \boldsymbol{U}, \boldsymbol{V} ; \boldsymbol{T})$ since we have

$$
\alpha^{(M)}\left(\left(F^{(M)}-\left[\Lambda^{p-1}\right]\right) c\right)=\left(F-\left[\Lambda^{p-1}\right]\right) \alpha^{(M)}(\boldsymbol{c})
$$

by Proposition 1.16 .

REMARK 4.42. From the theorem, we can conclude the following assertion:

Let $A$ be a $\mathbb{Z}_{(p)}[\Lambda, M]$-algebra. Then we have

$$
H_{0}^{2}\left(\widehat{\mathcal{G}}_{A}^{(\Lambda)}, \widehat{\mathcal{G}}_{A}^{(M)}\right)=\operatorname{Ext}_{A}^{1}\left(\widehat{\mathcal{G}}_{A}^{(\Lambda)}, \widehat{\mathcal{G}}_{A}^{(M)}\right)
$$

EXAMPLE 4.43. The class of $\widehat{W}_{2}$ in $\operatorname{Ext}_{A}^{1}\left(\widehat{\mathbb{G}}_{a, A}, \widehat{\mathbb{G}}_{a, A}\right)$ is given by the class of $[1]_{0}$ under the identification

$$
H_{0}^{2}\left(\widehat{\mathbb{G}}_{a, A}, \widehat{\mathbb{G}}_{a, A}\right)=\operatorname{Coker}\left[F^{(0)}: W^{(0)}(A) \rightarrow W^{(0)}(A)\right],
$$

which follows from Remark 2.7.2. Moreover, we have

$$
E^{(0,0)}([1], \boldsymbol{U}, \boldsymbol{V} ; \boldsymbol{T})=\sum_{r=0}^{\infty} V_{r} \Phi_{r}(\boldsymbol{T})+\frac{1}{p}\left[\sum_{r=0}^{\infty} U_{r}^{p} \Phi_{r+1}(\boldsymbol{T})-\left(\sum_{r=0}^{\infty} U_{r} \Phi_{r}(\boldsymbol{T})\right)^{p}\right]
$$

as is remarked in Example 4.36. In particular, we obtain $E^{(0,0)}([1],[1],[0] ; \boldsymbol{T})=T_{1}$. Hence $([1],[0]) \in W^{(0)}(A) \times W^{(0)}(A)$ corresponds to the restriction map $R: \widehat{W}_{A} \rightarrow$ $\widehat{W}_{2, A}$ since we have $E^{(0)}([1] ; \boldsymbol{T})=T_{0}$.

\section{References}

[1] N. Bourbaki, Algèbre commutative, Chapitres 8 et 9, Masson, Paris, 1983.

[2] M. Demazure and P. Gabriel, Groupes algébriques, Tome 1, Masson-North-Holland, ParisAmsterdam, 1970.

[3] B. Green and M. Matignon, Liftings of Galois covers of smooth curves, Compositio Math., 113 (1998), 237-272. 
[4] M. Hazewinkel, Formal groups and applications, Academic Press, New York, 1978.

[5] L. Illusie, Complexe de de Rham-Witt et cohomologie cristalline, Ann. Sci. École Norm. Sup., (4) 12 (1979), 501-661.

[6 ] T. Sekiguchi, On the deformations of Witt groups to tori II, J. Algebra, 138 (1991), 273-297.

[ 7 ] T. Sekiguchi and N. Suwa, A case of extensions of group schemes over a discrete valuation ring, Tsukuba J. Math., 14 (1990), 459-487.

[8] T. Sekiguchi and N. Suwa, Some cases of extensions of group schemes over a discrete valuation ring I, J. Fac. Sci. Univ. Tokyo, 38 (1991), 1-45.

[9] T. Sekiguchi and N. Suwa, A note on extensions of algebraic and formal groups I, Math. Z., 206 (1991), 567-575.

[10] T. Sekiguchi and N. Suwa, A note on extensions of algebraic and formal groups II, Math. Z., 217 (1994), 447-457.

[11] T. Sekiguchi and N. Suwa, Théories de Kummer-Artin-Schreier-Witt, C. R. Acad. Sci. Paris, 319 (1994), 105-110.

[12] T. Sekiguchi and N. Suwa, A note on extensions of algebraic and formal groups III, Tôhoku Math. J., 49 (1997), 241-257.

[13] T. Sekiguchi and N. Suwa, A note on extensions of algebraic and formal groups IV, Tôhoku Math. J., 53 (2001), 203-240.

[14] T. Sekiguchi and N. Suwa, On the unified Kummer-Artin-Schreier-Witt theory, Prépublication No.111, Université de Bordeaux (1999).

[15] T. Sekiguchi, F. Oort and N. Suwa, On the deformation of Artin-Schreier to Kummer, Ann. Sci. École Norm. Sup., (4) 22 (1989), 345-375.

[16] M. Yato, On the extensions of $\widehat{\mathcal{G}}^{(\lambda)}$ by $\widehat{\mathbb{G}}_{a}$ over a $\mathbb{Z}_{(p)}$-algebra, Tokyo J. Math., 25 (2002), 107-137.

TSUTOMU SeKiguchi

Department of Mathematics

Chuo UnIVERSITY

1-13-27 KASUGA, BUNKYO-KU

TOKYO 112-8551, JAPAN

E-mail: sekiguti@math.chuo-u.ac.jp

\author{
NORIYUKI SuWA \\ Department of Mathematics \\ Chuo University \\ 1-13-27 Kasuga, Bunkyo-KU \\ TOKYO 112-8551, JAPAN \\ E-mail: suwa@math.chuo-u.ac.jp
}

